\title{
Elektrische Anisotropie durch ausgerichtete Olivinkristalle im oberen \\ Mantel in Mitteleuropa: \\ Magnetotellurische Array-Messungen und ein Ansatz zum Vergleich mit seismischer Anisotropie
}

\author{
Dissertation \\ zur Erlangung des Doktorgrades \\ der Mathematisch-Naturwissenschaftlichen Fakultäten \\ der Georg-August-Universität zu Göttingen \\ vorgelegt von \\ Alexander Gatzemeier \\ aus Northeim
}

Göttingen 2001 
D7

Referent:

Prof. Dr. Karsten Bahr

Korreferent:

Prof. Dr. Andreas Tilgner

Tag der mündlichen Prüfung: 25.01.2002 
Phantasie ist wichtiger als Wissen, denn Wissen ist begrenzt.

Albert Einstein 



\section{Inhaltsverzeichnis}

$\begin{array}{ll}\text { Einleitung } & 11\end{array}$

1 Grundlagen 14

1.1 Induktion in der Erde . . . . . . . . . . . . . . . . . . . . . . . . . . . . . . .

1.2 Magnetotellurik . . . . . . . . . . . . . . . . . 15

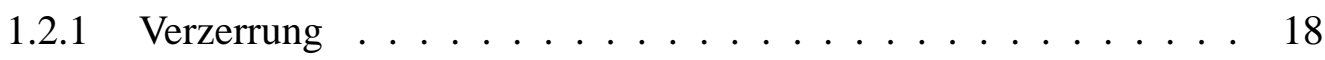

1.2.2 Strukturbestimmung . . . . . . . . . . . . . 20

1.3 Erdmagnetische Tiefensondierung . . . . . . . . . . . . . . 21

1.4 Anisotropie der Leitfähigkeit . . . . . . . . . . . . . . . . . . . 22

2 Elektromagnetische Arraymessungen $\quad 24$

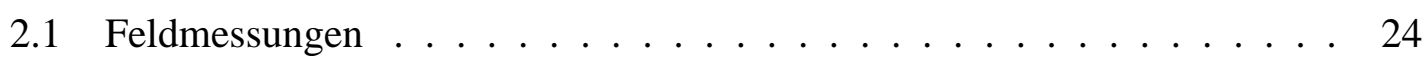

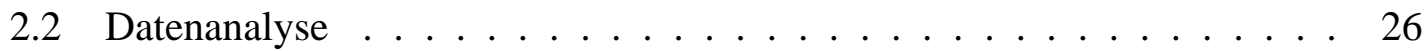

2.3 Ergebnisse der Datenanalyse . . . . . . . . . . . . . . . . . . 27

2.3.1 Die Phase der Impedanz . . . . . . . . . . . . . . . . 29

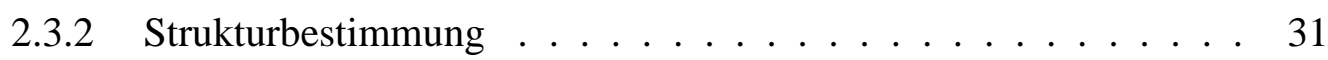

2.3.3 Lokale Induktionspfeile . . . . . . . . . . . . . 37

$3 \quad$ Ein Modell der Leitfähigkeitsverteilung $\quad 39$

3.1 Ein einfaches Leitfähigkeitsmodell für das Messgebiet . . . . . . . . . . 40

3.1.1 Vorüberlegungen: 1D-Modell . . . . . . . . . . . . . . 40

3.1.2 3D-Leitfähigkeitsmodell . . . . . . . . . . . . . . . . . . 41

3.2 Modellergebnisse und Datenanpassung . . . . . . . . . . . . . . 47

3.2.1 Die Phase der Impedanz . . . . . . . . . . . . . . . . . . . . . . . . . . . . . . . . . . . . . 49

3.2.2 Strukturanpassung . . . . . . . . . . . . . . . . . . . . . . . . . . . . . . 49

3.3 Einfluss krustaler Strukturen _. . . . . . . . . . . . . . . . 54

3.4 Zusammenfassung: Ein anisotroper oberer Mantel? . . . . . . . . . . 55

4 Leitfähigkeit und Anisotropie $\quad 57$

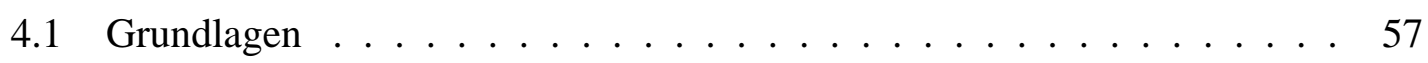

4.1.1 Leitfähigkeitsmechanismen ............. 57 
4.1.2 Effektive Leitfähigkeit von Gesteinen . . . . . . . . . . . . . . . 60

4.2 Leitfähigkeiten im oberen Mantel . . . . . . . . . . . . . . . . . 61

4.2.1 Trockener Olivin . . . . . . . . . . . . . . . . . . . 62

4.2.2 Partielle Schmelze . . . . . . . . . . . . . . . 65

4.2 .3 Wasser im oberen Mantel . . . . . . . . . . . . . . . . . 69

4.3 Anisotropie der elektrischen Leitfähigkeit . . . . . . . . . . . . . . . 72

5 Gemeinsame Interpretation seismischer und elektrischer Anisotropie $\quad 76$

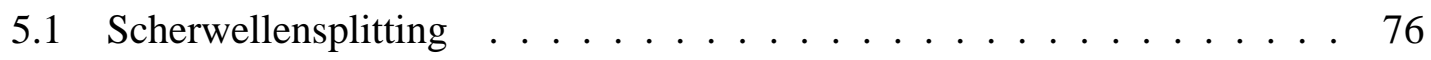

5.1 .1 Theorie ...................... 76

5.1.2 Ergebnisse im Messgebiet . . . . . . . . . . . . . 78

5.2 Interpretation seismischer und elektrischer Anisotropie . . . . . . . . . 81

6 Schlussfolgerungen $\quad 85$

$\begin{array}{lr}\text { A Anhang } & 89\end{array}$

A.1 Informationen zu den Messstationen . . . . . . . . . . . . . . . 89

A.2 Verwendete Programme . . . . . . . . . . . . . . . . . 91

A.2.1 Datenprozessing und Auswertung . . . . . . . . . . . . 91

A.2.2 3D Vorwärtsmodellierung: $\mathbf{m t d 3 f w d}$ und d3_to_mt . . . . . . . 92

A.3 Mischungsgesetze . . . . . . . . . . . . . . . . . . . . 95 


\section{Abbildungsverzeichnis}

1.1 Abschnittsweise Überlagerung richtungsabhängiger Leitfähigkeiten. . . . 21

1.2 Modellvorstellung makro- und mikroskopischer Anisotropie. . . . . . . . 23

2.1 Karte des Messgebietes mit den 64 MT-Stationen. . . . . . . . . . . . . 25

2.2 Karte des Messgebietes mit acht charakteristischen Stationen. . . . . . . . 27

2.3 Unrotierte Phasenkurven der acht charakteristischen Stationen. . . . . . . 28

2.4 Flächenhafte Darstellung der unrotierten Phasen bei 85 s. . . . . . . . . . 29

2.5 Flächenhafte Darstellung der unrotierten Phasen bei 2731 s. . . . . . . . . 30

2.6 Frequenzgang des phasensensitiven Rotationswinkels der 8 Stationen. . . 32

2.7 Phasensensitive Rotationswinkel der 8 Stationen. . . . . . . . . . . . . 33

2.8 Rotationswinkel und rotierte Phasen bei 85 und 2731 s. . . . . . . . . . . 34

2.9 Differenz der rotierten Phasen beider Polarisationen bei 2731 s. . . . . . . 35

2.10 Phasen des rotierten Impedanztensors der Station PANO. . . . . . . . . . 36

$2.11 \rho^{*}$-z*-Darstellung zweier Stationen im nördlichen Teil des Arrays. . . . . 37

2.12 Flächenhafte Darstellung der lokale Induktionspfeile bei 2731 s. . . . . . 38

3.1 1D-Anpassung der unrotierten Daten der Station ARN. . . . . . . . . . . 41

3.2 Das Modellgrid. . . . . . . . . . . . . . . . . . . . . 42

3.3 Vertikalschnitt durch das 1D-Hintergrundmodell. . . . . . . . . . . . 43

3.4 Das Leitfähigkeitsmodell in der oberen bis mittleren Kruste. . . . . . . . 44

3.5 Horizontalschnitt der Modellschicht der mittleren bis unteren Kruste. . . . 45

3.6 Horizontalschnitt der Modellschicht des oberen Mantels. . . . . . . . . . 46

3.7 Unrotierte Phasenkurven der acht Stationen mit Modellantwort. . . . . . . 48

3.8 Modellantwort: Flächenhafte Darstellung der Phasen bei 2731 s. . . . . . 49

3.9 Modellantwort des Rotationswinkels der acht Stationen. . . . . . . . . . . 50

3.10 Gemeinsame Darstellung der Drehwinkel von Modell und Daten. . . . . . 51

3.11 Drehwinkel und Phasendifferenz der Modellantwort. . . . . . . . . . . . 52

$3.12 \rho^{*}-z^{*}$-Darstellung der Modellantwort der Stationen ARN und MEDE. . . 53

3.13 Vergleich der Phasen für das Modell mit und ohne oberer Kruste. . . . . . 54

4.1 Energiezustände der Elektronen im Festkörper . . . . . . . . . . . . . . . 58

4.2 Die Hauptachsen des Olivinkristalls . . . . . . . . . . . . . . . . 62 
4.3 Leitfähigkeit von Olivin: SO2-Modell . . . . . . . . . . . . . . . . 63

4.4 Effektive Leitfähigkeiten aus Mischungsgesetzen . . . . . . . . . . . . . 64

4.5 Soliduskurven von Lherzolithen unter verschiedenen Mantelbedingungen. 66

4.6 Leitfähigkeiten partieller Schmelze . . . . . . . . . . . . . . . . . . . 67

4.7 Maximale Leitfähigkeiten partieller Schmelze . . . . . . . . . . . . . . . . 68

4.8 Leitfähigkeiten durch Diffusion von $\mathrm{H}^{+}$in Olivin . . . . . . . . . . . . 71

4.9 Vergleich der Leitfähigkeitsmechanismen . . . . . . . . . . . . . . 73

4.10 Diffusionskonstanten für Wasserstoff in Olivin . . . . . . . . . . . . . . . 74

5.1 Ausbreitung von Scherwellen in anisotropem Medium. . . . . . . . . . 77

5.2 Ausbreitungsweg der SKS-Phase schematisch dargestellt. . . . . . . . . . 78

5.3 Scherwellensplitting im Bereich des Messgebietes . . . . . . . . . . . . . 79

5.4 Abhängigkeit der Richtung $\phi$ vom Backazimut $\gamma$. . . . . . . . . . . . 80

5.5 Gemeinsame Interpretation seismischer und elektrischer Anisotropie . . . 83 


\section{Tabellenverzeichnis}

2.1 Für die Analyse festgelegte Auswertefrequenzen. . . . . . . . . . . . . . 26

4.1 Aktivierungsenthalpien gesteinsbildender Minerale . . . . . . . . . . . 63

$4.2 \mathrm{H}^{+}$-Sättigungskonzentrationen . . . . . . . . . . . . . . 70

5.1 Modellparameter des anisotropen 2-Schicht-Modells. . . . . . . . . . . . 80

5.2 Elektrische und seismische Anisotropie. . . . . . . . . . . . . . 82

6.1 Übersicht der Leitfähigkeitsmechanismen. . . . . . . . . . . . . . . 86

A.1 Tabelle der im Rahmen dieser Arbeit vermessenen Stationen. . . . . . . . 89

A.2 Tabelle der im Rahmen vorheriger Arbeiten vermessenen Stationen. . . . 89

A.3 Listing der Erweiterung des Hilfsprogramms d3_to_mt. . . . . . . . . . 92 


\section{Einleitung}

Für den Bereich der mittleren und unteren Kruste zeigen mehrere Untersuchungen eine Zone erhöhter Leitfähigkeit (HAAK und HUTTON 1986; JONES 1992). Obwohl die Ursache der hohen Leitfähigkeit nicht abschließend geklärt ist, gilt die Vorstellung eines hochleitfähigen Mediums, eingebettet in eine schlechtleitende Gesteinsmatrix als gesichert (VAN'YAN und HYNDMAN 1996). Für die hochleitfähige Phase werden zwei Möglichkeiten diskutiert: Salinare Fluide (Gough 1986; HYNDMAN und SHEARER 1989; KISSIN 1996) und Graphit (Frost et al. 1989; MARESCHAL et al. 1992; Zhamaletdinov 1996). Charakteristisch sind in diesem Zusammenhang oft stark richtungsabhängige Leitfähigkeiten (CULL 1985; KELLETT et al. 1992; GROOM und BAHR 1992).

Eine gute Modellvorstellung dieses Mechanismus liefern Zweiphasensysteme, in welchen die Komponenten in einfacher geometrischer Verteilung angeordnet sind. Für diese kann aus Mischungsgesetzen die effektive Leitfähigkeit des Systems angenähert, berechnet oder durch obere und untere Grenzen eingeschränkt werden. Die hohe elektrische Anisotropie ist hierbei durch eine stark richtungsabhängige Konnektivität der gutleitfähigen Phase bedingt (BAHR 1997; LABENDZ 1999).

Vielfach gehen diese Zonen erhöhter elektrischer Leitfähigkeit mit seismischen Reflexionshorizonten einher und werden gemeinsam interpretiert (HyNdman und SHeARER 1989; MERZER und KlEMPERER 1992; Simpson 1999).

Stark erhöhte, anomale Leitfähigkeiten finden sich auch im oberen Mantel (z.B. LIZARRALDE et al. 1995, HIRTH et al. 2000). Interpretiert wird hier die hohe elektrische Leitfähigkeit oft als ein mit dem Übergang zwischen Lithosphäre und Asthenosphäre einsetzendes partielles Aufschmelzen der gesteinsbildenden Materialien (JONES 1999).

Nur wenige Studien berücksichtigten dabei auch mögliche Anisotropie in der Leitfähigkeitsverteilung und waren meist auf den lithosphärischen Teil des oberen Mantels (KurTz et al. 1993; MAREschal et al. 1995) begrenzt. Nur wenige lösten anisotrope Strukturen in der oberen Asthenosphäre auf (SIMPSON 2001b; BAHR und SimPSON 2002). 
Allgemein wird davon ausgegangen, dass Olivin - mit $\sim 70 \%$ Hauptbestandteil des oberen Mantels - dessen physikalische Eigenschaften maßgeblich beeinflusst (Christensen 1984; NiCOlas und Christensen 1987; Duba und Constable 1993; Xu et al. 2000a). Unter Mantelbedingungen werden deshalb anhand von Olivin für drei mögliche Regime die Leitfähigkeiten diskutiert: für trockenen Olivin (ohne Fluiden oder Schmelze) (SHANKLAND und DubA 1990; CONSTABle 1993), für partielle Schmelzen (z.B. RoberTs und TyBURCZY 1999) oder in Anwesenheit von Wasser in Form von Wasserstoffdiffusion (KARATO 1990; HIRSCH 1990).

Allgemein wird eine Erhöhung der elektrischen Leitfähigkeit im oberen Mantel durch partielle Schmelzen begründet (LIZARRALDE et al. 1995; EvANS et al. 1999; HIRTH et al. 2000).

Der Leitfähigkeitsmechanismus durch Wasserstoffdiffusion ist durch Labormessungen (Mackwell und Kohlstedt 1990; Kohlstedt und Mackwell 1998) zwar gut verstanden, bei bisherigen Interpretationen war jedoch eine eindeutige Korrelation zwischen erhöhter Leitfähigkeit im Mantel und Wasserstoffdiffusion als deren Ursache nicht möglich.

Olivin ist bezüglich der Diffusion von Wasserstoff entlang der Hauptachsen im Kristall stark anisotrop. Entlang der Achsen unterscheiden sich die Diffusionskonstanten um bis zu zwei Größenordnungen, wobei die [100]-Achse im Kristall der Richtung hoher Diffusivität und somit Leitfähigkeit entspricht. Für isotropen Olivin mit nur geringen Wasseranteilen ist die Leitfähigkeit gegenüber trockenem Olivin nicht signifikant erhöht (CONSTABLE 1993), für ausgerichtete Kristalle hingegen ergeben sich entlang der [100]-Achse um bis zu zwei Größenordnungen höhere Leitfähigkeiten (KARATO 1990; LiZARRALDE et al. 1995). Nicht nur bezüglich der Diffusion von Wasserstoff ist der Olivinkristall anisotrop, auch die Ausbreitungsgeschwindigkeit von P- oder S-Wellen variiert entlang der Kristallachsen.

In den letzten Jahren hat die Anzahl weltweiter Beobachtungen von SKS-Scherwellensplitting stark zugenommen (WYLEGALlA et al. 1999; SAVAGE und SheEhan 2000; CURrie et al. 2001; BARruOL und Ben Ismail 2001). Als Indikator seismischer Anisotropie im oberen Mantel (SILVER 1996) wird dabei die Richtung der schnellen Scherwelle als Richtung der [100]-Achse im Olivin-Kristall interpretiert (MAINPRICE und SILVER 1993). Diese oft beobachtete großflächige Ausrichtung der Olivinkristalle sollte sich bei Anwesenheit von Wasser im oberen Mantel auch in einer signifikanten Erhöhung der elektrischen Leitfähigkeit und starken Anisotropie bemerkbar machen.

Für den oberen Mantel unter Australien konnte SIMPSON (2001) erstmals durch ein großflächiges Array bei anisotroper Leitfähigkeitsverteilung die Richtung hoher elek- 
trischer Leitfähigkeit mit der Richtung hoher seismischer Scherwellengeschwindigkeit (DEBAYle und KenNeTt 2000) korrelieren.

Die elektromagnetischen Array-Messungen im Rahmen des Eifel-Projektes ließen unter dem Rheinischen Schiefergebirge auf einen ausgedehnten, bezüglich der elektrischen Leitfähigkeit stark richtungsabhängigen oberen Mantel schließen (HöNIG 1998; LEIBECKER et al. 2002). Aufgrund der begrenzten Ausdehnung des Arrays konnte aber eine Unterscheidung zwischen anisotroper Leitfähigkeitsverteilung und zweidimensionaler Struktur nicht getroffen werden.

Im Rahmen der vorliegenden Arbeit wurde eine Erweiterung dieses Arrays durchgeführt, mit der, unter Einbeziehung früherer elektromagnetischer Messungen, nun insgesamt ein $300 \times 400$ Quadratkilometer umfassendes Array zur Verfügung steht (Kap. 2). Dieses ermöglicht Aussagen über großräumige Leitfähigkeitsverteilungen im oberen Mantel und die Unterscheidung zwischen anisotroper und zweidimensionaler Leitfähigkeit. Durch dreidimensionale Vorwärtsmodellierung wird in dieser Arbeit aus den Daten ein Leitfähigkeitsmodell bestimmt, in welchem der obere Mantel einen ausgedehnten Bereich stark anisotroper Leitfähigkeit zeigt (Kap. 3). Durch Vergleich der aus dem Modell erhaltenen Leitfähigkeiten und Anisotropie mit den Leitfähigkeitsmechanismen des oberen Mantels wird in Kap. 4 das Ergebnis der Modellierungen diskutiert. Kap. 5 widmet sich darüber hinaus dem Vergleich der elektrischen mit der seismischen Anisotropie im Messgebiet und darauf aufbauend dem Vorschlag eines Ansatzes einer gemeinsamen - durch abwechselnde Modellierung erfolgenden - Anpassung der Daten und Interpretation beider Anisotropien. 


\section{Grundlagen}

\subsection{Induktion in der Erde}

Das Magnetfeld an der Erdoberfläche trennt sich in das Hauptfeld, dessen Quellen im Erdinnern liegen und die geomagnetischen Variationen. Deren Ursprung bildet ein Stromsystem in Ionosphäre und Magnetosphäre, welches durch die von der Sonne ausgehende Teilchen- (solarer Wind) und Wellenstrahlung angeregt wird. Dieses Stromsystem ist Quelle für ein elektromagnetisches Wechselfeld, welches frequenzabhängig in die leitfähige Erde eindringt und eine Ableitung der Leitfähigkeitsverteilung im Untergrund ermöglicht.

Beschrieben wird die Ausbreitung elektromagnetischer Wellen allgemein durch die Maxwellgleichungen

$$
\begin{aligned}
\nabla \cdot \underline{D} & =q \\
\nabla \times \underline{E} & =-\partial \underline{B} / \partial t \\
\nabla \cdot \underline{B} & =0 \\
\nabla \times \underline{H} & =\underline{j}+\partial \underline{D} / \partial t,
\end{aligned}
$$

mit Elektrischem Feld $\underline{E}\left[\frac{\mathrm{V}}{\mathrm{m}}\right]$, Magnetischer Induktion $\underline{B}\left[\frac{\mathrm{Vs}}{\mathrm{m}^{2}}\right]$, Dielektrischer Verschiebung $\underline{D}\left[\frac{\mathrm{C}}{\mathrm{m}^{2}}\right]$, Magnetischer Feldstärke $\underline{H}\left[\frac{\mathrm{A}}{\mathrm{m}}\right]$, Raumladungsdichte $q\left[\frac{\mathrm{C}}{\mathrm{m}^{3}}\right]$ und Stromdichte $\underline{j}\left[\frac{\mathrm{A}}{\mathrm{m}^{2}}\right]$. Weiterhin gelten die Materialgleichungen

$$
\begin{aligned}
& \underline{B}=\mu_{0} \underline{\underline{\mu_{r}}} \underline{\underline{H}} \\
& \underline{D}=\varepsilon_{0} \underline{\underline{\varepsilon_{r}}} \underline{\underline{E}}
\end{aligned}
$$

und das Ohmsche Gesetz

$$
\underline{j}=\underline{\underline{\sigma}} \underline{E}
$$

mit relativer und absoluter Permeabilität $\underline{\underline{\mu_{r}}}, \mu_{0}\left[\frac{\mathrm{Vs}}{\mathrm{Am}}\right]$ und relativer und absoluter Dielektrizitätskonstante $\underline{\underline{\varepsilon_{r}}}, \varepsilon_{0}\left[\frac{\mathrm{As}}{\mathrm{Vm}}\right]$. Die elektrische Leitfähigkeit ${ }^{1} \underline{\underline{\sigma}}\left[\frac{\mathrm{S}}{\mathrm{m}}\right]$ verknüpft

\footnotetext{
${ }^{1}$ Gebräuchlicher ist in der elektromagnetischen Tiefenforschung der spezifische elektrische Widerstand mit $\rho=\sigma^{-1}[\mathrm{Vm} / \mathrm{A}=\Omega \mathrm{m}]$.
} 
im quellfreien Medium im Ohmschen Gesetz Stromdichte und elektrisches Feld. Im Folgenden wird zunächst davon ausgegangen, dass die elektrische Leitfähigkeit ein Skalar $\underline{\sigma} \rightarrow \sigma$ ist. Eine Erweiterung auf den allgemeinen Fall des Leitfähigkeitstensors erfolgt in Kap. 1.4.

Die Maxwellgleichungen sind partielle Differentialgleichungen, die allgemein das raumzeitliche Verhalten elektromagnetischer Felder in beliebigen Medien beschreiben. Für den im Folgenden verwendeten Periodenbereich und die in der Erde vorherrschenden Leitfähigkeitsverhältnisse können sie durch einige Näherungen vereinfacht werden. Das elektromagnetische Feld dringt als quasistationäre ebene Welle in die Erde ein, d.h. Verschiebungsströme $(\partial \underline{D} / \partial t=0)$ können vernachlässigt werden, die Anzahl der (stationären) freien Ladungsträger wird Null gesetzt. Für die Erde können relative Dielektrizitätszahl $\varepsilon_{r}$ und magnetische Permeabilität $\mu_{r}$ als richtungsunabhängig und konstant angenommen werden: $\mu_{r}, \varepsilon_{r} \approx 1$. Durch Fouriertransformation der Feldgrößen geht die Zeitabhängigkeit in eine Frequenzabhängigkeit $(\omega=2 \pi / T)$ über und die Feldgrößen ergeben sich als Überlagerung komplexer harmonischer Schwingungen $e^{-i \omega t}$. Damit entkoppeln die Maxwellgleichungen und man erhält die Helmholtzgleichungen

$$
\nabla^{2} \underline{F}=i \omega \mu_{0} \sigma \underline{F}
$$

mit $\underline{F}=\underline{E}, \underline{B}$. Sie haben die Form einer Diffusionsgleichung. Für den homogenen Halbraum erhält man daraus die Skin- oder Eindringtiefe $p$. Sie gibt die Tiefe an, bei der das eindringende Feld auf den Faktor 1/e abgeklungen ist:

$$
p[\mathrm{~m}]=\sqrt{\frac{2}{\mu_{0} \sigma \omega}} \Longrightarrow p[\mathrm{~km}] \approx \frac{1}{2} \sqrt{\rho T} .
$$

\subsection{Magnetotellurik}

Die Magnetotellurik (MT) ist ein elektromagnetisches Prospektionsverfahren zur Bestimmung der Tiefenverteilung der elektrischen Leitfähigkeit. In der MT werden die natürlichen elektrischen und magnetischen Feldvariationen an der Erdoberfläche gemessen. Die zwischen $\mathrm{E}$ und B bestimmte frequenzabhängige Übertragungsfunktion wird magnetotellurische Impedanz $z^{2}$ genannt. Ihre komplexen Einträge enthalten Informationen über die Leitfähigkeitsverteilung im Untergrund.

Allgemein besteht zwischen horizontalem elektrischem und magnetischem Feld ${ }^{3}$ an der

\footnotetext{
${ }^{2}$ Auch Impedanztensor oder verkürzt Impedanz genannt.

${ }^{3}$ Gemessen im in der Geophysik üblichen Koordinatensystem mit x: magnetisch Nord, y: magnetisch Ost und $\mathrm{z}$ : positiv nach unten.
} 
Erdoberfläche im Frequenzbereich die Beziehung

$$
\left(\begin{array}{c}
E_{x} \\
E_{y}
\end{array}\right)=\underbrace{\left(\begin{array}{ll}
Z_{x x} & Z_{x y} \\
Z_{y x} & Z_{y y}
\end{array}\right)}_{=\underline{\underline{Z}}}\left(\begin{array}{l}
B_{x} \\
B_{y}
\end{array}\right),
$$

mit der komplexen Übertragungsfunktion $\underline{\underline{Z}}$, deren Form sich aus der Verteilung der Leitfähigkeiten bestimmt. Es kann zwischen drei Fällen unterschieden werden:

3D-Fall Für eine dreidimensionale Verteilung der elektrischen Leitfähigkeit im Untergrund $\sigma=\sigma(x, y, z)$ ist der Impedanztensor vollbesetzt.

2D-Fall Es sei $\sigma=\sigma(x, z)$. In y-Richtung (Streichrichtung) ist die Leitfähigkeit konstant. Im Koordinatensystem des Streichens hat der Impedanztensor Nebendiagonalform, $Z_{x x}=Z_{y y}=0$. Die Nebendiagonalelemente sind vom Betrag verschieden, $\left|Z_{x y}\right| \neq\left|Z_{y x}\right|$.

1D-Fall Eindimensionale Leitfähigkeitenverteilungen sind der homogene ( $\sigma=$ konst.) oder der homogen geschichtete Halbraum $(\sigma=\sigma(z))$. Für den geschichteten Halbraum verschwinden (vgl. '2D-Fall') die Hauptdiagonalelemente, zusätzlich gilt: $Z_{x y}=-Z_{y x}=Z_{n}$. Beim geschichteten Halbraum ist $Z_{n}$ frequenzabhängig, über einem homogenen Halbraum konstant.

Aus den Einträgen im Impedanztensor lassen sich die Größen scheinbarer spezifischer Widerstand, $\rho_{a}$ und Phase $\phi$ ableiten:

$$
\begin{aligned}
\rho_{a i j} & =\frac{\mu_{0}}{\omega}\left|Z_{i j}\right|^{2} \\
\phi_{i j} & =\arg Z_{i j}=\arctan \left\{\operatorname{Im}\left(Z_{i j}\right) / \operatorname{Re}\left(Z_{i j}\right)\right\} .
\end{aligned}
$$

Für den geschichteten Halbraum wird mit (1.4) jeder Frequenz des Impedanztensors formal ein äquivalenter homogener Halbraum mit spezifischem Widerstand $\rho_{a}$ zugeordnet

$$
\rho_{a}=\mu_{0}\left|Z_{n}\right|^{2} / \omega \text {. }
$$

Für den homogenen Halbraum gibt der scheinbare spezifische Widerstand den wahren Widerstand des Halbraumes wieder (CAGNIARD 1953). Die Phase der Einträge im Impedanztensor $\phi$ (1.5) sind im geschichteten Halbraum die Phase zwischen den Komponenten des elektrischen und magnetischen Feldes. Im Fall des homogenen Halbraums ist $\phi$ konstant, $\phi=45^{\circ}$. Im geschichteten Halbraum zeigt entsprechend der frequenzabhängigen Eindringtiefe $\phi>45^{\circ}$ einen guten Leiter unter einem schlechten und $\phi<45^{\circ}$ den umgekehrten Fall an (SCHMUCKER 1987). 
Ausgehend von einem Zweischichtmodell eines Halbraums mit Deckschicht entwickelte SCHMUCKER (1987) die $\rho^{*}$-z $z^{*}$-Transformation. Hierbei wird der spezifische Widerstand $\rho^{*}$ gegen die Tiefe $z^{*}$ aufgetragen, die als Schwerpunkttiefe der induzierten Ströme interpretiert werden kann (WEIDELT 1972):

$$
\rho^{*}=\left\{\begin{array}{ll}
2 \cdot \rho_{a} \cdot \cos ^{2} \phi & \text { für } 0^{\circ} \leq \phi \leq 45^{\circ} \\
\frac{\rho_{a}}{2 \cdot \sin ^{2} \phi} & \text { für } 45^{\circ} \leq \phi \leq 90^{\circ}
\end{array} \quad \text { und } \quad z^{*}=\operatorname{Re}\left(\frac{Z}{i \omega}\right) .\right.
$$

Bei dieser Widerstands-Tiefenauftragung geht die Phase mit in die Darstellung ein. Die Auftragung von $\rho^{*}\left(z^{*}\right)$ liefert eine gute Näherung der Eindringtiefe des Feldes bei entsprechender Frequenz und der wahren Widerstandsverhältnisse in entsprechender Tiefe.

\section{Eine einfache Modellvorstellung}

Für die Magnetotellurik sind zweidimensionale Leitfähigkeitsstrukturen von großer Bedeutung, da sie für die Interpretation von Messdaten eine wichtige Modellvorstellung sind.

Gegeben sei eine zweidimensionale Verteilung der elektrischen Leitfähigkeit $\sigma=\sigma(x, z)$. Da die Ableitungen der Felder in y-Richtung verschwinden $(\partial F / \partial y=0)$, entkoppeln die Maxwellgleichungen in zwei unabhängige Gleichungssysteme. Die sog. xy-Polarisation entspricht den in Streichrichtung, die $y x$-Polarisation den senkrecht dazu fließenden Strömen. Im Koordinatensystem des Streichens hat der Impedanztensor (1.3) Nebendiagonalform:

$$
\underline{\underline{Z}}=\left(\begin{array}{cc}
0 & Z_{x y} \\
Z_{y x} & 0
\end{array}\right) .
$$

Aus (1.4) und (1.5) erhält man für jede der beiden Polarisationen eine $\rho_{a^{-}}$und Phasenkurve.

Die einfache Form (1.7) gilt allerdings nur im Koordinatensystem des Streichens. Weicht das Koordinatensystem, in dem die Daten gemessen wurden davon ab, ist der Impedanztensor vollbesetzt. Durch Rotation kann die Impedanz in das Koordinatensystem des Streichens ${ }^{4}$ überführt werden. Den gedrehten Tensor $\underline{\underline{Z^{\prime}}}$ erhält man aus dem gemessenen $\underline{\underline{Z}}$ durch die Transformation:

$$
\underline{\underline{Z}}^{\prime}=\underline{\underline{D}}^{\mathrm{T}} \cdot \underline{\underline{Z}} \cdot \underline{\underline{D}}
$$

mit der Drehmatrix

$$
\underline{\underline{D}}=\left(\begin{array}{cc}
\cos \alpha_{s} & -\sin \alpha_{s} \\
\sin \alpha_{s} & \cos \alpha_{s}
\end{array}\right)
$$

\footnotetext{
${ }^{4}$ Im Folgenden beschreiben ungestrichene Koordinaten im geophysikalischen Koordinatensystem gemessene Daten, gestrichene beziehen sich auf das gedrehte Koordinatensystem.
} 
Der Drehwinkel $\alpha_{s}$ berechnet sich aus der Minimierung ${ }^{5}:\left|Z_{x x}^{\prime}\right|^{2}+\left|Z_{y y}^{\prime}\right|^{2} \rightarrow$ Min. (SWIFT 1967). Aus dieser Bedingung folgt für den Swiftwinkel:

$$
\alpha_{s}=\frac{1}{4} \arctan \left(\frac{2 \cdot \operatorname{Re}\left\{S_{2} D_{1}^{*}\right\}}{\left|D_{1}\right|^{2}+\left|S_{2}\right|^{2}}\right)
$$

mit den modifizierten Impedanzen

$$
\begin{array}{ll}
S_{1}=Z_{x x}+Z_{y y} & S_{2}=Z_{x y}+Z_{y x} \\
D_{1}=Z_{x x}-Z_{y y} & D_{2}=Z_{x y}-Z_{y x} .
\end{array}
$$

Als Schiefe des Impedanztensors $\kappa$ gab SWIFT (1967) ein rotationsinvariantes Maß für Abweichungen vom zweidimensionalen Fall an:

$$
\kappa=\frac{\left|Z_{x x}+Z_{y y}\right|}{\left|Z_{x y}-Z_{y x}\right|} .
$$

Im Fall einer 1D- oder 2D-Leitfähigkeitsverteilung ist sie Null. Ist $\kappa$ groß, ist die Leitfähigkeitsverteilung unter der Station dreidimensional. Das dies jedoch kein eindeutiges Kriterium zur Klärung der Dimensionalität ist, haben TING und HOHMANN (1981) durch Modellstudien gezeigt: Auch dreidimensionale Modelle können über Symmetrieachsen kleine Schiefen aufweisen.

\subsubsection{Verzerrung}

Oberflächennahe, lokale Anomalien, können das elektromagnetische Feld beeinflussen und die Messung der regionalen ${ }^{6}$ Leitfähigkeitsstruktur verfälschen. Dieser als Verzerrung ${ }^{7}$ bezeichnete Effekt hat seine Ursache im an den Störkörpern gestreuten elektromagnetischen Feld (WEAVER 1994). Ist die Skintiefe groß im Vergleich zur Lage des anomalen Störkörpers, ist der induktive Anteil vernachlässigbar (GROOM und BAHR 1992; Chave und SMith 1994). Für das verzerrte elektrische Feld $\underline{E}$ folgt

$$
\underline{E}=\underline{\underline{C}} \cdot \underline{E}^{r},
$$

mit dem regionalen elektrischen Feld $\underline{E}^{r}$ und der reellen, frequenzunabhängigen Verzerrungsmatrix $\underline{\underline{C}}$. Analog erhält man für das Magnetfeld $\underline{B}$

$$
\underline{B}=\underline{B}^{r}+\underline{D} \cdot \underline{E}^{r},
$$

\footnotetext{
${ }^{5} \operatorname{Im}$ idealisierten 2D-Modell ist die Bedingung $\left|Z_{x x}^{\prime}\right|^{2}+\left|Z_{y y}^{\prime}\right|^{2}=0$. Diese wird bei realen Daten durch die Minimierung ersetzt, da der reine 2D-Fall in der Natur keine Realisierung findet und die Messdaten fehlerbehaftet sind.

${ }^{6}$ Unter regional ist hier die großräumige Leitfähigkeitsstruktur zu verstehen, wie sie sich ohne Einfluss oberflächennaher Störkörper zeigen würde.

${ }^{7}$ Da es sich hierbei um einen Gleichstrom-Effekt handelt (vgl. z.B. VozofF 1987), wird er auch als galvanische Verzerrung bezeichnet.
} 
mit der reellen, frequenzunabhängigen magnetischen Verzerrungsmatrix $\underline{\underline{D}}$ (Chave und SMith 1994; SMith 1997). Das anomale Magnetfeld $\underline{\underline{D}} \cdot \underline{E^{r}}$ entsteht dabei durch räumliche Variation des regionalen elektrischen Feldes und ist somit mit diesem in Phase (RITTER 1996). Der Einfluss der magnetischen Verzerrung ist im Vergleich zur elektrischen gering und vielfach zu vernachlässigen (GROOM und BAILEY 1991). In Anwesenheit sehr starker kanalisierter Ströme durch starke laterale Inhomogenitäten, sog. 'current channeling' muss er mit berücksichtigt werden (LEZAETA 2001). In den in dieser Arbeit verwendeten Daten ist dies nicht zu beobachten und die magnetische Verzerrung kann daher vernachlässigt werden.

Die Überlagerung einer regionalen zweidimensionalen Leitfähigkeitsverteilung und eines lokalen, oberflächennahen Störkörpers wird im Koordinatensystem des Streichens durch den Impedanztensor

$$
\underline{\underline{Z}}=\underline{\underline{A}} \cdot \underline{\underline{Z^{\prime}}}=\left(\begin{array}{ll}
a_{11} & a_{12} \\
a_{21} & a_{22}
\end{array}\right)\left(\begin{array}{cc}
0 & Z_{x y}^{\prime} \\
Z_{y x}^{\prime} & 0
\end{array}\right)=\left(\begin{array}{ll}
a_{12} Z_{y x}^{\prime} & a_{11} Z_{x y}^{\prime} \\
a_{22} Z_{y x}^{\prime} & a_{21} Z_{x y}^{\prime}
\end{array}\right)
$$

beschrieben, hierbei ist $\underline{\underline{Z}}^{\prime}$ der unverzerrte regionale Tensor im Koordinatensystem des Streichens und $\underline{\underline{A}}$ die reelle, den Impedanztensor beeinflussende Verzerrungsmatrix (BAHR 1988).

Durch Multiplikation mit der reellen Verzerrungsmatrix $\underline{\underline{A}}$ wird aus der Nebendiagonalform im Koordinatensystem der 2D-Struktur (1.7) ein vollbesetzter Tensor. Die Verzerrungmatrix ändert bei den Nebendiagonalelementen die Amplitude der Einträge, die Phase bleibt unbeeinflusst

$$
\begin{aligned}
& \rho_{a x y}^{\prime}=\frac{\mu_{0}}{\omega}\left|Z_{x y}^{\prime}\right|^{2}=a_{11}^{2} \cdot \rho_{a x y} \\
& \phi_{x y}^{\prime}=\arg Z_{x y}^{\prime}=\phi_{x y} .
\end{aligned}
$$

Die hieraus resultierende Änderung der $\rho_{a}$-Werte wird als static shift ${ }^{8}$ bezeichnet.

Die Spalten des Impedanztensors geben dabei den mit $B_{x}$ bzw. $B_{y}$ korrelierten Anteil des elektrischen Feldes an

$$
\underline{t}_{x}=\left(\begin{array}{l}
a_{12} Z_{y x}^{\prime} \\
a_{22} Z_{y x}^{\prime}
\end{array}\right) \quad \underline{t}_{y}=\left(\begin{array}{l}
a_{11} Z_{x y}^{\prime} \\
a_{21} Z_{x y}^{\prime}
\end{array}\right)
$$

$\underline{t}_{x}, \underline{t}_{y}:$ tellurische Vektoren.

\footnotetext{
${ }^{8}$ Der scheinbare spezifische Widerstand wird logarithmisch aufgetragen: $\log \left(\rho_{a_{x y}}^{\prime}\right)=\log \left(a_{22}^{2}\right)+\log \left(\rho_{a_{x y}}\right)$. Dabei bewirkt der Verzerrungsfaktor eine über den Periodenbereich konstante Verschiebung der $\rho_{a^{-}}$ Kurve.
} 


\subsubsection{Strukturbestimmung}

Bedingt durch die Verzerrung des elektrischen Feldes hat der Impedanztensor im Koordinatensystem des Streichens nicht die Nebendiagonalform. Das auf SWIFT zurückgehende Drehkriterium (Kap. 3) führt damit nicht zum gesuchten Ergebnis.

Da die Verzerrungsmatrix $\underline{\underline{A}}$ nur reelle Einträge enthält, haben im Koordinatensystem des Streichens die Spalten des Impedanztensors (1.9) gleiche Phase. Dies liefert eine neue analytische Drehbedingung. Für den phasensensitiven Drehwinkel folgt

$$
\alpha_{B}=\frac{1}{2} \arctan \left(\frac{\left[S_{1}, S_{2}\right]-\left[D_{1}, D_{2}\right]}{\left[S_{1}, D_{1}\right]-\left[S_{2}, D_{2}\right]}\right)
$$

mit den in (1.8) definierten modifizierten Impedanzen ${ }^{9}$. Wie für den Fall der reinen 2DStruktur wird auch für den Fall der Superposition mit einer lokalen 3D-Anomalie eine rotationsinvariante Maßzahl

$$
\eta=\frac{\sqrt{\left[D_{1}, S_{2}\right]-\left[S_{1}, D_{2}\right]}}{\left|D_{2}\right|}
$$

definiert (BAHR 1988). Anders als bei SWIFT werden hier jedoch die Phasen der Impedanz ausgewertet. Die Schiefe $\eta$ gibt die Abweichung vom vorausgesetzten 2D-Modell an. Eine direkte Zuordung zwischen Schiefe und Modellkomplexität ist jedoch nicht möglich (BAHR 1991). Ist $\eta>0.3$ muss davon ausgegangen werden, dass die regionale Leitfähigkeitsstruktur nicht mehr 2D ist. Der Umkehrschluss ist nicht zulässig, da auch 3D-Modelle kleine phasensensitive Schiefen aufweisen können!

In realen Daten sind die obigen Voraussetzungen nur näherungsweise erfüllt. Durch sich vertikal oder horizontal ändernde Leitfähigkeiten ist die regionale Struktur nicht mehr zweidimensional. Lediglich abschnittsweise kann diese mit der Tiefe angenähert als zweidimensional angesehen werden. Ebenso kann nicht mehr vereinfachend davon ausgegangen werden, dass nur oberflächennahe, kleinräumige Leitfähigkeitsanomalien das elektrische Feld statisch verzerren. Strukturen in der Kruste, die von kurzen Perioden noch induktiv wahrgenommen werden, wirken bei langen Perioden und damit größeren Eindringtiefen als statische Verzerrer (SIMPSON und WARNER 1998). Der Verzerrungstensor ist somit nicht mehr frequenzunabhängig (LEDO et al. 1998).

Stimmen die phasensensitiven Rotationswinkel benachbarter Stationen trotz großer phasensensitiver Schiefen überein, kann die regionale Leitfähigkeitsstruktur als annähernd (oder gemittelt) 2D angesehen werden (BAHR 1991). Ist der phasensensitive Rotationswinkel darüber hinaus frequenzabhängig, liegt möglicherweise eine Überlagerung von Strukturen mit richtungsabhängiger, zweidimensionaler oder anisotroper Leitfähigkeit vor (vgl. Abb. 1.1). Die Übertragungsfunktion kann dann für jede Frequenz

$$
{ }^{9}[A, B]=\operatorname{Re}\{A\} \operatorname{Im}\{B\}-\operatorname{Im}\{A\} \operatorname{Re}\{B\}
$$




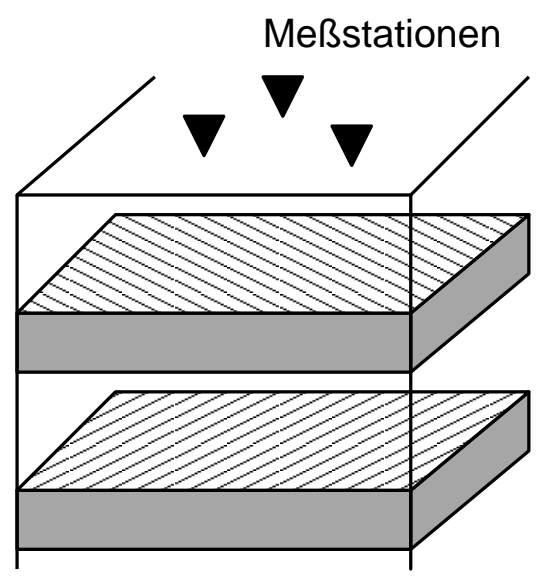

Abbildung 1.1: Einfaches Modell der Überlagerung abschnittsweise richtungsabhängiger Leitfähigkeiten.

näherungsweise als Überlagerung einer reellen Verzerrungsmatrix und einer im Mittel zweidimensionalen (richtungsabhängigen) Leitfähigkeitsstruktur angenommen werden (BAHR et al. 2000).

Einen ähnlichen Ansatz bei der Beschreibung von Verzerrung wählten GroOM und BAILEY (1989). Basierend auf der gleichen Modellvorstellung wie bei BAHR (1988) erhalten sie durch eine Parametrisierung und Zerlegung des Verzerrungstensors das Koordinatensystem des 2D-Streichens. Die Berechnung der Parameter erfolgt dabei numerisch. Durch Erweiterung dieses Ansatzes kann hierbei auch der Effekt magnetischer Verzerrung (Zhang et al. 1993; Chave und Smith 1994; SMith 1997) mit berücksichtigt werden.

Allein mit langperiodischen magnetischen Feldern ist es nach dem Z:H-Verfahren (ECKHARDT 1963; SOYER 1998) möglich, eine skalare, magnetische Impedanz zu berechnen. Da die magnetischen Felder weitgehend frei von statischen Verzerrungen sind, ist hiermit eine Möglichkeit gegeben, aus dem Vergleich dieser magnetischen und der magnetotellurischen Impedanz, den Anteil der Verzerrung zu berechnen (BAHR 1985). Diese Entzerrung der Impedanz für lange Perioden liefert die frequenzunabhängige Verzerrungsmatrix (1.9). Ist die gemessene magnetotellurische Impedanz durch frequenzabhängige Verzerrung beeinflusst, ist die Entzerrung nicht, oder nur als Näherung möglich.

\subsection{Erdmagnetische Tiefensondierung}

Im Unterschied zur Magnetotellurik werden bei der Erdmagnetischen Tiefensondierung die Übertragungsfunktionen nur aus magnetischen Komponenten abgeleitet. Grundlage 
für die Methode ist die Vorstellung, dass sowohl die Leitfähigkeitsverteilung, als auch elektrisches und magnetisches Feld in einen normalen und einen anomalen Anteil zerlegt werden können. Die Leitfähigkeitsverteilung $\sigma$ setzt sich aus einer geschichteten Normalstruktur $\sigma^{n}$ und einem anomalen Anteil $\sigma^{a}$ zusammen: $\sigma=\sigma^{n}(z)+\sigma^{a}(x, y, z)$. Für die Felder folgt: $\underline{F}=\underline{F}^{n}+\underline{F}^{a}(F=E, B)$.

Die gemessenen magnetischen Feldkomponenten werden auf eine Referenzstation über möglichst eindimensionaler Leitfähigkeitsverteilung bezogen. Mit diesen Normalfeldern folgt:

$$
\left(\begin{array}{c}
B_{x}^{a} \\
B_{y}^{a} \\
B_{z}^{a}
\end{array}\right)=\underline{\underline{W}}\left(\begin{array}{c}
B_{x}^{n} \\
B_{y}^{n}
\end{array}\right)
$$

mit der normalen Übertragungsfunktion $\underline{\underline{W}}$ dem sog. Perturbationstensor (SCHMUCKER 1970).

Über einem geschichteten Halbraum gibt es kein vertikales Magnetfeld: $B_{z}^{n}=0$. Demnach ist eine Unterteilung der vertikalen Komponente in normalen und anomalen Anteil wenig sinnvoll, $B_{z}=B_{z}^{a}$. Somit können die Horizontalkomponenten des Magnetfeldes $B_{x, y}$ auch direkt auf die vertikale Komponente bezogen werden. Man erhält lokale, frequenzabhängige Übertragungsfunktionen $z_{H}^{l}$ und $z_{D}^{l}$ aus

$$
B_{z}=z_{H}^{l} B_{x}+z_{D}^{l} B_{y} .
$$

Diese Übertragungsfunktionen für das vertikale Magnetfeld können durch Induktionspfeile dargestellt werden. Die aus den Realteilen von $z_{H}^{l}$ und $z_{D}^{l}$ gebildeten Pfeile werden Realpfeile, die aus den Imaginärteilen Imaginärpfeile genannt:

$$
\text { Realpfeil }=\left(\begin{array}{l}
\operatorname{Re}\left\{z_{H}^{l}\right\} \\
\operatorname{Re}\left\{z_{D}^{l}\right\}
\end{array}\right), \quad \text { Imaginärpfeil }=\left(\begin{array}{c}
\operatorname{Im}\left\{z_{H}^{l}\right\} \\
\operatorname{Im}\left\{z_{D}^{l}\right\}
\end{array}\right) .
$$

Werden sie über einem Leitfähigkeitskontrast flächenhaft aufgetragen, so zeigen sie vom guten Leiter weg (WIESE 1962).

\subsection{Anisotropie der Leitfähigkeit}

In Kap. 1.2 wird davon ausgegangen, dass die elektrische Leitfähigkeit ein Skalar ist. Richtungsabhängigkeiten der Leitfähigkeit treten dabei nur durch sich lateral ändernde Leitfähigkeiten, im allgemeinen an 2D oder 3D Leitfähigkeitskontrasten, auf. Bei der Modellvorstellung einer 2D-Struktur in Form eines Leitfähigkeitskontrastes hat der Impedanztensor nur in der Nähe des Kontrastes die Form (1.7). Mit zunehmender Entfernung vom Kontrast nähert sich der Tensor der eindimensionalen Form an.

Messen an der Oberfläche mehrere Stationen die gleiche Richtungsabhängigkeit der Leitfähigkeit, ändert sie sich also lateral nicht, so spricht man von Anisotropie der elektri- 

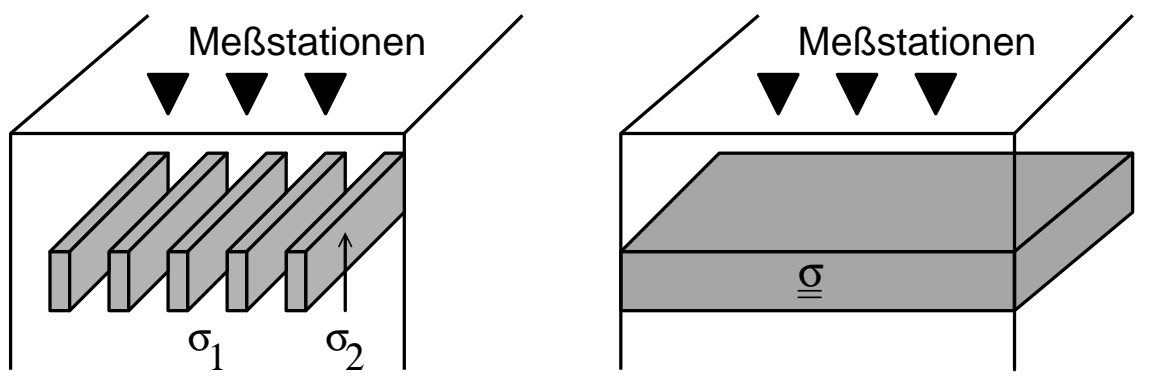

Abbildung 1.2: Modellvorstellung der makroskopischen (links) und mikroskopischen (rechts) Anisotropie. Links: Lamellen der Leitfähigkeit $\sigma_{2}$ sind eingebettet in den Hintergrund der Leitfähigkeit $\sigma_{1}$. Rechts: Die anisotrope Schicht hat eine Leitfähigkeit $\underline{\underline{\sigma}}$ der Form (1.14) (nach LEIBECKER 2000).

schen Leitfähigkeit. Zur Erklärung dieser Anisotropie kann auf zwei Modellvorstellungen zurückgegriffen werden, die makroskopische und die mikroskopische oder intrinsische Anisotropie. In Abb. 1.2 sind beide vereinfacht dargestellt. Bei der makroskopischen Anisotropie wird die Richtungsabhängigkeit durch zweidimensionale Strukturen erzeugt, die in den Hintergrund eingebettet sind. Diese bedingen eine Vorzugsrichtung des Stromflusses entlang der gutleitenden Lamellen. Mit Methoden der Magnetotellurik allein kann zwischen beiden Arten der Anisotropie nicht unterschieden werden (EISEL und HAAK 1999).

Allgemein ist die Leitfähigkeit kein Skalar sondern wird beschrieben durch einen $(3 \times 3)$-Tensor. Da der Leitfähigkeitstensor $\underline{\underline{\sigma}}$ positiv definit und symmetrisch ist, kann er durch Hauptachsentransformation auf Diagonalgestalt gebracht werden (DEKKER und Hastie 1980; MAURer 1993):

$$
\underline{\underline{\sigma}}^{\prime}=\left(\begin{array}{ccc}
\sigma_{x}^{\prime} & 0 & 0 \\
0 & \sigma_{y}^{\prime} & 0 \\
0 & 0 & \sigma_{z}^{\prime}
\end{array}\right) .
$$

Die Kenntnis der Leitfähigkeiten entlang der Hauptachsen im Kristall ist für die Beschreibung der Leitfähigkeiten von Gesteinen von entscheidender Bedeutung. Auf Leitfähigkeiten, ihre Mechanismen und daraus resultierende Anisotropien wird in Kap. 4 detaillierter eingegangen. 


\section{Elektromagnetische Arraymessungen}

\subsection{Feldmessungen}

Das Gebiet zwischen Rheinischem Schiefergebirge, Fränkischem Schichtstufenland und bis hinauf zur Norddeutschen Tiefebene war in den letzten zwei Jahrzehnten oft Gegenstand elektromagnetischer Messungen. In zahlreichen Diplom- sowie Doktorarbeiten sind einzelne Stationen oder Profile vermessen und interpretiert worden (Fluche 1983; BAHR 1985; PeTER 1994; BANTIN 1996; CyPliK 1996; SCHNEIDER 1998; BAHR und DUBA 2000; BAHR et al. 2000). Insgesamt liegen aus diesen Arbeiten magnetotellurische Daten von 30 Stationen vor.

Im Rahmen des Eifel-Projektes ist im Rheinischen Schiefergebirge im Sommer 1997 und Winter 1998 ein Array von 21 MT-Stationen vermessen und ausgewertet worden (LEIBECKER 2000). In Abb. 2.1 sind diese 51 Stationen durch schwarze Dreiecke dargestellt. Um diese Stationen als ein Array gemeinsam interpretieren zu können, wurde im Rahmen dieser Arbeit in zwei Feldexperimenten im Sommer 1999 und 2000 das Messgebiet durch weitere 13 Stationen (rote Dreiecke in Abb. 2.1) erweitert. Bestehende Lücken zwischen den alten Stationen wurden dabei geschlossen und das Array nach Norden ausgedehnt.

\section{Messapparatur}

Zur Registrierung der elektrischen und magnetischen Felder wurde das am Institut für Geophysik in Göttingen entwickelte RAP-System verwendet (Steveling und LeVen 1994; Steveling 1996). Es ermöglicht die Aufzeichnung elektrischer und magnetischer Variationen für Perioden $>10 \mathrm{~s}$. Die Bestimmung des elektrischen Feldes erfolgt über die Messung der Potentialdifferenz zwischen zwei unpolarisierbaren, langzeitstabilen Ag-AgCl-Elektroden (FILlOUX 1987), die im Mindestabstand von $50 \mathrm{~m}$ ca. $20 \mathrm{~cm}$ tief in der Erde vergraben werden. Die Messung der Magnetfelder erfolgt mit Fluxgate-Magnetometern. 


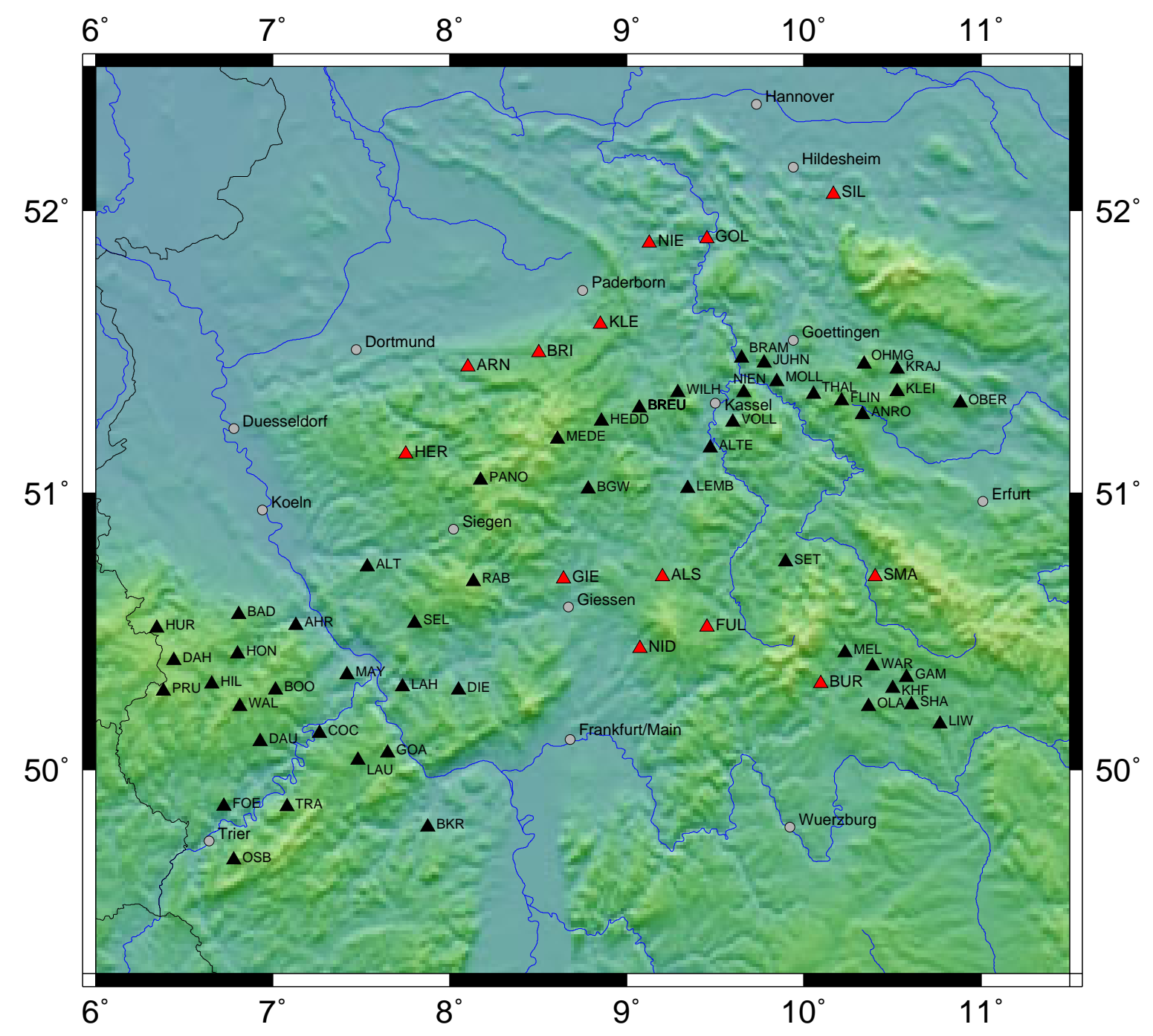

Abbildung 2.1: Karte des Messgebietes mit 64 MT-Stationen. Die schwarzen Dreiecke zeigen die Koordinaten der in früheren Arbeiten vermessenen Stationen. In rot dargestellt, die im Rahmen dieser Arbeit vermessenen Stationen.

\section{Messkampagnen}

Während der Messkampagnen kamen Geräte des Göttinger Instituts für Geophysik sowie von der Freien Universität Berlin zur Verfügung gestellte Stationen zum Einsatz. 11 der 13 Stationen registrierten zwischen dem 29.07. und 15.09.99. Eine Erweiterungsmessung im Zeitraum vom 17.05. bis 13.06.00 umfasste zwei zusätzliche Stationen, sowie die Wiederholungsmessung einer Station. Eine Übersicht über die Stationen, Registrierzeiträume und deren Koordinaten ist dem Anhang A.1 zu entnehmen. 
Insgesamt steht somit ein Datensatz aus 64 MT-Stationen zur Verfügung, der eine Fläche von $300 \times 400$ Quadratkilometern überdeckt.

\subsection{Datenanalyse}

Durch die Datenanalyse wird die Übertragungsfunktion im Frequenzbereich (1.3) aus den im Zeitbereich gemessenen elektrischen und magnetischen Feldgrößen ermittelt.

Aus den aufgezeichneten Zeitreihen werden zunächst die Bereiche, die von Störungen unbeeinflusst sind ausgewählt und durch Fouriertransformation in den Frequenzbereich überführt. Eine Auswertung erfolgt an festgelegten Auswertefrequenzen $f_{e}$, die so gewählt sind, dass bei logarithmischer Auftragung die Punkte annähernd äquidistant sind. In Tabelle 2.1 sind die bei der Auswertung verwendeten Frequenzen aufgelistet ${ }^{1}$.

\begin{tabular}{|r|c||r|c|}
\hline$T_{e}(\mathrm{~s})$ & $f_{e}(\mathrm{~Hz})$ & $T_{e}(\mathrm{~s})$ & $f_{e}(\mathrm{~Hz})$ \\
\hline \hline 8196 & $1.22 \cdot 10^{-4}$ & 256 & $3.91 \cdot 10^{-3}$ \\
5464 & $1.83 \cdot 10^{-4}$ & 171 & $5.84 \cdot 10^{-3}$ \\
4096 & $2.44 \cdot 10^{-4}$ & 128 & $7.81 \cdot 10^{-3}$ \\
2731 & $3.66 \cdot 10^{-4}$ & 85 & $1.18 \cdot 10^{-2}$ \\
2048 & $4.88 \cdot 10^{-4}$ & 64 & $1.56 \cdot 10^{-2}$ \\
1365 & $7.33 \cdot 10^{-4}$ & 43 & $2.33 \cdot 10^{-2}$ \\
1024 & $9.77 \cdot 10^{-4}$ & 32 & $3.13 \cdot 10^{-2}$ \\
683 & $1.46 \cdot 10^{-3}$ & 21 & $4.76 \cdot 10^{-2}$ \\
512 & $1.95 \cdot 10^{-3}$ & 16 & $6.25 \cdot 10^{-2}$ \\
341 & $2.93 \cdot 10^{-3}$ & 11 & $9.09 \cdot 10^{-2}$ \\
\hline
\end{tabular}

Tabelle 2.1: Für die Analyse festgelegte Auswertefrequenzen.

Die Einträge des Impedanztensors werden durch bivariate Analyse an diesen Auswertefrequenzen $f_{e}$ bestimmt:

$$
\begin{aligned}
& E_{x}=Z_{x x} B_{x}+Z_{x y} B_{y}+\Delta E_{x} \\
& E_{y}=Z_{y x} B_{x}+Z_{y y} B_{y}+\Delta E_{y} .
\end{aligned}
$$

Annahme hierbei ist, dass nur das elektrische Feld fehlerbehaftet ist. Die analytische Form zur Berechnung der $Z_{i j}$ folgt aus der Minimierungsbeziehung des Fehlers: $\Delta E_{i} \rightarrow$ Min. Eine detaillierte Darstellung der Analyse und die Vorstellung der verwendeten Program-

\footnotetext{
${ }^{1}$ Die Auswertefrequenzen sind nicht bei allen in dieser Arbeit verwendeten Übertragungsfunktionen identisch. Lediglich die im Rahmen dieser Arbeit und des Eifel-Projektes (LEIBECKER 2000) gemessenen Stationen haben Übertragungsfunktionen für die in Tab. 2.1 aufgelisteten Frequenzen.
} 
me findet sich bei BAHR and BRUTON (1994) sowie JANTOS (1998). Im Anhang A.2 ist eine Übersicht der zur Selektion und Analyse verwendeten Programme enthalten.

\subsection{Ergebnisse der Datenanalyse}

In diesem Kapitel werden aus den berechneten Übertragungsfunktionen durch geeignete Betrachtung der darin enthaltenen (in Kapitel 1 diskutierten) geophysikalischen Größen, Informationen über die Tiefenverteilung der elektrischen Leitfähigkeit im Messgebiet gewonnen. Zur übersichtlichen Darstellung erfolgt die Betrachtung der Charakteristika in den Daten anhand einzelner Stationen oder Stationsgruppen. Für den westlichen Teil des Messgebietes sind aufgrund guter Datenqualität die in Abb. 2.2 dargestellten 8 Stationen ausgewählt. Die Station DIE steht hierbei stellvertretend für alle westlich davon gelegenen Stationen im Rheinischen Schiefergebirge (LEIBECKER 2000).

Um den Einfluss oberflächennaher Strukturen (vgl. Kap. 1.2.1) in Form von Störkörpern auszuschließen, werden vorwiegend phasensensitive Größen betrachtet.

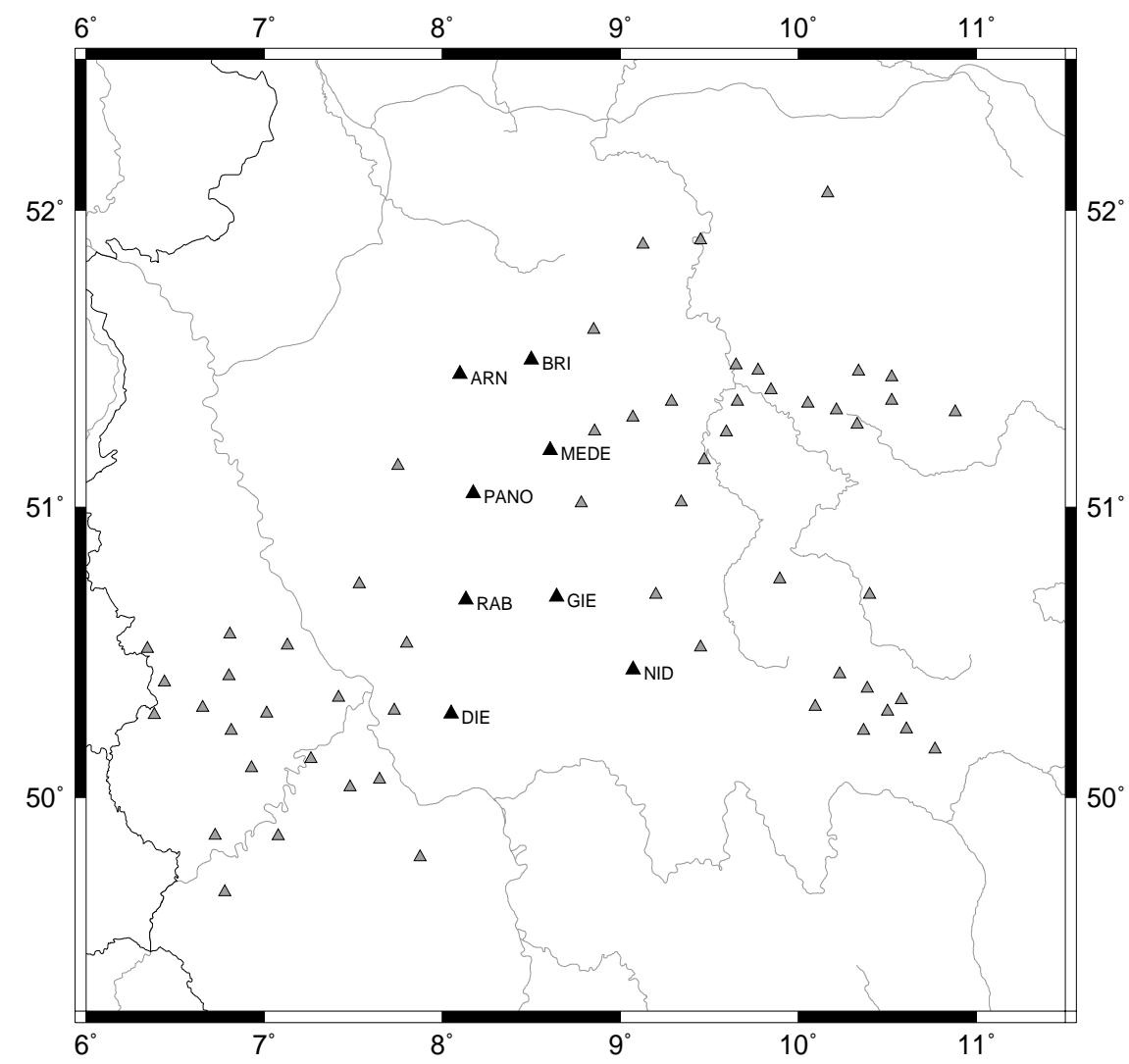

Abbildung 2.2: Karte des Messgebietes. Die mit schwarzen Dreiecken gekennzeichneten Stationen sind charakteristisch für den westlichen Teil. 

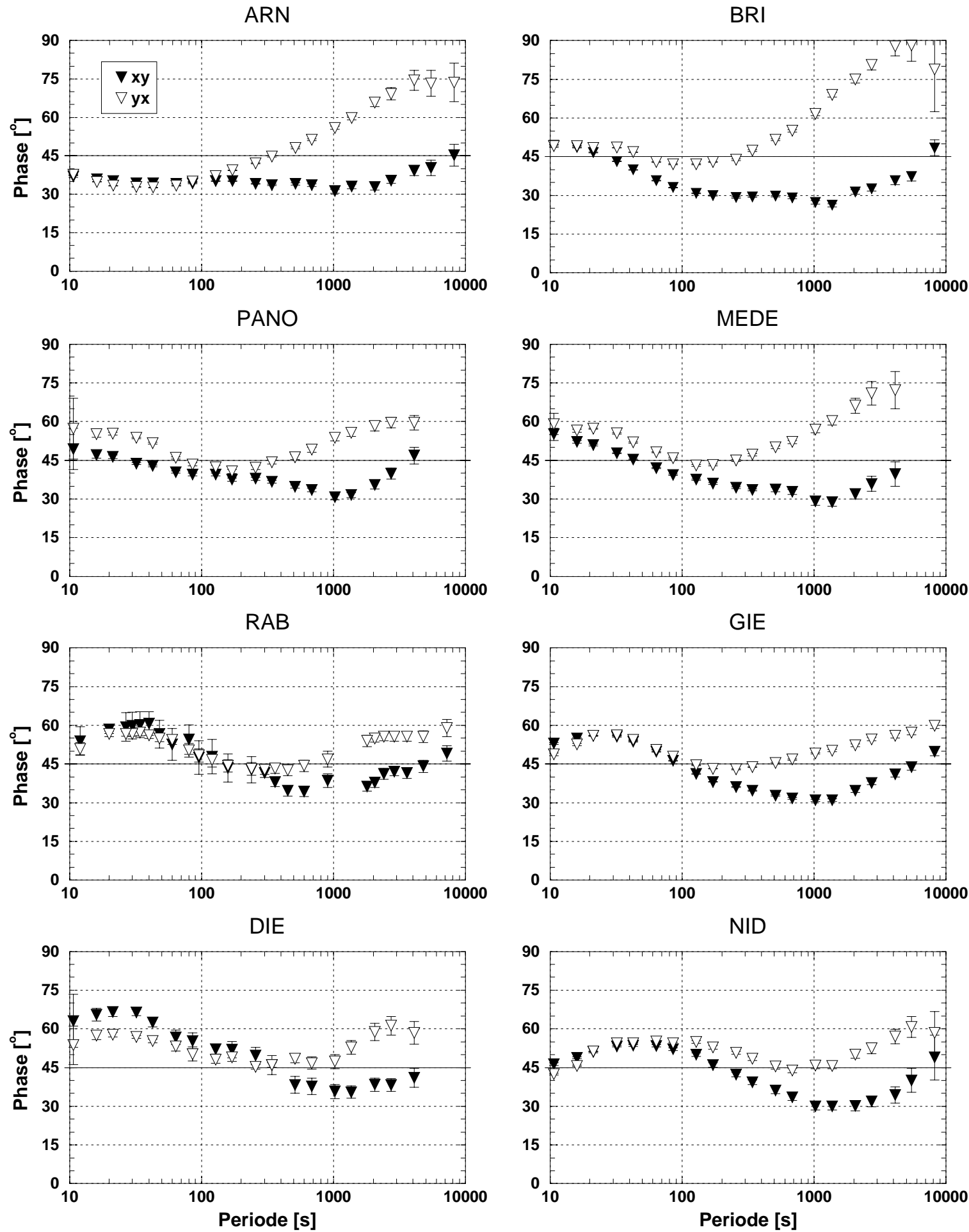

Abbildung 2.3: Unrotierte Phasenkurven der xy- und yx-Polarisation der acht charakteristischen Stationen. 


\subsubsection{Die Phase der Impedanz}

In Abb. 2.3 sind die unrotierten Phasenkurven der acht charakteristischen Stationen dargestellt (vgl. 1.5). Die Anordnung der Stationen stellt einen Nord-Süd-Schnitt durch das Messgebiet dar. Der Periodenbereich kann bei annähernd allen Stationen im westlichen Teil des Arrays in zwei charakteristische Bereiche geteilt werden, einen zwischen (I) $10 \mathrm{~s}$ und $500 \mathrm{~s}$ und einen (II) für Perioden $>500 \mathrm{~s}$. Im Bereich (I) ist festzustellen, dass die Phasenkurven beider Polarisationen für alle Stationen kein einheitliches Verhalten zeigen. Diese lateralen Variationen zwischen verschiedenen Stationen spiegeln kleinräumige Änderungen der elektrischen Leitfähigkeit in der mittleren und unteren Kruste wider. Im Bereich (II) zeigen die Phasenkurven aller Stationen ein einheitlicheres Verhalten. Die Phasen der xy-Polarisation liegen unter $45^{\circ}$, mit einem Minimum von $30^{\circ}$ bei etwa $1000 \mathrm{~s}$. Zu längeren Perioden steigen die Werte der Phase wieder an und nähern sich $45^{\circ}$. Fast alle Stationen des westlichen Abschnitts des Arrays zeigen diese identischen Phasenkurven der xy-Polarisation für lange Perioden.
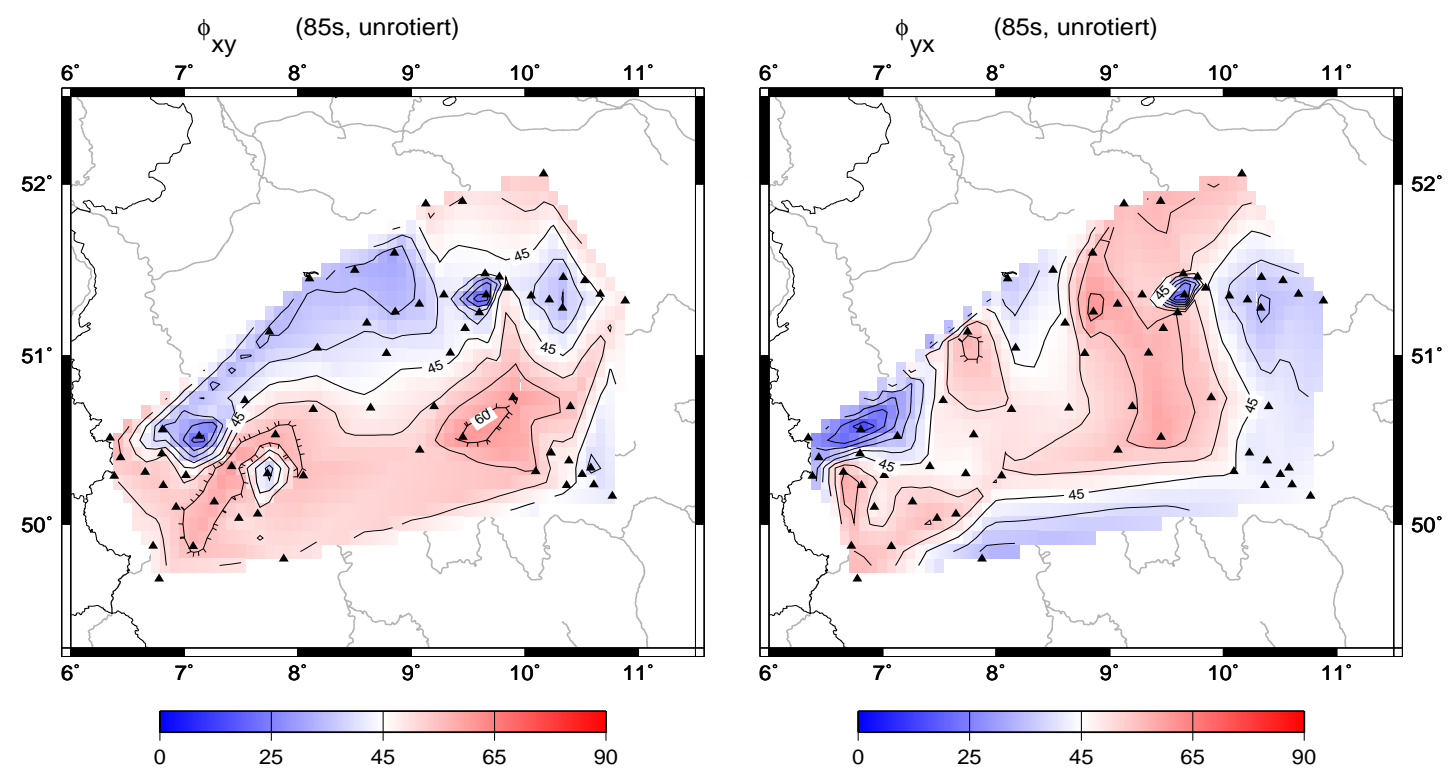

Abbildung 2.4: Flächenhafte Darstellung der unrotierten Phasen der xy- und yxPolarisation bei der Periode von $85 \mathrm{~s}$.

Die Phase der yx-Polarisation liegt demgegenüber für Perioden $>500 \mathrm{~s}$ über $45^{\circ}$ und steigt zu langen Perioden hin an. Dieser Anstieg der Phasenkurve wird bei fast allen Stationen im westlichen Bereich des Messgebiets beobachtet, mit einer Zunahme von Süden nach Norden (Abb. 2.3). Das Maximum dieser Phasenaufspaltung liegt bei ungefähr $2000 \mathrm{~s}$ und nimmt für lange Perioden wieder ab. Diese Charakteristik wird 
durch die flächenhafte ${ }^{2}$ Darstellung der Phasen beider Polarisationen verdeutlicht. In Abb. 2.4 ist für $85 \mathrm{~s}$ stellvetretend für die kürzeren Perioden (I) die unrotierte Phase beider Polarisationen flächenhaft abgebildet. Die linke Karte zeigt die Phase der xy-, die rechte die der yx-Polarisation. Für kurze Perioden ist in den Phasendaten kein einheitliches Verhalten zu beobachten. Ihre Eindringtiefen liegen mit p 30 km (T=84 s, $\rho=50 \Omega \mathrm{m}$ in Gl. 1.2) in der Größenordnung des mittleren Stationsabstandes. Die zu diesen Perioden gehörenden Induktionsräume benachbarter Stationen überschneiden sich somit nur gering, was die z.T. starken lateralen Variationen erklärt.
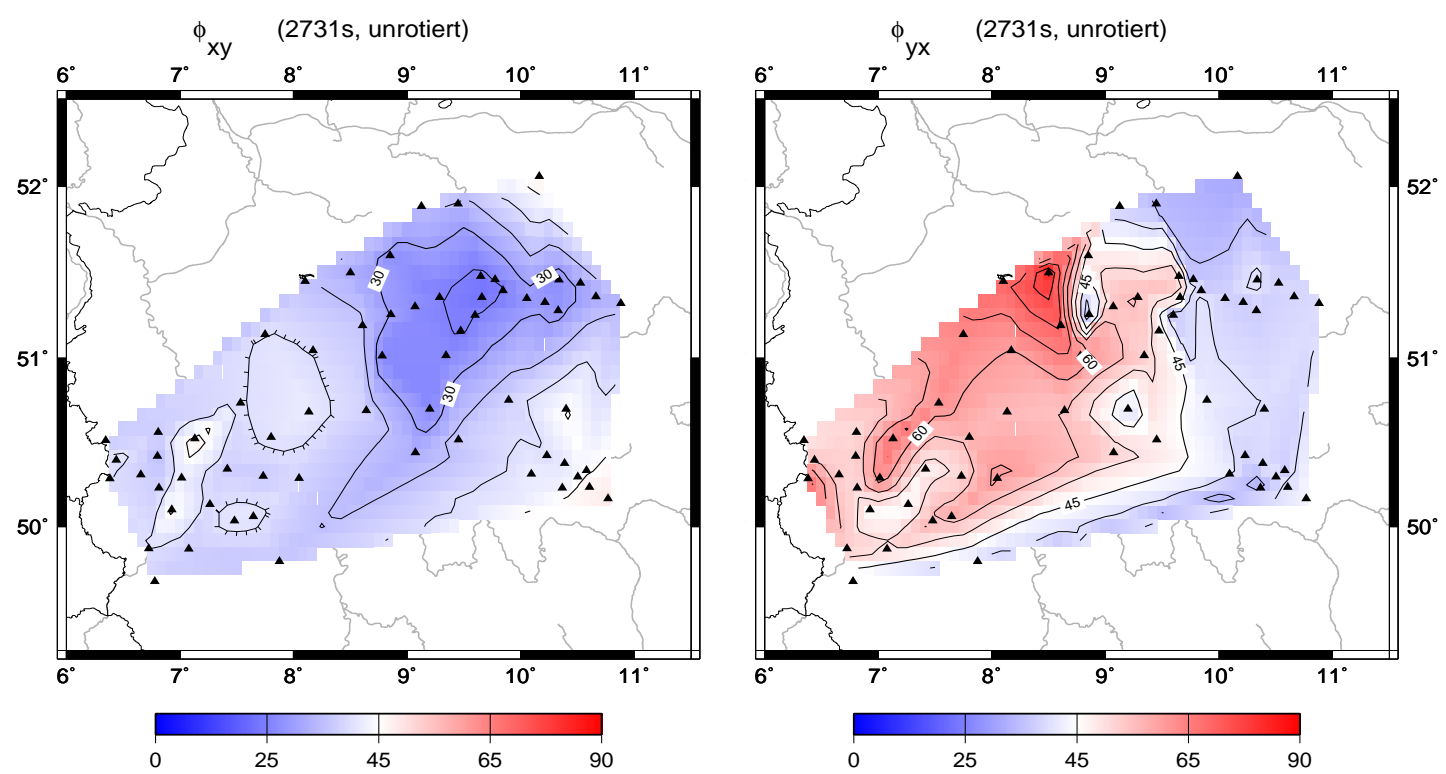

Abbildung 2.5: Flächenhafte Darstellung der unrotierten Phasen der xy- und yxPolarisation bei der Periode von 2731 s.

Der Bereich (II) ist in Abb. 2.5 anhand der Periode $2731 \mathrm{~s}$ dargestellt. Für diese langen Perioden ist der mittlere Stationsabstand im Vergleich zur Eindringtiefe $(\mathrm{p} \sim 185 \mathrm{~km})$ klein. Die Induktionsräume benachbarter Stationen sind aufgrund ihrer Überschneidung nicht mehr voneinander unabhängig. Darüber hinaus nimmt auch die Skalenlänge lateraler Ausdehnungen der elektrischen Leitfähigkeit mit der Tiefe zu. Diese beiden Effekte begründen die geringen lateralen Änderungen der Phase bei langen Perioden.

Die Phase der xy-Polarisation (Abb. 2.5, links) zeigt im gesamten Messgebiet nur wenig

\footnotetext{
${ }^{2}$ Flächenhafte Darstellungen der Daten erfolgen mit den Generic Mapping Tools (GMT) (Smith und Wessel 1990; Wessel und Smith 1991). Die Interpolation der Daten erfolgt dabei nach der Delauney Triangulation (WATSON 1982). Durch die Triangulation wird berücksichtigt, dass besonders bei langen Perioden benachbarte Stationen aufgrund der großen Eindringtiefen nicht unabhängig voneinander sind.
} 
Variationen mit Werten unter $45^{\circ}$. Die Phase der yx-Polarisation (Abb. 2.5, rechts) hingegen zeigt einen ausgedehnten Bereich hoher Phasenwerte, und lateral nur geringer Änderungen. Von Südwest (SW) nach Nordost (NO) nehmen die Phasen von $60^{\circ}$ bis auf über $75^{\circ} \mathrm{zu}$. Nach Osten ist der Bereich hoher Phasenwerte begrenzt und auch im Bereich des Rheinischen Schiefergebirges zeichnet sich nach Westen eine Verringerung der Phasenwerte ab. Aufgrund des begrenzten Arrays kann dies aber nicht mit Sicherheit festgestellt werden.

Bisher wurden die Daten lediglich unrotiert betrachtet und nur auf die Nebendiagonalelemente des Impedanztensors eingegangen. Wie in Kap. 1.2.1 gezeigt wurde, ist dies nur bei regional zweidimensionaler Struktur und vorliegenden Daten im Koordinatensystem des Streichens sinnvoll. Inwieweit dies für den vorliegenden Datensatz erfüllt ist, wird im Folgenden diskutiert.

\subsubsection{Strukturbestimmung}

Die Betrachtung der unrotierten Phase allein, ohne Kenntnis der Richtung des 2DStreichens oder angenährt abschnittsweiser 2D-Strukturen, ist wenig sinnvoll (vgl. Kap. 1.2.2). Die phasensensitiven Rotationswinkel können die zur Interpretation der Phase wichtigen Informationen über mögliche einfache Leitfähigkeitsverteilungen liefern. In Abb. 2.6 sind die phasensensitiven Rotationswinkel der acht charakteristischen Stationen über der Periode aufgetragen. Benachbarte Stationen zeigen dabei einen z.T. ähnlichen Frequenzgang des Winkels. Um die Charakteristika in den Daten stärker hervorzuheben, sind in Abb. 2.7 die Rotationswinkel dieser acht Stationen gemeinsam aufgetragen. Wie bei der Phase, so findet sich auch beim Rotationswinkel die charakteristische Zweiteilung des Periodenbereichs. Bei kurzen Perioden streuen die Winkel stark, im Mittel zwischen $20^{\circ}$ und $45^{\circ}$. Zu beobachten ist dies besonders bei der Station ARN bei kurzen Perioden $(<256$ s, vgl. Abb. 2.6). Zu langen Perioden nimmt die Streuung der Rotationswinkel ab. Insbesondere benachbarte Stationen zeigen gleiches Verhalten (NID u. GIE, DIE u. RAB od. PANO u. MEDE, Abb. 2.6). Die Winkel liegen für Perioden $>1000 \mathrm{~s}$ bei $0^{\circ} \mathrm{bzw}$. $90^{\circ 3}$.

Abb. 2.8 stellt flächenhaft die phasensensitiven Winkel für die beiden Periodenbereiche dar. Jedes Kreuz symbolisiert ein Koordinatensystem, das um den phasensensitiven Winkel einer jeden Station bei 85 s und 2731 s rotiert ist. Die Länge der Koordinatenachsen

\footnotetext{
${ }^{3}$ Aufgrund der funktionalen Form zur Berechnung des phasensensitiven Rotationswinkels ( 1.11) ergibt sich für den Winkel eine Unsicherheit von $\pm 90^{\circ}$. Es ist üblich, Winkel zwischen $0^{\circ}$ und $90^{\circ}$ anzugeben, daher werden Werte knapp außerhalb dieses Bereiches um $90^{\circ}$ nach oben oder unten verschoben. Allein anhand des Drehwinkels kann jedoch nicht entschieden werden, ob $\alpha_{B}$ senkrecht oder parallel zum 2D-Kontrast (vgl. Kap. 1.2.2) verläuft. Das die Winkel zur gleichen Streichrichtung gehören folgt aus der Betrachtung der Phasenaufspaltung (Abb. 2.8, rechts)
} 

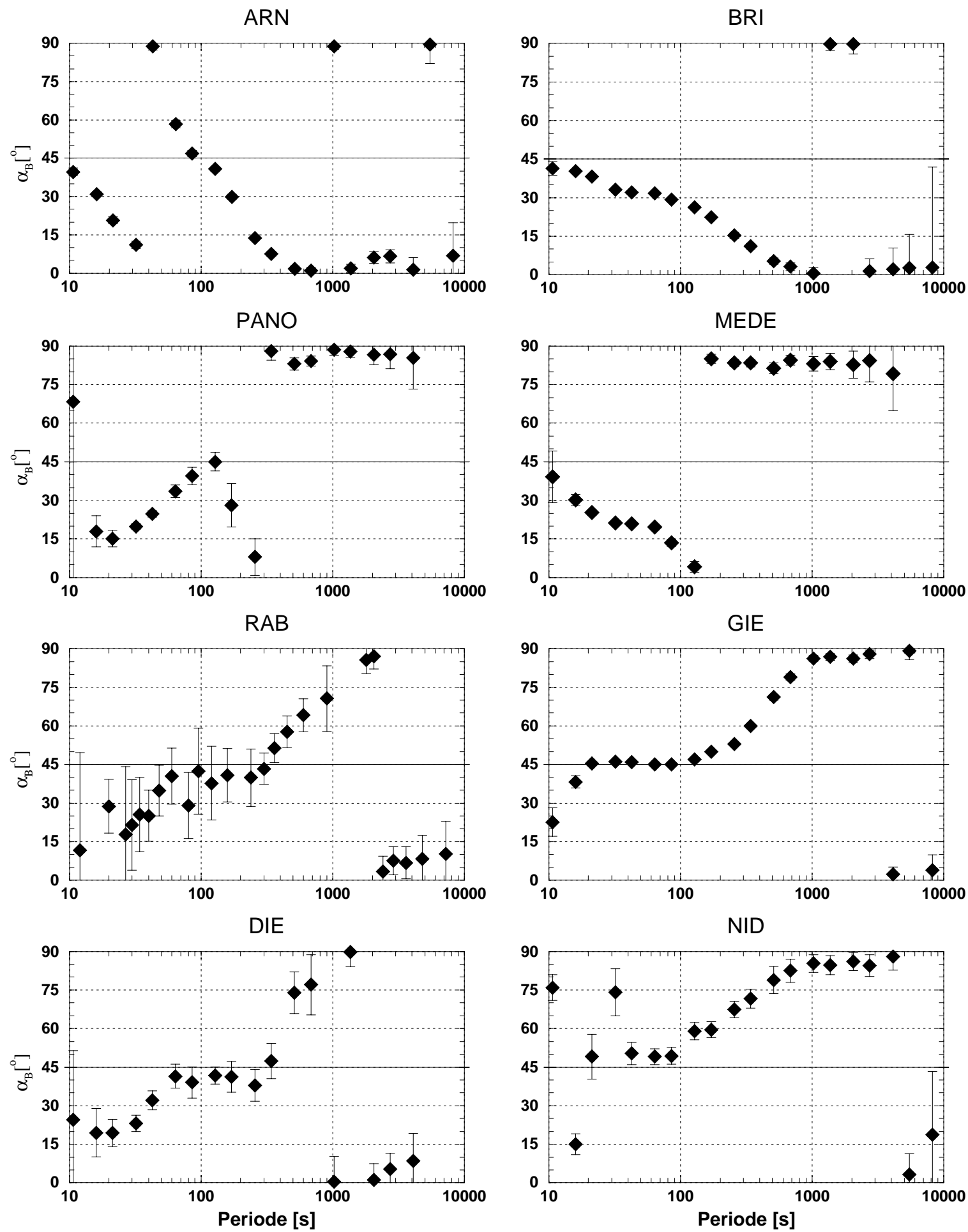

Abbildung 2.6: Frequenzgang des phasensensitiven Rotationswinkels der 8 Stationen. 


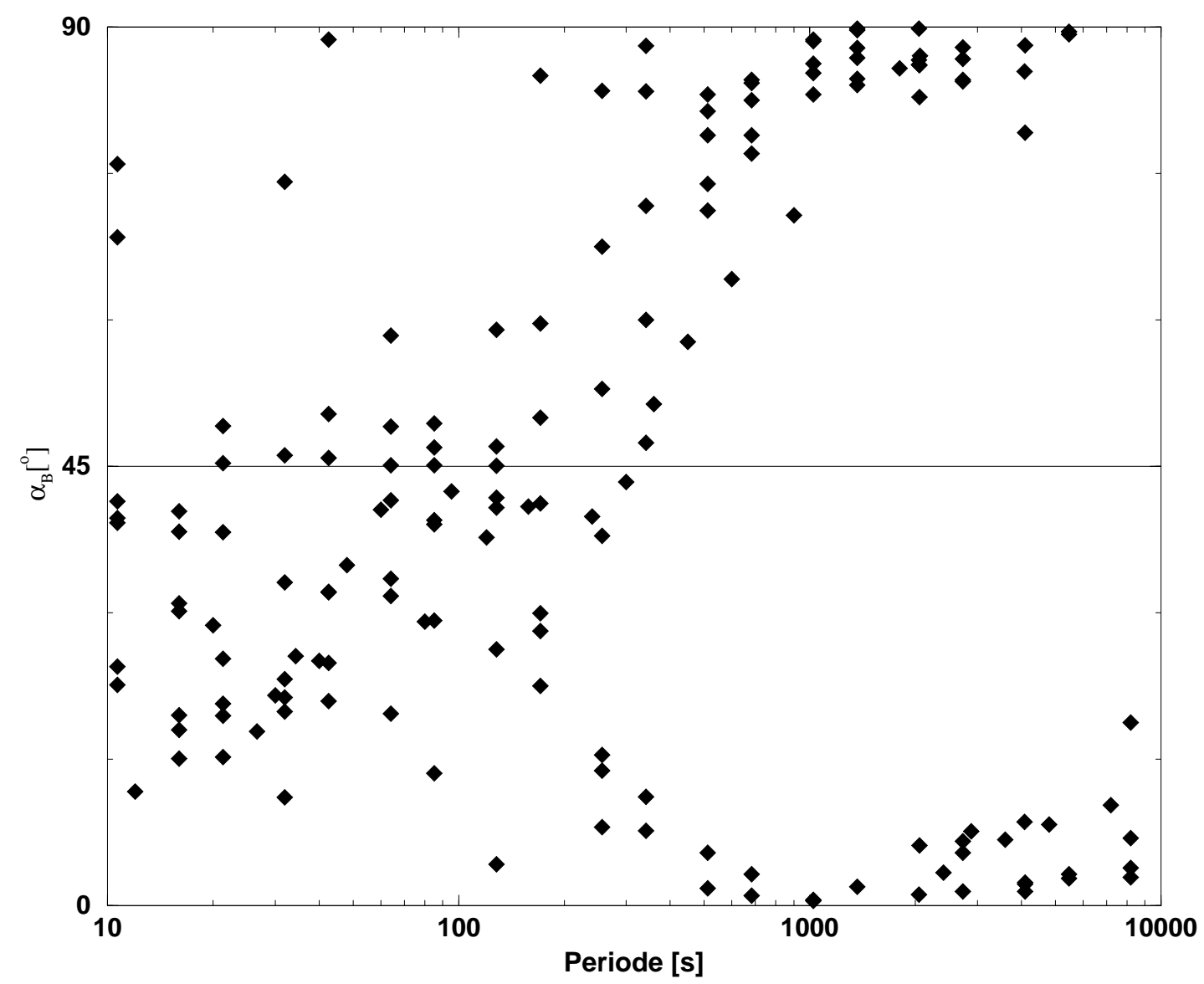

Abbildung 2.7: Phasensensitive Rotationswinkel der 8 Stationen. Um die Charakteristika hervorzuheben, sind die Frequenzgänge gemeinsam dargestellt.

ist ein Maß für die rotierte Phase in diesem Koordinatensystem. Die grauen Balken stellen die Phase der xy-Polarisation $\phi_{x y^{\prime}}$, die schwarzen die der yx-Polarisation $\phi_{y x^{\prime}}$ dar. Die kurzen Perioden sind in Abb. 2.8 (links) anhand von 85 s dargestellt. Auch bei Betrachtung des gesamten Messgebiets zeigt sich bei kurzen Perioden ein im Mittel einheitlicher Winkel um $45^{\circ}$, wobei benachbarte Stationen z.T. stark differierende Richtungen aufweisen.

Für lange Perioden (Abb. 2.8, rechts) zeigt sich ein einheitlicheres Bild. Im gesamten Messgebiet ändern sich die Rotationswinkel lateral, d.h. zwischen benachbarten Stationen nur wenig. Besonders im Bereich erhöhter Phasenwerte der yx-Polarisation (Abb. 2.5) zeigen die Winkel eine einheitliche Richtung. Die Koordinatensysteme sind Nord-Süd (NS) bzw. West-Ost (WO) ausgerichtet, was einem Rotationswinkel von $0^{\circ}$ bzw. $90^{\circ}$ entspricht.

Im Array zeigt sich für lange Perioden ein ausgedehntes Gebiet einheitlichen Verhaltens der Rotationswinkel und der Phasenkurven der xy- und yx-Polarisation. Für diese Perioden und somit für große Eindringtiefen ändert sich in diesem Gebiet die Leitfähigkeit 

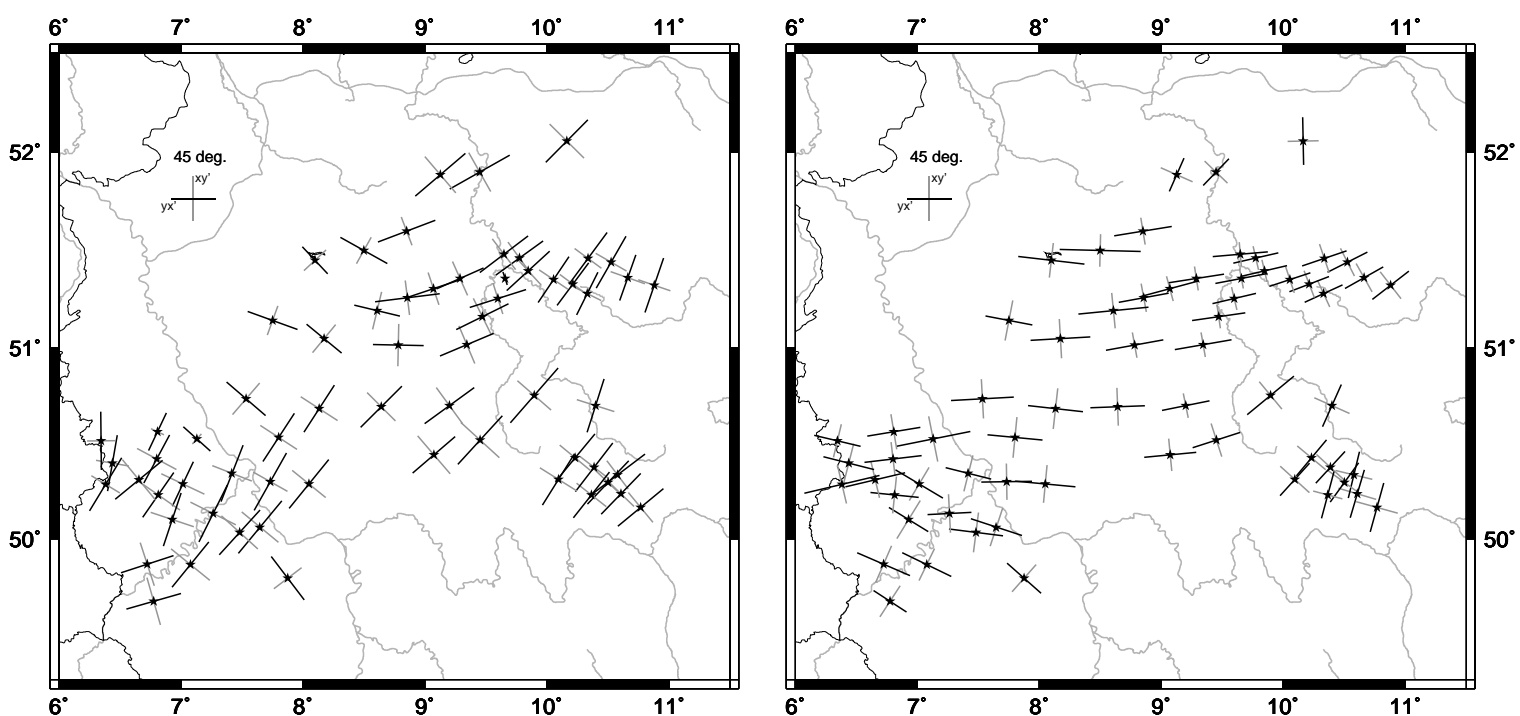

Abbildung 2.8: Phasensensitiver Rotationswinkel und rotierte Phasen der xy'- und yx'Polarisation bei 85 s (links) und 2731 s (rechts). In schwarz ist die Phase der xy- in grau die der yx-Polarisation dargestellt. Die Länge der Achsen des Referenzkreuzes entspricht $45^{\circ}$.

lateral nicht oder nur wenig. Bei gleichzeitigen Rotationswinkeln um $0^{\circ} / 90^{\circ}$ bedeutet dies, dass für lange Perioden die Daten unrotiert interpretiert werden können, das Koordinatensystem des Streichens ist annähernd NS-WO ausgerichtet.

Die beobachtete flächenhafte Phasenaufspaltung (mit $\phi_{x y}<45^{\circ}$ und $\phi_{y x}>45^{\circ}$ ) deutet, zusammen mit den in diesem Bereich einheitlichen Drehwinkeln auf eine ausgedehnte Richtungsabhängigkeit der Leitfähigkeit hin (vgl. Kap. 1.2). Dabei ist die Leitfähigkeit in WO-Richtung größer als senkrecht dazu. In Abb. 2.9 ist der Rotationswinkel gemeinsam mit der Phasenaufspaltung bei 2731 s aufgetragen. Jeder Balken gibt die aus (1.11) berechnete Richtung der genäherten 2D-Struktur und dessen Länge die Phasendifferenz in diesem Koordinatensystem an. Im westlichen Teil des Arrays zeigen nahezu alle Stationen ${ }^{4}$ ein ähnliches Verhalten, die Balken sind ungefähr in WO-Richtung ausgerichtet und zeigen Phasenaufspaltungen von $>20^{\circ}$. Die Aufspaltung der Phasenkurven nimmt zum östlichen Teil des Arrays hin ab, gleichzeitig drehen die Rotationswinkel in nördliche Richtung.

Unter Berücksichtigung der zur Darstellung der Daten gemachten Annahmen kann Abb. 2.9 genutzt werden, eine qualitative Aussage über die Leitfähigkeitsverteilung zu treffen. Die Richtung der Balken stellt die Richtung der hohen Leitfähigkeit dar, deren

\footnotetext{
${ }^{4}$ Abweichungen besonders im Rheinischen Schiefergebirge sind z.T. auf durch schlechtere Datenqualität bedingte größere Fehler besonders bei den langen Perioden zurückzuführen.
} 


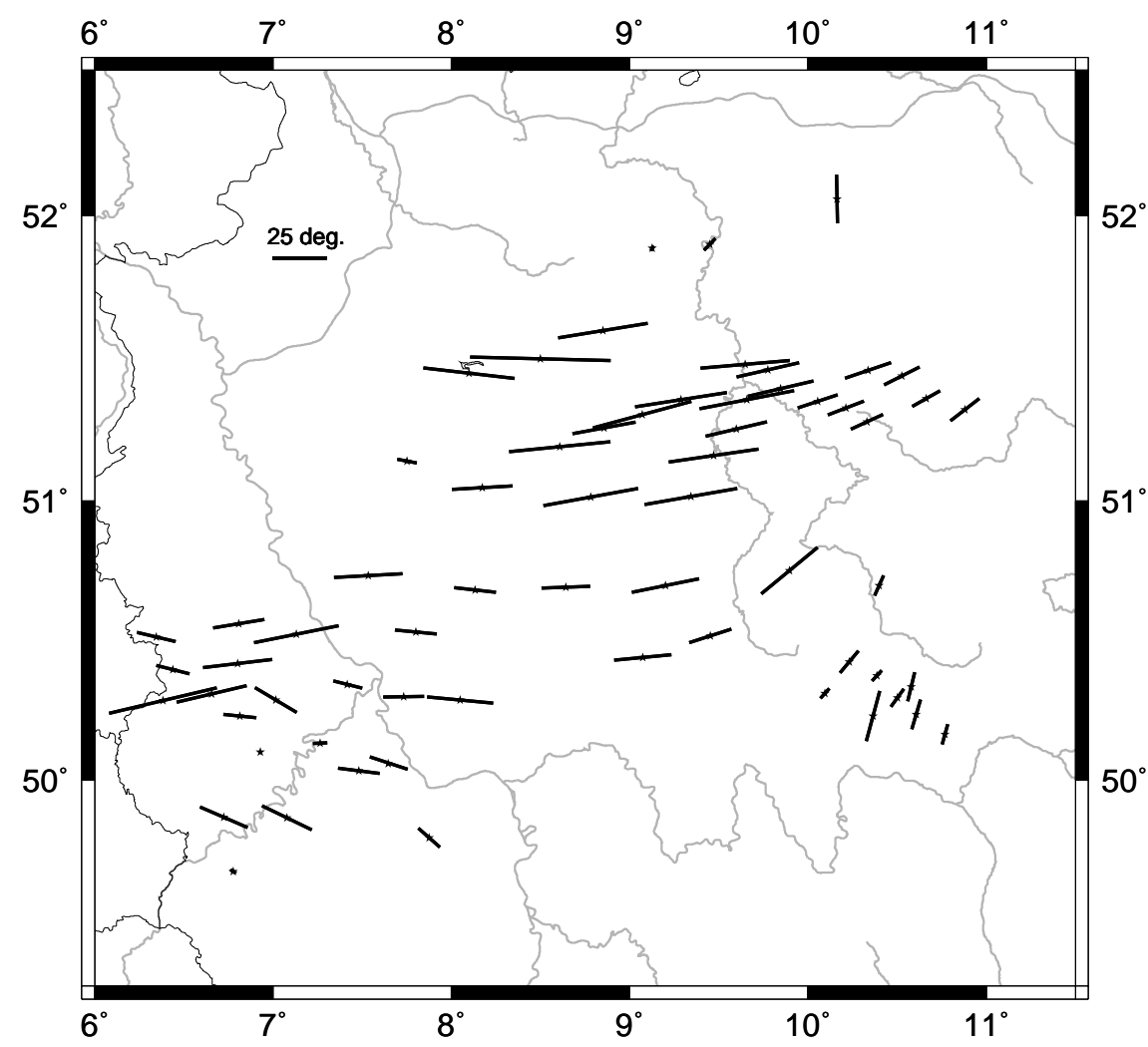

Abbildung 2.9: Differenz der rotierten Phasen beider Polarisationen bei 2731 s. Jede Station ist rotiert um den phasensensitiven Winkel bei dieser Periode. Der Länge des Referenzbalkens entspricht eine Phasenaufspaltung von $25^{\circ}$.

Länge ein Maß für die Anisotropie ist (Kellett et al. 1992; MAReschal et al. 1995; JI et al. 1996). Neben räumlicher Invarianz als Rechtfertigung für ,abschnittsweise 2D-Interpretation“ kann zusätzlich die Erfüllung des Kriteriums zur Berechnung des Rotationswinkels $\alpha_{B}$ (1.11) überprüft werden. In Abb. 2.10 sind die Phasen der vier Tensorelemente für lange Perioden $(T>1000 \mathrm{~s})$ beispielhaft anhand der Station PANO für alle Stationen im Bereich der Phasenaufspaltung dargestellt. Zur besseren Übersichtlichkeit wird auf eine Darstellung der Fehler verzichtet. Da für lange Perioden ( $>1000 \mathrm{~s}$ ) der phasensensitive Winkel stabil ist (vgl. Abb. 2.6), ist der Tensor für jede Periode um den phasensensitiven Winkel bei 2731 s rotiert. Die gute Übereinstimmung der Phasen $\phi_{x x}$ und $\phi_{y x}$, sowie $\phi_{x y}$ und $\phi_{y y}$ im Rahmen des Fehlers macht deutlich, dass auch analytisch die Bedingung zur Anwendung von (1.11) erfüllt ist (vgl. Kap. 1.2.2).

Durch die bisherige Betrachtung der Daten konnte die regionale, lange Perioden beeinflussende Leitfähigkeitsstruktur, als genähert anisotrop interpretiert werden. Die $\rho^{*}$ $\mathrm{z}^{*}$-Darstellung kann daher, um einen ersten Eindruck über die Tiefenverteilung der 


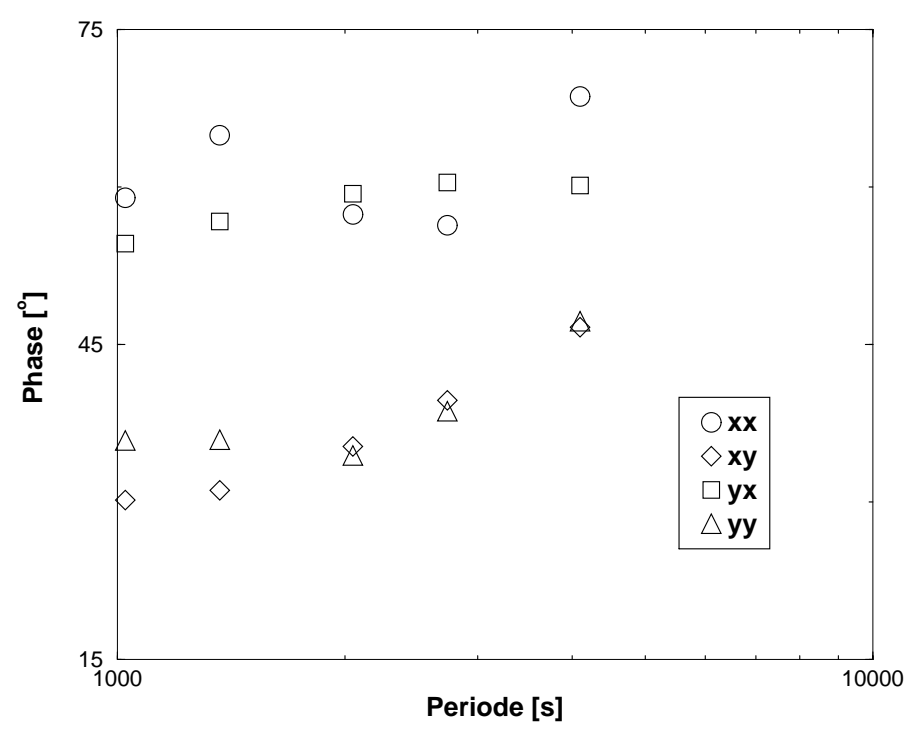

Abbildung 2.10: Phasen der vier Elemente des Impedanztensors im Periodenbereich $10^{3}-10^{4} \mathrm{~s}$ der Station PANO, rotiert um den phasensensitiven Winkel der Periode $2731 \mathrm{~s}$.

Leitfähigkeit entlang der beiden Polarisationen zu gewinnen, verwendet werden. Diese Leitfähigkeits-Tiefenauftragung ist, durch den Einfluss von oberflächennahen Störkörpern in die Darstellung, als rein qualitativ anzusehen. In Abb. 2.11 ist dies für zwei Stationen im Bereich großer Phasenaufspaltung dargestellt. Für die xy-Polarisation, sowohl bei Station ARN als auch MEDE nimmt mit zunehmender Periode und Eindringtiefe der spezifische Widerstand $\rho^{*}$ zu. Laufen für kurze Perioden die Kurven von xy und yx zunächst noch parallel, nimmt zu längeren Perioden der spezifische Widerstand der yx-Polarisation stark ab. Die yx- ist im Gegensatz zur xy-Polarisation von einem guten Leiter beeinflusst.

Dies zeigt sich insbesondere bei Betrachtung der Eindringtiefen $z^{*}$, die für die yxPolarisation bei Perioden $>1000$ s nur gering oder nicht zunehmen. Die längsten der gemessenen Perioden durchdringen den guten Leiter dabei nicht.

Der Vergleich der beiden Stationen in Abb. 2.11 macht darüber hinaus den Einfluss lokaler Störkörper deutlich. Für die Periode 2731 s variieren die Eindringtiefen $z^{*}$ der yxPolarisation der beiden Stationen um mehr als $400 \mathrm{~km}$. Bei einem Stationsabstand von ungefähr $50 \mathrm{~km}$ kann diese große Differenz nicht nur auf induktiv wirkende Leitfähigkeitsstrukturen zurückgeführt werden. In die Berechnung sowohl von $\rho^{*}$ als auch $z^{*}$ (vgl. 1.6) geht die Amplitude der Impedanz mit ein. Da diese von Verzerrung beeinflusst ist, sind die unterschiedlichen Eindringtiefen mehr statischen als induktiven Effekten zuzuschreiben. Dieses einfache Beispiel verdeutlicht, dass eine quantitative Auswertung der Amplitude ohne Entzerrung (Kap. 1.2.2) nicht sinnvoll ist. 

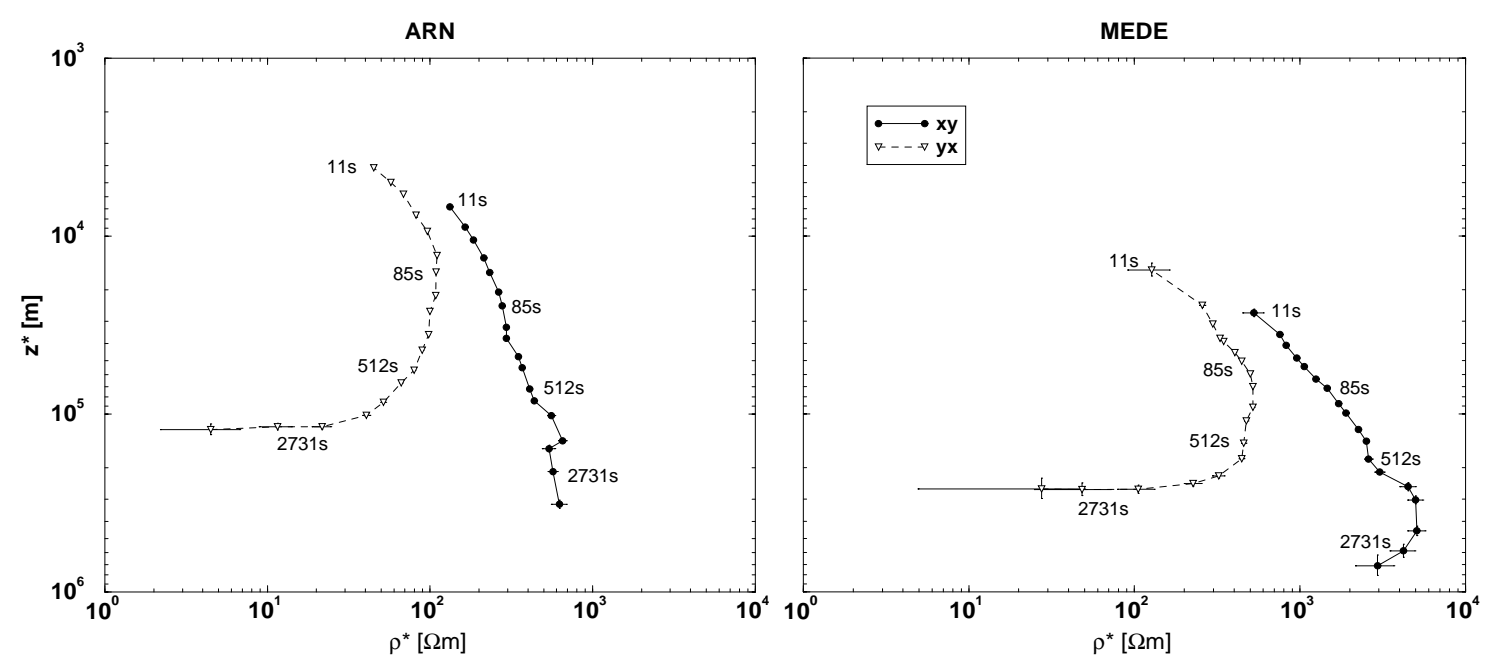

Abbildung 2.11: $\rho^{*}-z^{*}$-Darstellung der unrotierten Daten zweier Stationen im nördlichen Teil des Arrays.

\subsubsection{Lokale Induktionspfeile}

In Abb. 2.12 sind die lokalen Induktionspfeile (1.13) für $2731 \mathrm{~s}$ dargestellt, wobei die schwarzen Pfeile die Real- und die grauen die Imaginärpfeile abbilden. Im nördlichen Abschnitt des Messgebietes ist bei langen Perioden ein starkes vertikales Magnetfeld zu beobachten, wie in der Abbildung anhand der langen, in südwestliche Richtung weisenden Induktionspfeile (Länge der Realpfeile > 0.5) dargestellt. Ursache dieses starken vertikalen Magnetfelds ist der Leitfähigkeitskontrast am südlichen Rand der Norddeutschen Leitfähigkeitsanomalie. Diese Anomalie, die auf in der Norddeutschen Tiefebene gelegenen gutleitfähigen Sedimenten beruht, ist durch JUNGE (1993) als WO-streichende 2D-Struktur beschrieben worden.

Als zweite große Anomalie hat die Göttinger D-Anomalie im nördlichen Teil des Messgebietes auf die Daten Einfluss (PETER 1994; SCHNEIDER 1998). Für induktiv nicht gekoppelte Körper überlagern sich die Induktionspfeile jeder einzelnen Anomalie näherungsweise additiv (WEAVER 1990; SIEMON 1991). Für den südlichen Bereich Göttingens können die in südöstliche bzw. -westliche Richtung weisenden Pfeile somit vorwiegend als Überlagerung von Norddeutscher Leitfähigkeitsanomalie und D-Anomalie gesehen werden (SCHNEIDER 1998).

Für die Betrachtungen dieser Arbeit ist jedoch die generelle Abnahme der Länge der Induktionspfeile gegen Südwesten - bei Beibehaltung der südwestlichen Richtung - interessanter. Aufgrund der von Norden nach Süden abnehmenden Länge der Induktionspfeile scheint die Leitfähigkeitsverteilung im Bereich der Phasenaufspaltung bei langen Perioden auf sie keinen Einfluß zu haben. Dies spricht gegen eine reine 2D- und für eine ani- 
sotrope Leitfähigkeitsverteilung als Ursache der Phasenaufspaltung, da ein 2D-Kontrast Ursache eines zusätzlichen vertikalen Magnetfeldes wäre.

Im Bereich des Rheinischen Schiefergebirges nimmt die Länge der Induktionspfeile von Ost nach West wieder zu. Ursache dieser Zunahme im westlichen Teil ist eine oberflächennahe, sehr leitfähige Anomalie (LEIBECKER 2000), deren Ausdehnung und sehr hohe Leitfähigkeit sich bis zu langen Perioden auswirkt.

Obwohl diese einfache Betrachtung sicherlich nicht umfassend ist, wird das Fehlen des Einflusses auf die Induktionspfeile als weiteres Indiz für eine anisotrope Leitfähigkeitsstruktur gesehen.

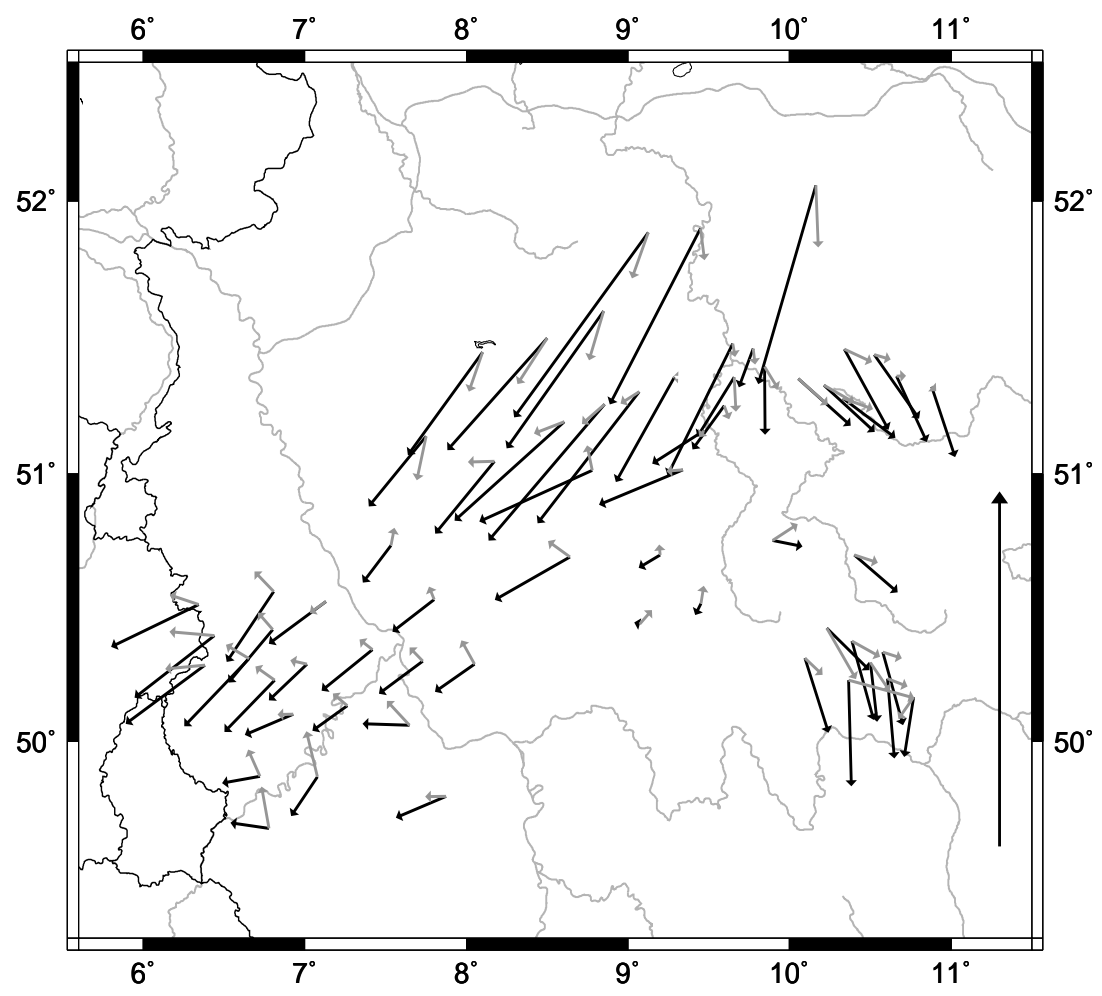

Abbildung 2.12: Flächenhafte Darstellung der lokalen Induktionspfeile bei der Periode von 2731 s. Realpfeile sind in schwarz, Imaginärpfeile in grau dargestellt. Der am rechten Rand eingezeichnete Referenzpfeil entspricht der Länge eins. 


\section{Ein Modell der Leitfähigkeitsverteilung}

Die an der Erdoberfläche gemessenen Daten und die darin enthaltenen Informationen über die Leitfähigkeitsverteilung im Untergrund sollen durch ein Leitfähigkeitsmodell erklärt werden. Hierfür sind prinzipiell zwei Ansätze denkbar: zum einen die Inversion eines Teils oder des gesamten Datensatzes, zum anderen die Anpassung eines oder mehrerer Parameter durch Vorwärtsmodellierung.

Für die Interpretation von Profildaten und dementsprechend zweidimensionaler Fragestellungen stellt die Inversion ein vielfach benutztes Verfahren zur Anpassung der Messdaten dar. Dabei wird aus dem unendlich-dimensionalen Lösungsraum des Gleichungssystems versucht, ein minimum structure model $^{1}$ zu finden, welches im Rahmen der Messfehler die Daten am besten erklärt (DE GROOT-Hedlin und CONSTABLE 1990).

Zur Lösung dreidimensionaler Probleme wird die Vorwärtsmodellierung der Inversion vorgezogen. Die wenigen existierenden Inversionscodes sind zum Teil kommerziell und noch nicht frei verfügbar (NEWMAN und ALUMBAUGH 2000; ZHDANOV et al. 2000), weiterhin erfordern sie eine enge, gleichmäßige Stationsüberdeckung (G. Newman, pers. Mitteilung), wie sie in Feldmessungen meist nicht zu realisieren ist. Die Lösung der zum Teil stark unterbesetzten Gleichungssysteme lässt dann keine sinnvolle Interpretation der Daten zu.

Bei der dreidimensionalen Vorwärtsmodellierung wird hingegen versucht, durch möglichst einfache Leitfähigkeitsmodelle einzelne Parameter in den Daten anzupassen. Durch sorgfältige Analyse der Messdaten und unter Berücksichtigung von Voruntersuchungen wird durch zahlreiche Vorwärtsmodellierungen die Abweichung zwischen Daten und Modellantwort minimiert.

Zur Modellierung der Daten wird in dieser Arbeit der Code von MACKIE und BoOKER (1999) verwendet, der eine modifizierte Version des 3D-Finite Differenzen Algorithmus von MACKIE et al. darstellt (MACKIE und MADDEN 1993; MACKIE et al. 1993; MACKIE et al. 1994). Auf die zum Verständnis des in Kap. 3.1.2 beschriebenen Modells

\footnotetext{
${ }^{1}$ Dieses, auf den englischen Philosoph, Logiker und Franziskanermönch William von Ockham zurückgehende Prinzip sagt, dass von allen möglichen Lösungen die einfachste gewählt werden sollte.
} 
notwendigen Besonderheiten des Programms wird im Anhang A.2.2 eingegangen.

\subsection{Ein einfaches Leitfähigkeitsmodell für das Messgebiet}

In diesem Kapitel wird ein dreidimensionales Leitfähigkeitsmodell als Ergebnis von Vorwärtsmodellierungen der in Kap. 2 vorgestellten Daten präsentiert. Mit diesem Modell werden die dort diskutierten Charakteristika in den Daten erklärt.

\subsubsection{Vorüberlegungen: 1D-Modell}

Einen ersten Eindruck über Tiefenlage sowie vorhandene Leitfähigkeiten kann durch 1DInversion der unrotierten $\rho_{a^{-}}$und Phasenkurven der xy- und yx-Polarisation gewonnen werden. Trotz der in Kapitel 1.2.2 beschriebenen Probleme, die bei Interpretation (und somit auch Inversion) der Amplituden des Impedanztensors auftreten können, liefert die 1D-Inversion zumindest qualitativ einen Eindruck des Leitfähigkeitsverhaltens mit der Tiefe und des Verhältnisses der Leitfähigkeiten beider Polarisationen zueinander.

In Abb. 3.1 ist für die Station ARN, die sehr deutlich den Effekt der Phasenaufspaltung bei langen Perioden zeigt, das Ergebnis der 1D-Inversion dargestellt, wobei jede der beiden Polarisationen getrennt durch ein 1D-Modell angepasst wurde. Zur Anwendung kam dabei der von SCHMUCKER (1974) beschriebene $\Psi$-Algorithmus. Im linken Teil der Abbildung sind $\rho_{a}$ - und Phasenkurven der Daten und Modelle dargestellt, im rechten Teil die beiden 1D-Leitfähigkeitsmodelle. Die Diskrepanz der $\rho_{a}$-Werte der xy-Polarisation zwischen Daten und Modell beruht auf dem in Kap. 1.2.1 beschriebenen static shift-Effekt, verursacht durch oberflächennahe Inhomogenitäten, die durch ein 1D-Modell nicht anzupassen sind. Von diesem Effekt abgesehen werden $\rho_{a^{-}}$und Phasenkurven durch die 1D-Modelle gut angepasst.

Aufgrund des frequenzabhängigen phasensensitiven Winkels können bei der 1DInversion der unrotierten Daten nur die langen Perioden qualitativ interpretiert werden. Deutlich zu sehen ist die starke Richtungsabhängigkeit der Leitfähigkeit. Für die xyPolarisation zeigt das Inversionsmodell bis zu einer Tiefe von über $200 \mathrm{~km}$ eine Abnahme der elektrischen Leitfähigkeit von 40 bis auf $500 \Omega \mathrm{m}$. Für die yx-Polarisation sind bis $80 \mathrm{~km}$ Tiefe identische Leitfähigkeitswerte zum Modell der xy-Polarisation zu beobachten. Im Tiefenbereich 80 bis $150 \mathrm{~km}$ ist die Leitfähigkeit hingegen deutlich erhöht. Mit Werten von $20 \Omega \mathrm{m}$ zwischen $80-140 \mathrm{~km}$ und $0.5 \Omega \mathrm{m}$ zwischen 140 und $150 \mathrm{~km}$ Tiefe ist die integrierte Leitfähigkeit ${ }^{2}$, die zur Erhöhung der Phase für yx- und somit zur

\footnotetext{
${ }^{2}$ Die integrierte Leitfähigkeit ist das Produkt aus Leitfähigkeit und Schichtmächtigkeit einer eindimensionalen Schicht, ihre Einheit ist Siemens (S). Der Begriff der integrierten Leitfähigkeit hat besondere Bedeutung in der 1D Interpretation. Schichten gleicher integrierter Leitfähigkeit liefern identische Modellantworten.
} 
Aufspaltung der beiden Polarisationen führt, 23.000 S. Im gleichen Tiefenbereich weist die integrierte Leitfähigkeit des Modells der xy-Polarisation (mit $250 \Omega \mathrm{m}$ zwischen 80 und $140 \mathrm{~km}$ und $500 \Omega \mathrm{m}$ zwischen 140 und $240 \mathrm{~km}$ ) lediglich $260 \mathrm{~S}$ aus. Die integrierte Leitfähigkeit unterscheidet sich für die beiden Polarisationen somit um nahezu zwei Größenordnungen. Diese in den integrierten Leitfähigkeiten beobachtete Anisotropie kann aber aufgrund nicht identischer Schichtmächtigkeiten nicht direkt in Anisotropie der elektrischen Leitfähigkeit übertragen werden, liefert jedoch einen ersten Hinweis auf deren Verhältnis.

Obwohl die Stationsdichte ausreichen würde, mehrere Profile durch das Messgebiet zu legen, ist aufgrund der in Kap. 2.3.2 beschriebenen Frequenzabhängigkeit des Drehwinkels der Versuch einer 2D-Inversion wenig sinnvoll.

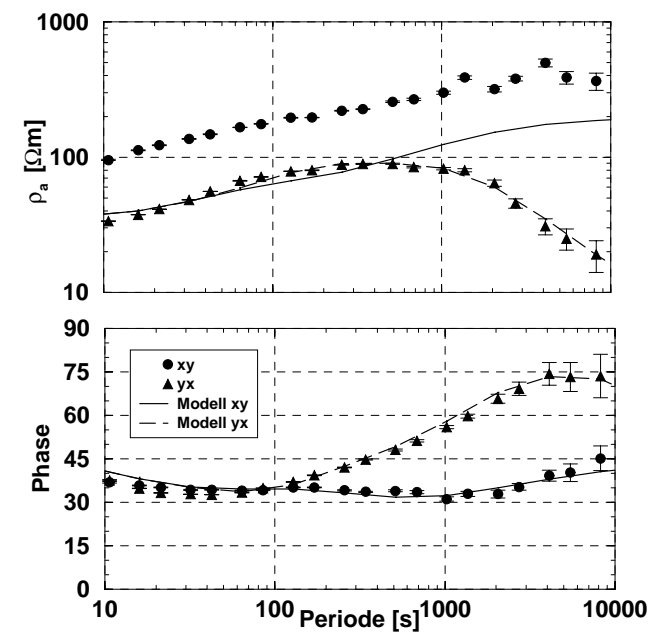

a) Unrotierte Daten mit Modellanpassung

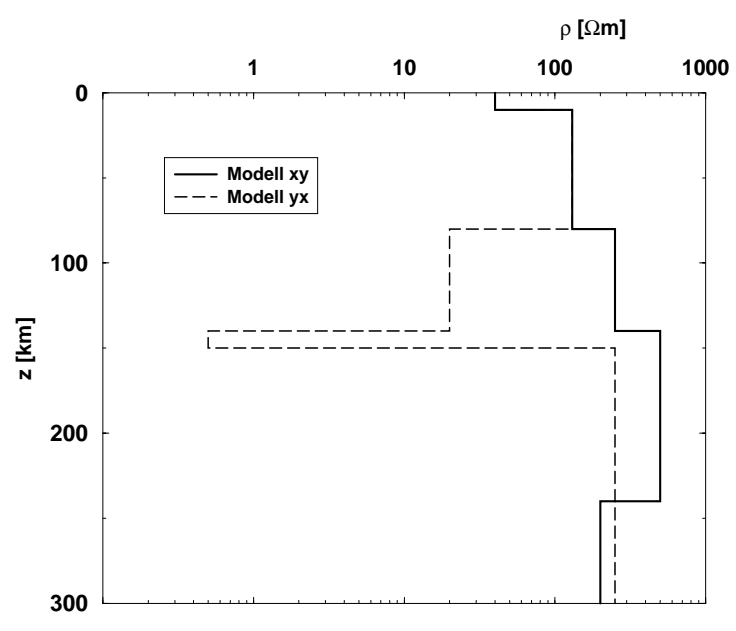

b) 1D Modelle für die beiden Polarisationen

Abbildung 3.1: Anpassung der unrotierten Daten der Station ARN. Die beiden Polarisationen werden durch je ein 1D-Modell angepasst.

\subsubsection{D-Leitfähigkeitsmodell}

Im Folgenden werden die Ergebnisse der Vorwärtsmodellierungen, die Parameter des verwendeten Grids und die Leitfähigkeitsverteilung im 3D-Modell diskutiert ${ }^{3}$. Zur besseren Übersichtlichkeit sind die Horizontal- und Vertikalschnitte durch das Grid- bzw. Leitfähigkeitsmodell dargestellt.

Die dreidimensionale Modellierung des $300 \times 400$ Quadratkilometer großen Arrays er-

\footnotetext{
${ }^{3}$ Bei der Anpassung durch Vorwärtsmodellierung handelt es sich immer um einen iterativen Prozess. Inwieweit sich durch zahlreiche Modellierungen und Modelländerungen sukzessive einem Endmodell genähert wird, zeigt der Vergleich mit GATZEMEIER (2000).
} 
folgt über ein aus $103 \times 119 \times 34$ Zellen bestehendes Grid. In Abb. 3.2 sind ein Horizontalund Vertikalschnitt durch das Modellgrid dargestellt.

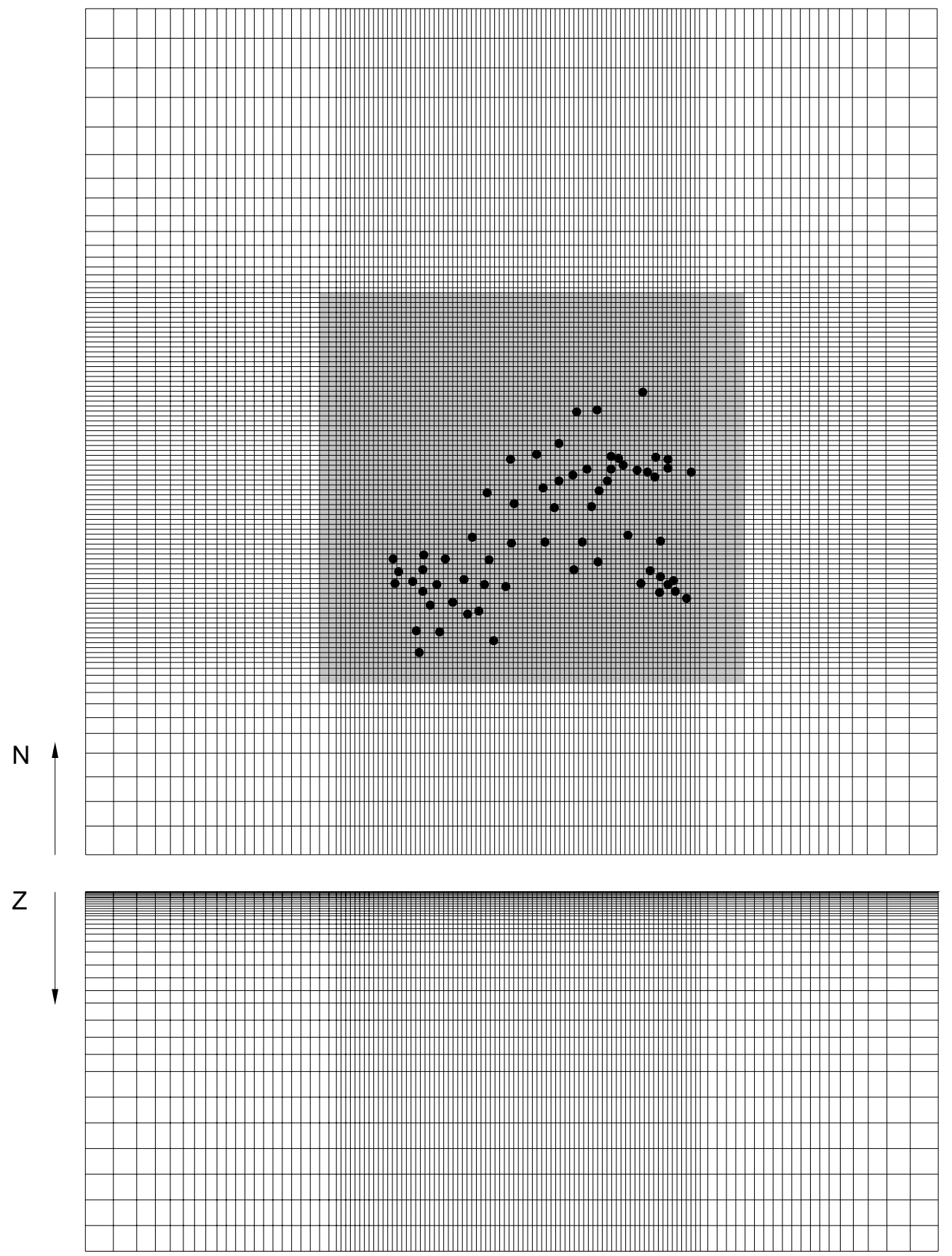

Abbildung 3.2: Oben: Aufsicht auf das Grid. Die schwarzen Punkte kennzeichnen die Lokationen der Stationen im Modellgrid. Unten:Vertikaler Ost-WestSchnitt durch das Grid. 
Die horizontale Abdeckung beträgt dabei $858 \times 923 \mathrm{~km}^{2}$, vertikal erstreckt es sich bis in eine Tiefe von $420 \mathrm{~km}$. Das Modell ist in einen inneren 3D-Kern und einen äußeren Bereich gegliedert. Der innere Bereich beinhaltet die modellierten Leitfähigkeitsstrukturen und besteht aus $5 \times 5 \mathrm{~km}^{2}$ großen Gridzellen. Im äußeren Bereich setzt sich in horizontale Richtung das 1D-Hintergrundmodell fort. Die Ausdehnung wurde so gewählt, dass sich Randeffekte des Modells an den anzupassenden Stationen nicht mehr auswirken.

Das innere, grau markierte Quadrat in Abb. 3.2 beinhaltet die im folgenden näher diskutierten Bereiche dreidimensionaler Leitfähigkeitsverteilung.

\section{D Hintergrundmodell}

Grundlage des 3D-Modells ist ein eindimensionales Hintergrundmodell, das sich aus den Ergebnissen früherer elektromagnetischer Messungen zusammensetzt (BAHR und BRUTON 1994; OetTINGER 1994; LEIBECKER 2000). Zur Skalierung der Leitfähigkeiten im Modell wählte LEIBECKER (2000) die Inversion der effektiven Impedanz $Z_{e f f}=\left(Z_{x y}-Z_{y x}\right) / 2$ (BERDICHEVSKY und DMITRIEV 1976), die den Effekt der Verzerrung in guter Näherung entfernt. Die durch Mittelung über mehrere Stationen für die Kruste erhaltenen Leitfähigkeitswerte werden für das in dieser Arbeit verwendete Array übernommen. Seine Leitfähigkeiten an der Oberfläche stimmen mit denen geoelektrischer Messungen im betrachteten Gebiet überein (GATZEMEIER 1999).

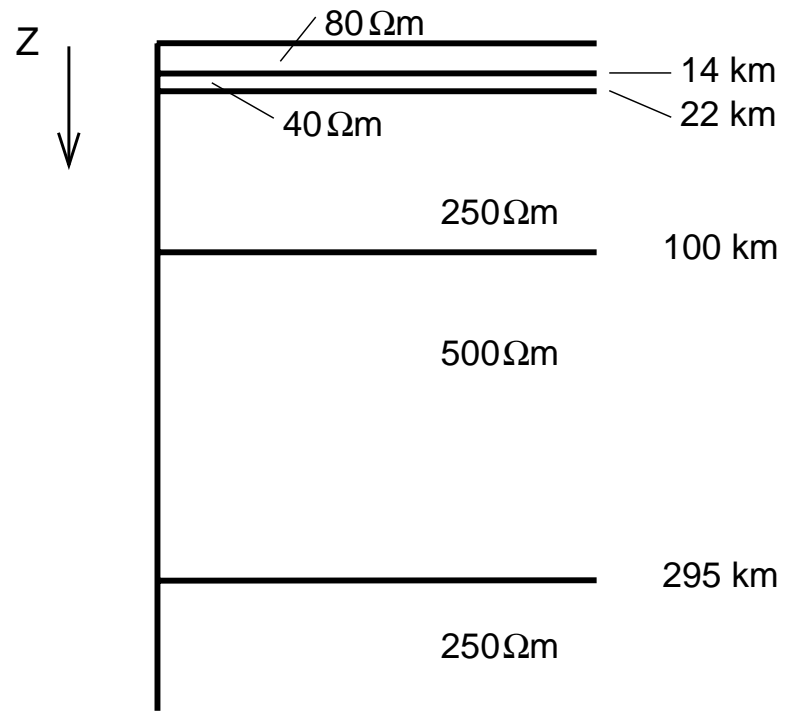

Abbildung 3.3: Vertikalschnitt durch das 1D-Hintergrundmodell.

Der Wahl des Hintergrundmodells und dessen Leitfähigkeiten kommt bei der Interpretation nur phasensensitiver Größen eine besondere Bedeutung zu, da ohne Berücksichtigung der Amplituden die absoluten Werte der Leitfähigkeiten nicht zu bestimmen sind. Durch 
die Phasenanpassung werden nur relative Änderungen der Leitfähigkeit mit der Tiefe aufgelöst.

In Abb. 3.3 ist ein schematischer Schnitt durch das 1D-Hintergrundmodell dargestellt.

\section{Obere bis mittlere Kruste}

Eingebettet in dieses Hintergrundmodell sind Leitfähigkeitsstrukturen zur Modellierung der oberen bis mittleren Kruste (Abb. 3.4), die Arbeiten entstammen, die in Teilen des Messgebietes oder angrenzenden Bereichen elektromagnetische Untersuchungen durchgeführt bzw. ausgewertet haben. Als wichtigste Strukturen sind die Norddeutsche Leitfähigkeitsanomalie (JUNGE 1993; SCHNEIDER 1998), die Krustenanomalie in der Eifel (LEIBECKER 2000) sowie die gutleitenden Sedimente des Norddeutschen Beckens (LOSECKE et al. 1978; LOSECKE et al. 1979) eingefügt. Obwohl nicht alle dieser Anomalien mit dem Array aufzulösen sind, werden sie als a-priori Informationen verwendet, um ihren Effekt in den Messdaten nicht fehlzuinterpretieren.

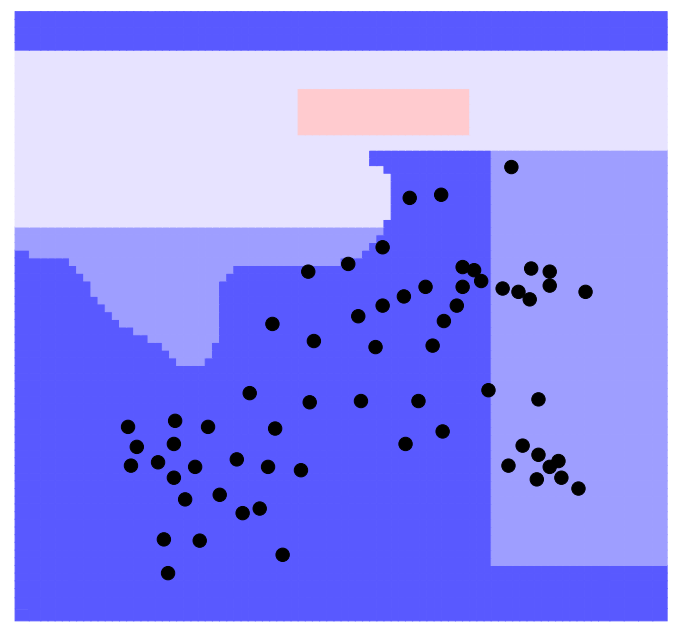

a) Strukturen in $1-5.4 \mathrm{~km}$ Tiefe.

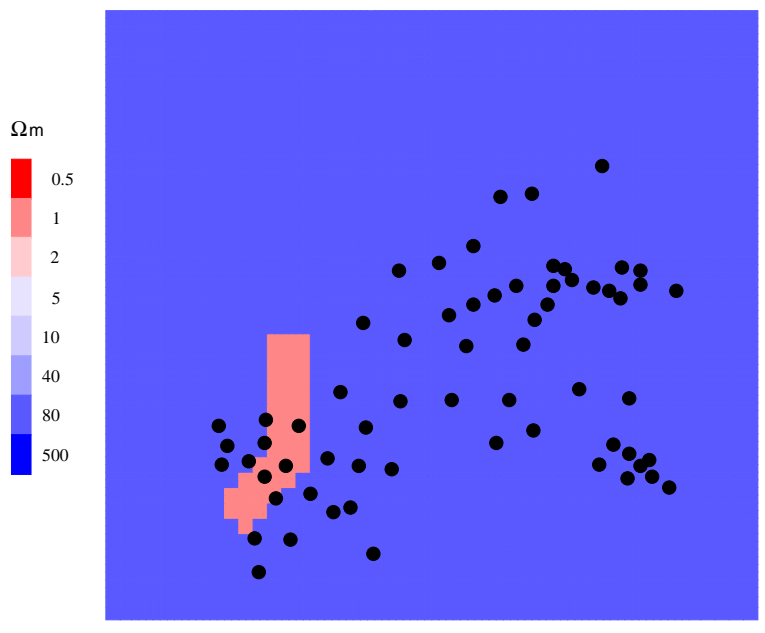

b) Krustenanomalie in der Eifel (5.4-7.2 km Tiefe).

Abbildung 3.4: Zwei Horizontalschnitte durch das Leitfähigkeitsmodell in der oberen bis mittleren Kruste.

\section{Mittlere bis untere Kruste}

Im Modell erstrecken sich die, die mittlere bis untere Kruste repräsentierenden Leitfähigkeiten zwischen 14 und $21 \mathrm{~km}$ Tiefe. Die modellierte Leitfähigkeitsverteilung in diesem Bereich ist in Abb. 3.5 dargestellt.

Im Modell wird die mittlere bis untere Kruste unter dem Array durch einen ausgedehnten 
Bereich anisotroper Leitfähigkeit dominiert. Dieser ist durch Lamellen ${ }^{4}$ modelliert, die in den Hintergrund von $40 \Omega$ m eingebettet sind. Dabei nimmt der spezifische Widerstand der Lamellen von $2 \Omega \mathrm{m}$ im Süden auf $10 \Omega \mathrm{m}$ im Norden zu. Die Richtung hoher Leitfähigkeit ist dabei $30^{\circ} \mathrm{N}$. Die Anpassung des guten Leiters in der mittleren Kruste und dessen Richtungsabhängigkeit erfolgt in guter Übereinstimmung mit Ergebnissen von JöDiCKE et al. (1983), OETTINGER (1994) und LEIBECKER et al. (2002).

Die im Modellschnitt dargestellte Struktur im nordöstlichen Teil des Arrays ist die sich bis in diese Tiefe fortsetzende, durch einen sehr gut leitenden Block modellierte, Göttinger D-Anomalie (PETER 1994). Insgesamt erstreckt sie sich im Modell im Tiefenbereich von $9.2-22 \mathrm{~km}$.

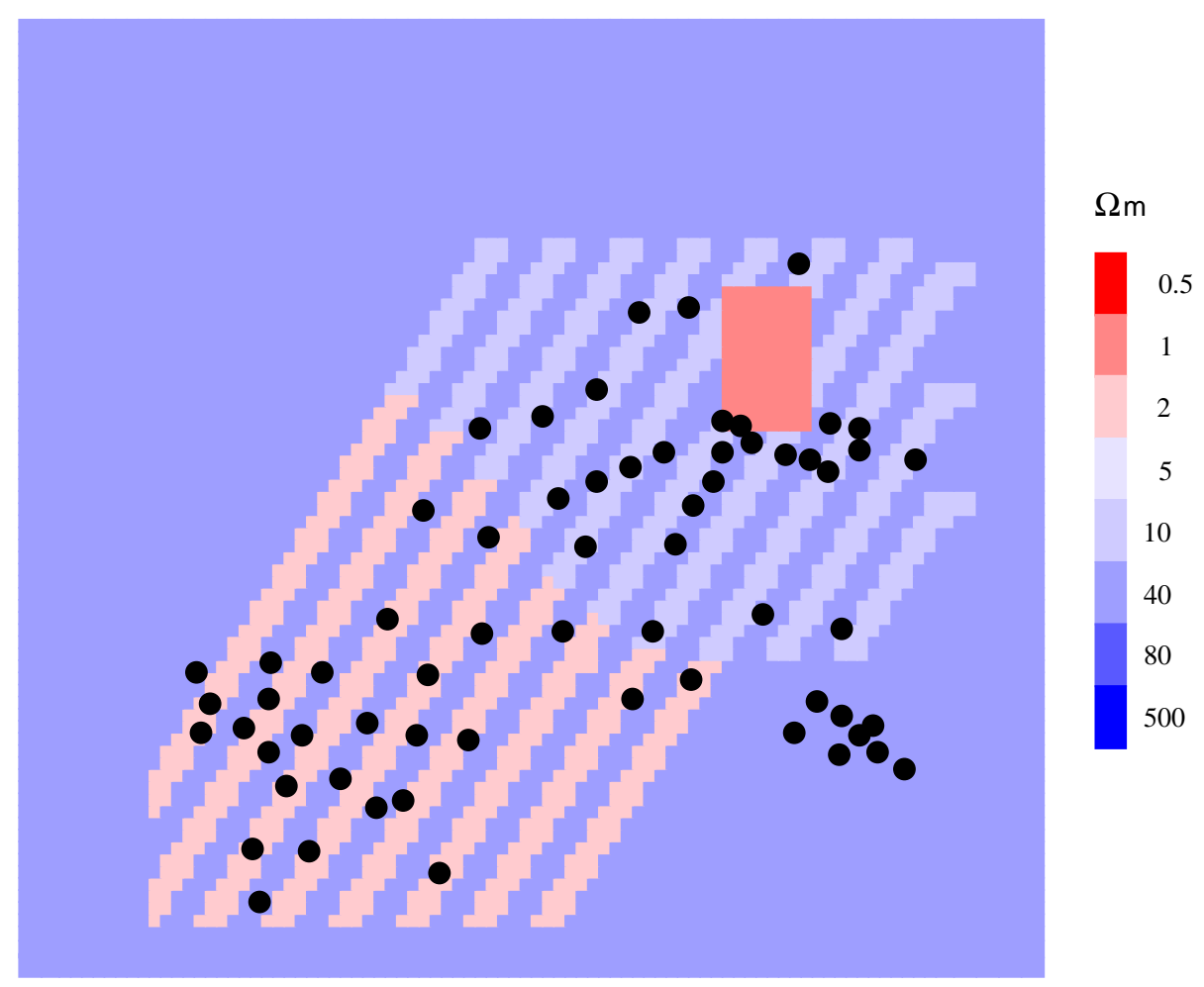

Abbildung 3.5: Horizontalschnitt der Modellschicht der mittleren bis unteren Kruste. Der anisotrope Bereich liegt in einer Tiefe von $14-21 \mathrm{~km}$. Die durch einen gutleitfähigen Block modellierte Göttinger D-Anomalie im nordöstlichen Teil erstreckt sich von 9.2 bis $22 \mathrm{~km}$.

\footnotetext{
${ }^{4}$ Zur Modellierung anisotroper Leitfähigkeiten durch Modellierungscodes, die nur isotrope Leitfähigkeiten zulassen, sei auf die Arbeiten von GROOM und BAILEY (1989b) und LEIBECKER (2000) verwiesen.
} 


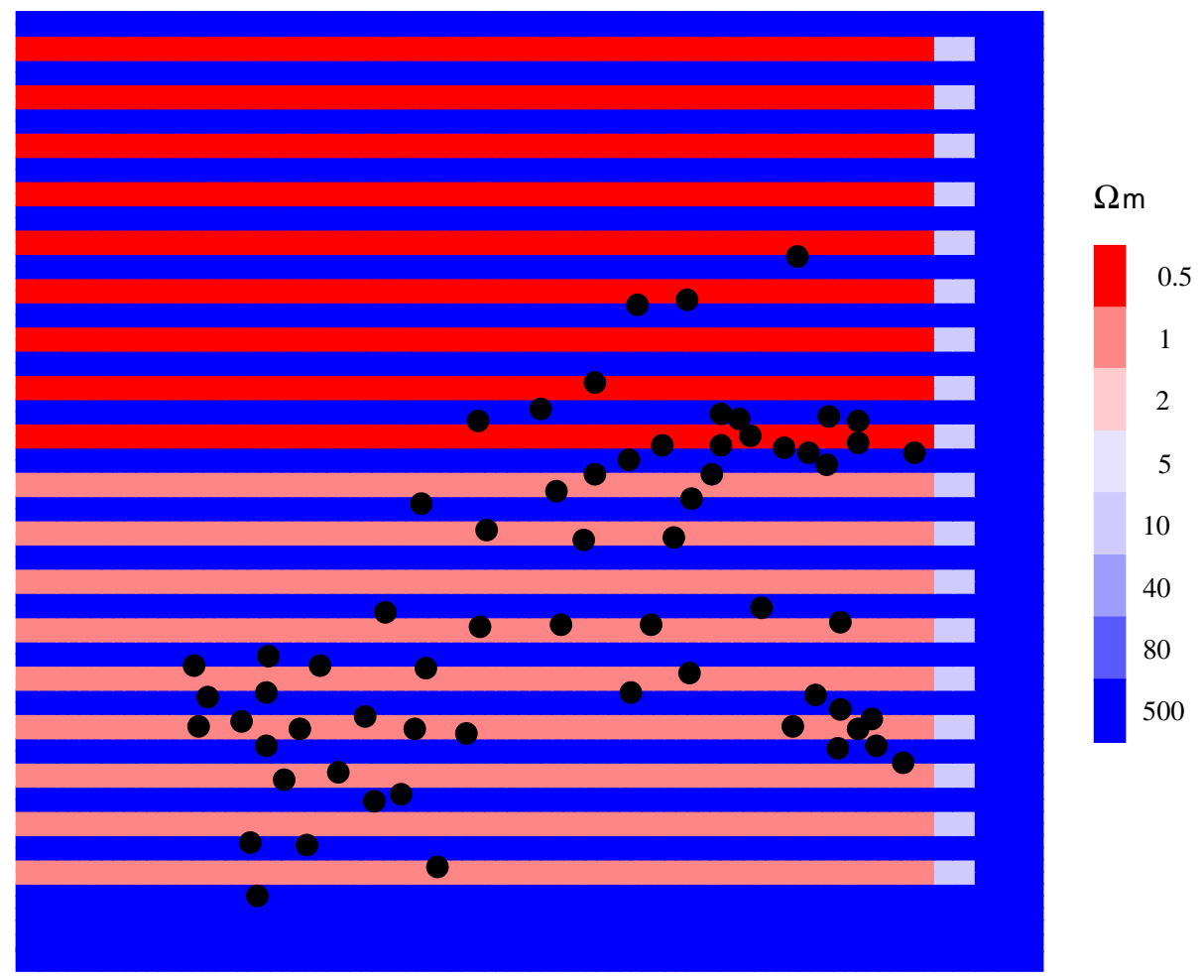

Abbildung 3.6: Horizontalschnitt durch die Modellschicht des oberen Mantels in einer Tiefe von $100-150 \mathrm{~km}$.

\section{Oberer Mantel}

Die in Kap. 2.3 in den Daten identifizierte starke Richtungsabhängigkeit der Leitfähigkeit bei langen Perioden ist durch eine anisotrope Schicht im Modell in einer Tiefe zwischen $100-150 \mathrm{~km}$ modelliert (Abb. 3.6). Dabei wird die Anisotropie durch Lamellen von $1 \Omega \mathrm{m}$ im Süden bzw $0.5 \Omega \mathrm{m}$ im Norden erzeugt, die in den gegenüber diesen Werten schlechtleitenden Hintergrund von $500 \Omega$ m eingebettet sind. Durch die Begrenzung der Phasenaufspaltung im östlichen Teil des Arrays wird auch im Modell die Ausdehnung der Lamellen nach Osten limitiert. Zur Vermeidung eines hohen Leitfähigkeitskontrastes am östlichen Rand der Lamellen erfolgt der Übergang durch Modellblöcke mittlerer Leitfähigkeit $(10 \Omega \mathrm{m})$. Im westlichen Teil ist durch die Begrenzung des Arrays keine Aussage über die Ausdehnung des Bereichs der Phasenaufspaltung möglich, daher wurden die Lamellen in westliche Richtung bis zum Modellrand fortgesetzt.

Das in diesem Kapitel vorgestellte Leitfähigkeitsmodell hat nicht den Anspruch, die im Bereich des Arrays vorhandenen Leitfähigkeiten abschließend zu erklären. Es ist vielmehr ein mögliches minimum structure model zur Anpassung der wichtigsten Eigenschaften der Daten und gibt prinzipiellen Aufschluss über die zugrundeliegenden Strukturen. Das eine genauere Modellierung und bessere Tiefeneingrenzung durch Modellstudien möglich ist, 
zeigt LEIBECKER (2000) durch detaillierte Parameterstudien von Tiefenlage und Leitfähigkeiten. Dabei ist im dreidimensionalen Modell die Unterscheidung zwischen Schichten unterschiedlicher integrierter Leitfähigkeit möglich. Im Rahmen des Fehlers können damit Aussagen über Tiefenlage und Leitfähigkeiten der interpretierten Strukturen getroffen werden.

\subsection{Modellergebnisse und Datenanpassung}

Die durch dreidimensionale Vorwärtsmodellierung an der Oberfläche des 3D Modells bestimmten Übertragungsfunktionen werden an dieser Stelle beschrieben. Um diese direkt mit den Messdaten vergleichen zu können, wurde entsprechend den Koordinaten des Modells (s. Abb. 3.2) eine Zuordnung zwischen Gitterpunkt und Messstation vorgenommen. Dabei ist jeder Station der nächstgelegene Gitterpunkt zugeordnet.

\subsubsection{Die Phase der Impedanz}

In Abb. 3.7 wird die Anpassung der gemessenen, unrotierten Phasenkurven der xy- und yx-Polarisation durch das Leitfähigkeitsmodell dargestellt. Die in Kap. 2.3 beschriebenen zwei Frequenzbereiche, für kurze $(<500 \mathrm{~s})$ und lange Perioden $(>1000 \mathrm{~s})$ finden sich auch in der Modellanpassung wieder.

Für kurze Perioden kann die für die mittlere bis untere Kruste modellierte einfache Leitfähigkeitsstruktur (Abb. 3.5) die lateralen Variationen im kurzperiodischen Bereich nicht quantitativ erklären. Jedoch gibt die Modellantwort qualitativ das Verhalten der unrotierten Phasenkurven wieder. Besonders im nördlichen Teil des Messgebietes macht sich für kurze Perioden der Einfluss der Sedimente der Norddeutschen Tiefebene (Abb. 3.4) auf die Daten bemerkbar. Diese hohe oberflächennahe Leitfähigkeit liefert an der nahe gelegenen Station ARN für kurze Perioden Phasenwerte beider Polarisationen kleiner $45^{\circ}$. Dieser Einfluss oberflächennaher Strukturen nimmt jedoch für längere Perioden $(>100 \mathrm{~s})$ schnell ab.

In der Modellantwort wirkt sich für lange Perioden der Einfluss der anisotropen Schicht im oberen Mantel (Abb. 3.6) in einer Aufspaltung der Phasenkurven aus. Diese Phasenaufspaltung beginnt bei Perioden von 500 s mit einem Maximum bei ca. 2000 s. Dabei bleibt die Phase der xy-Polarisation unter $45^{\circ}$ und folgt, von kleineren Abweichungen abgesehen, den Daten. Für die yx-Polarisation steigt von Süden nach Norden die modellierte Phase an. Dieses auch in den Daten beobachtete Ansteigen der Phase wird durch die im oberen Modell-Mantel nach Norden zunehmende Leitfähigkeit der Lamellen erklärt. Lediglich das Maximum der nördlichen Stationen ist durch das Modell nicht anzupassen. Besonders die großen Phasenwerte der yx-Polarisation an der Station BRI und die starke laterale Änderung dieses Maximums (im Vergleich zu den umliegenden Stationen) lassen auf einen Effekt, dessen Ursprung nicht ausschließlich in der Tiefe liegt, schließen. 

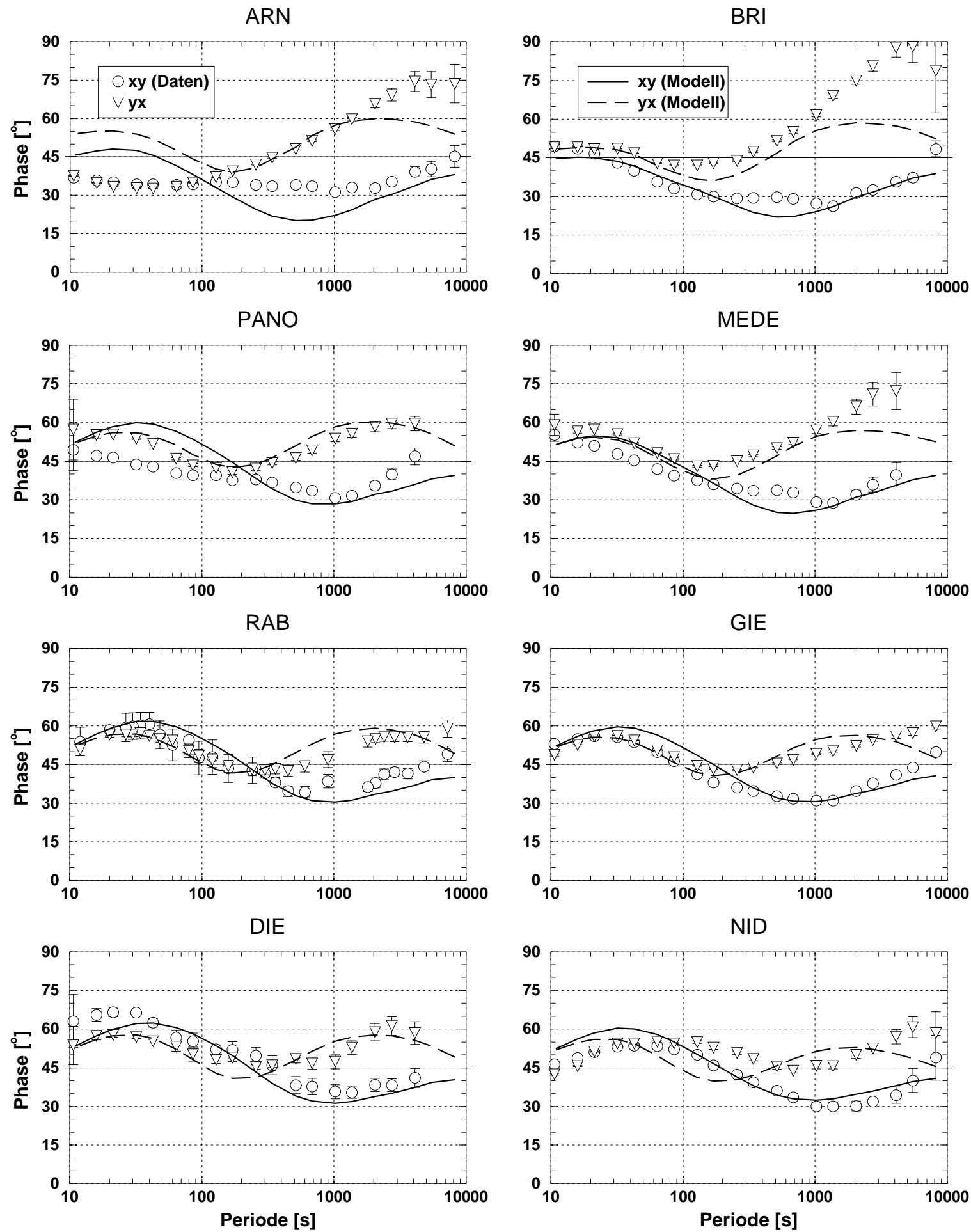

Abbildung 3.7: Unrotierte Phasenkurven der acht charakteristischen Stationen mit Modellantwort. 
Durch die flächenhafte Darstellung der langen Perioden (Abb. 3.8) wird deutlich, dass durch östliche Begrenzung der Lamellen die laterale Ausdehnung des Bereichs der Phasenaufspaltung in diese Richtung richtig wiedergegeben wird. Ebenfalls ist für lange Perioden durch die flächenhafte Darstellung der Einfluss oberflächennaher Strukturen sichtbar. Das ausgedehnte, gutleitfähige Körper an der Oberfläche auch für lange Perioden einen kleinen, jedoch nicht zu vernachlässigenden induktiven Anteil haben können, zeigen die Bereiche um die Göttinger D-Anomalie und die Krustenanomalie in der Eifel (vgl. Abb. 3.4). Die unterschiedlichen Geometrien dieser beiden Strukturen erklären deren unterschiedlich starken Einfluss auf die Polarisationen.

Dass das Leitfähigkeitsmodell trotz guter Anpassung die Daten nicht abschließend erklärt, zeigt der Vergleich von Abb. 3.8 mit 2.5. Nimmt in den Daten im Süden der Bereich der Phasenaufspaltung ab, wird dieses durch das Modell nicht angepasst. Dieses prinzipielle Problem der Erklärung von Effekten am Rand des Arrays zeigt sich gerade auch im nördlichen Bereich durch das Maximum der Phasenaufspaltung.
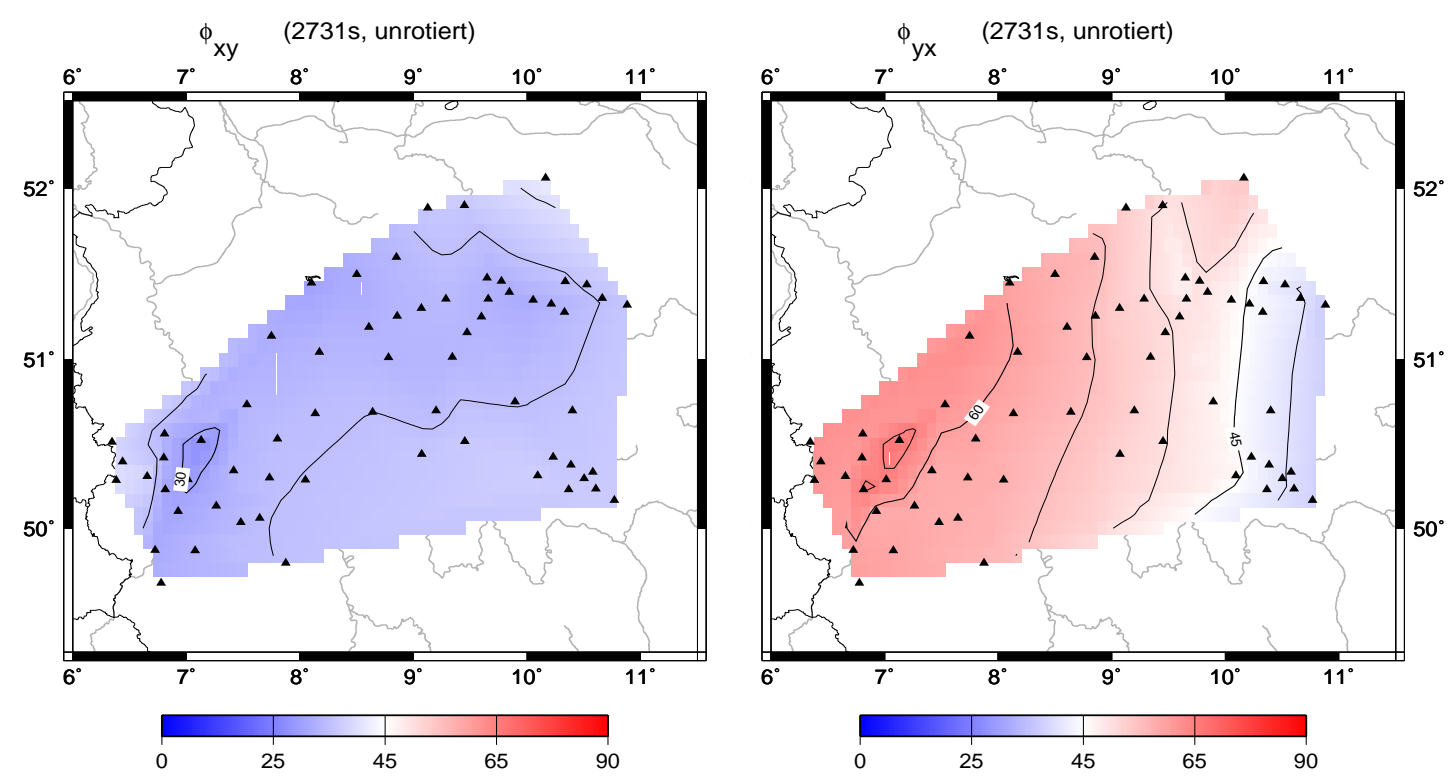

Abbildung 3.8: Modellantwort: Flächenhafte Darstellung der unrotierten Phasen der xyund yx-Polarisation bei der Periode von 2731 s.

\subsubsection{Strukturanpassung}

In Abb. 3.9 ist die Anpassung der phasensensitiven Winkel durch das Modell anhand der acht Stationen dargestellt. Inwieweit die anisotropen Schichten im Modell die als abschnittsweise zweidimensional interpretierten Daten erklären, zeigt sich neben der Anpassung der Phasen insbesondere auch am Frequenzgang des Rotationswinkels. 

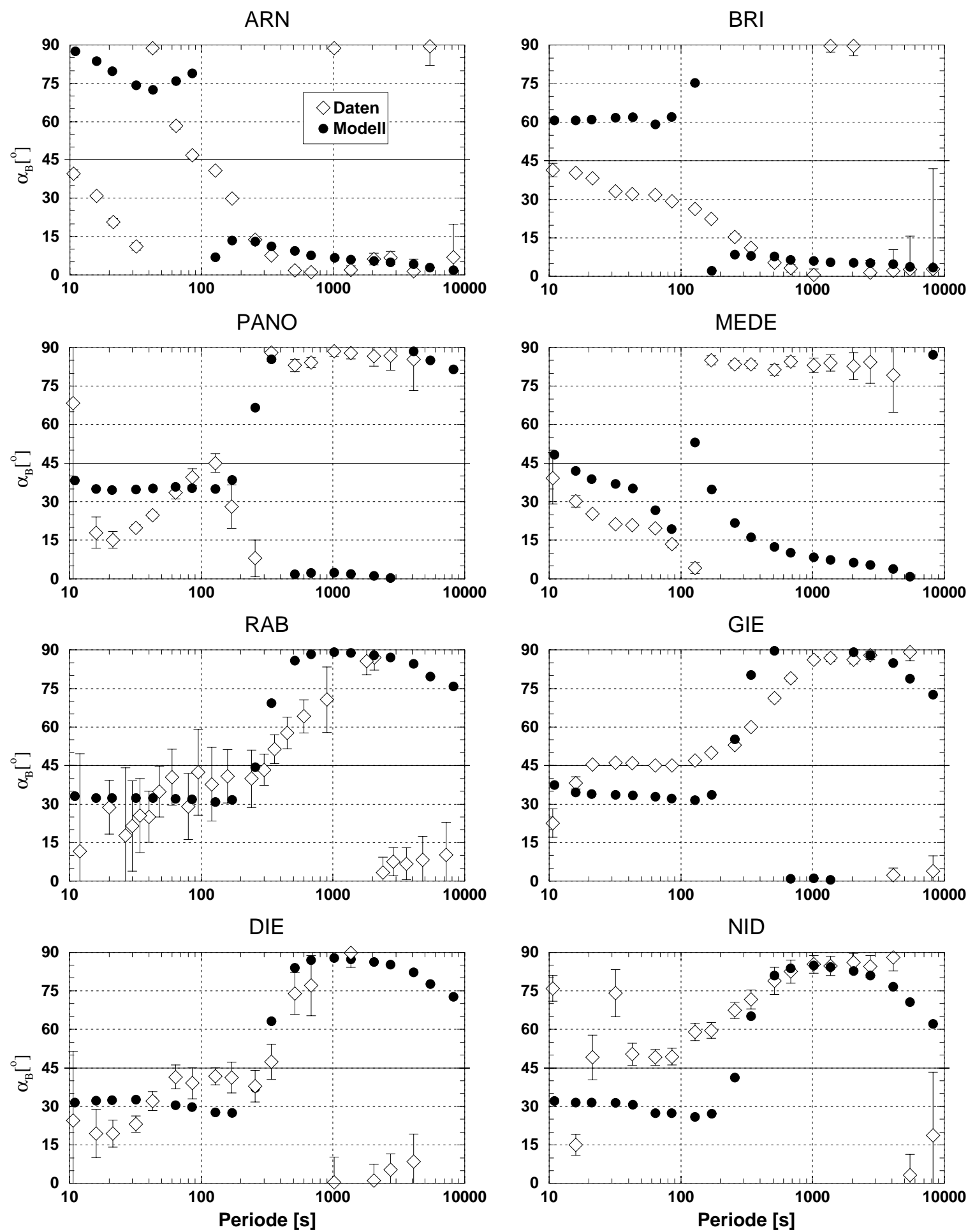

Abbildung 3.9: Phasensensitiver Rotationswinkel mit Modellantwort. Die offenen Quadrate stellen die Messdaten, die schwarzen Kreise die Modellantwort dar. 
Für kurze Perioden wird erneut deutlich, dass das für die Kruste sehr einfache Leitfähigkeitsmodell die komplizierten Strukturen im Detail nicht anpassen kann. Laterale krustale Variationen der Leitfähigkeit äußern sich in unterschiedlichen Frequenzgängen des Rotationswinkels benachbarter Stationen oder, wie z.B. bei der Station ARN durch starke Variation im Frequenzgang an einer Station selbst.

Bei langen Perioden nimmt für die Modellantwort erwartungsgemäß die Variation der Winkel ab. Die für den oberen Mantel modellierte WO-streichende Struktur passt durch die Rotationswinkel von $0^{\circ}$ bzw. $90^{\circ}$ die Daten an.

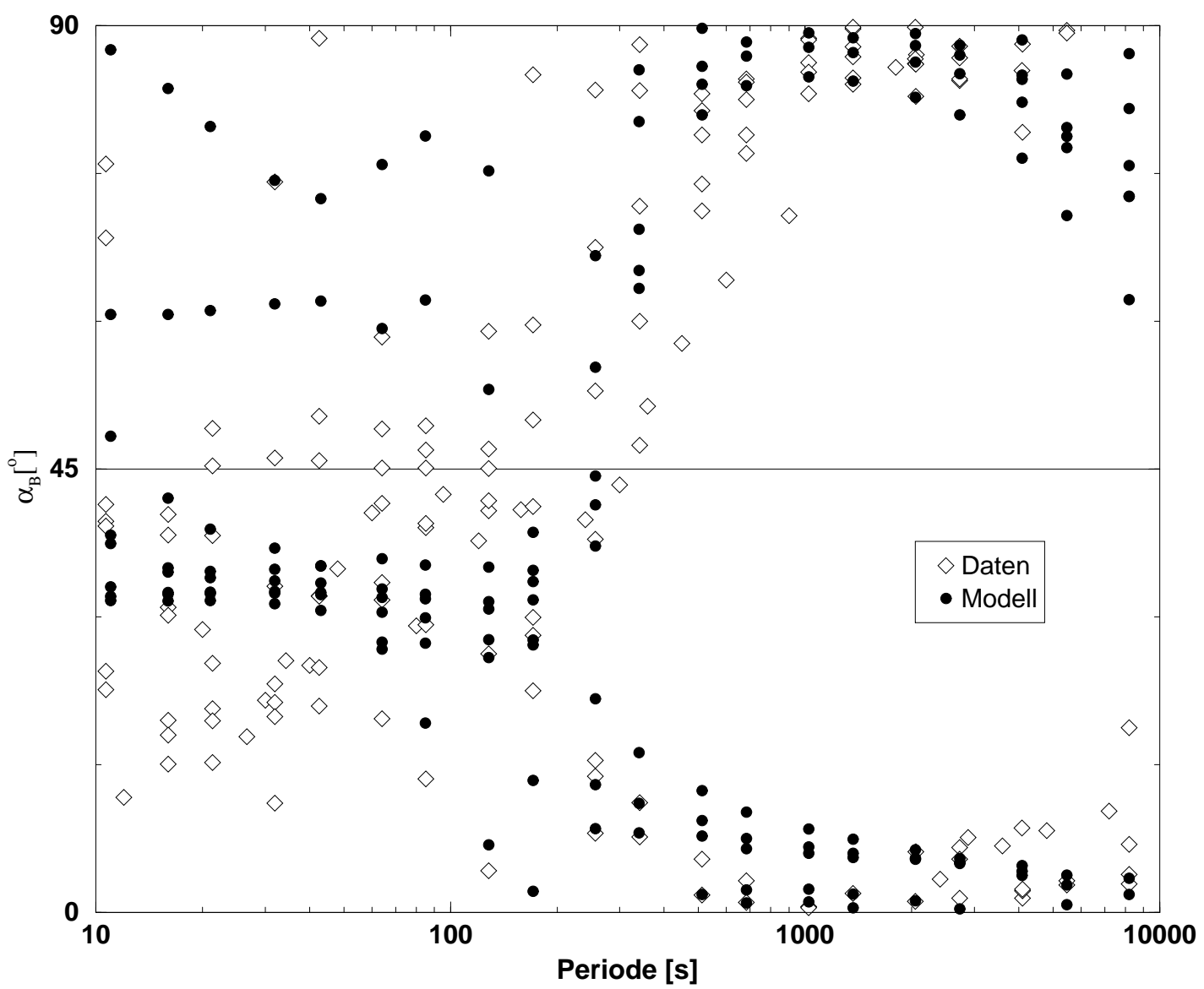

Abbildung 3.10: Gemeinsame Darstellung der phasensensitiven Rotationswinkel von Daten und Modellantwort der acht charakteristischen Stationen.

Hervorgehoben wird dies durch die gemeinsame Darstellung von Daten und Modellantwort der acht Stationen (Abb. 3.10). Die offenen quadratischen Symbole sind die Messdaten, die schwarzen Kreise die Modellantwort. In dem gemeinsamen Vergleich wird deutlich, dass das Modell die in der Diskussion der Daten gewonnenen Charakteristika im Frequenzgang erklärt. Die für kurze Perioden in den Daten zu beobachtenden Winkel zwischen $20^{\circ}$ und $45^{\circ}$ werden durch die Schicht der anisotropen Kruste mit einer 
$30^{\circ}$-Streichrichtung angepasst. Bei langen Perioden ist in der Modellantwort wie auch in den Daten ein über alle Stationen stabiler Winkel mit kleiner Streuung um $0^{\circ} / 90^{\circ} \mathrm{zu}$ beobachten. Dass die Tiefenlage beider anisotroper Schichten zu den Daten passt, zeigt sich im Bereich mittlerer Perioden. Dieser Übergangsbereich, in welchem die Perioden bei mittleren Eindringtiefen induktiv von beiden Schichten beeinflusst werden, stellt sich durch ändernde Rotationswinkel dar.

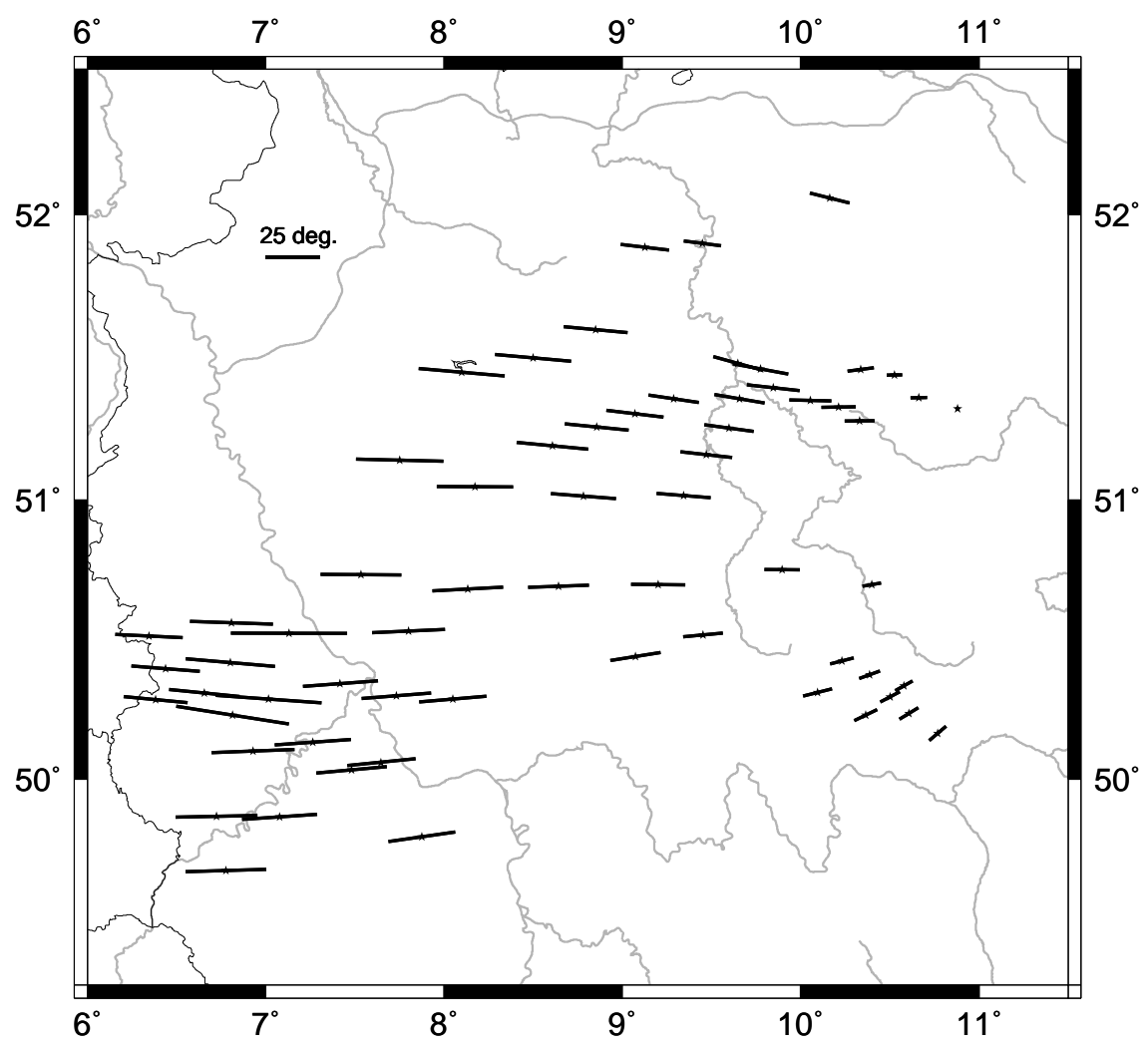

Abbildung 3.11: Differenz der rotierten Phasen der Modellantwort der xy- und yxPolarisation. Jede Station ist um den phasensensitiven Winkel rotiert; die Länge des Referenzbalkens ist $25^{\circ}$.

Auch bei der Modellantwort gilt den langen Perioden besonderes Interesse. In Abb. 3.11 sind Rotationswinkel und Phasendifferenzen für die langen Perioden anhand $2731 \mathrm{~s}$ flächenhaft dargestellt. Die Phasendifferenz ist (vgl. Abb. 2.9) im Koordinatensystem, das um den phasensensitiven Winkel gedreht ist, berechnet. Im westlichen Teil des Arrays zeigen alle Stationen einen sich lateral nur gering ändernden Drehwinkel. Beeinflusst durch die anisotrope Schicht im oberen Mantel weisen die Balken vorwiegend in WO-Richtung. Wie auch in den Daten, ist im östlichen Teil des Arrays ein in nördliche Richtung drehender Winkel zu beobachten. Dieser vorwiegend durch die Begrenzung 
der Lamellen verursachte Effekt ist in den Daten jedoch stärker ausgeprägt. Dies lässt auf eine kompliziertere östliche Begrenzung der anisotropen Struktur schließen, als im Modell dargestellt. Im Rheinischen Schiefergebirge zeigen sich in der Modellantwort kleinere laterale Variationen des Drehwinkels, die durch die krustale Struktur beeinflusst sind. Diese Variationen des Drehwinkels sind in den Daten jedoch ausgeprägter, was auch im Bereich der Kruste auf kompliziertere als im Modell angepasste Strukturen hinweist. Flächenhaft zeigt sich für die Phase des Modells, dargestellt durch die Länge der Balken, eine nach Osten abnehmende Aufspaltung beider Polarisationen, wie auch in den Daten (Abb. 2.9) beobachtet. Für die nördlichen Stationen ist diese, durch den möglichen Einfluß oberflächennaher Strukturen, in den Daten größer als im Modell. Im mittleren und südlichen Teil des Arrays haben die Aufspaltung in Modell und Daten gute Übereinstimmung. Im Westen ist sie im Modell größer als in den Daten beobachtet. Ursache hierfür sind die im Modell nach Westen bis zum Modellrand ausgedehnten gutleitfähigen Lamellen. Eine Erweiterung des Arrays nach Westen und eine damit verbundene mögliche Eingrenzung des Bereichs langperiodischer Phasenaufspaltung liessen eine genauere Anpassung auch im westlichen Teil zu.
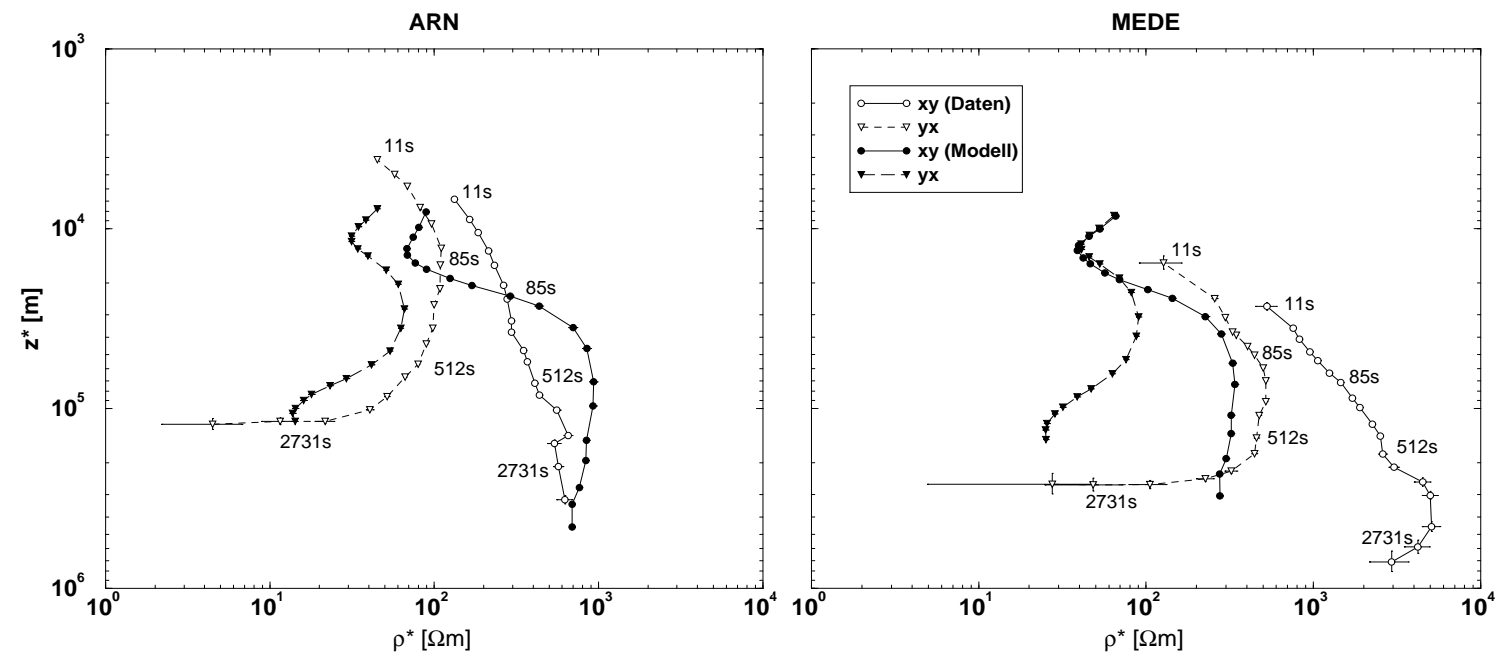

Abbildung 3.12: $\rho^{*}-z^{*}$-Darstellung der unrotierten Modellantwort der Stationen ARN und MEDE.

Abb. 3.12 zeigt für die Stationen ARN und MEDE im Vergleich die $\rho^{*}$-z*-Darstellung von Daten und Modellanpassung. Die in dieser Darstellung der Daten verdeutlichten Einflüsse von Verzerrung können durch das Modell nicht erklärt werden. Der Vergleich zeigt jedoch qualitativ, dass die Richtungsabhängigkeit der Leitfähigkeit durch das Modell richtig beschrieben wird. Für die yx-Polarisation werden auch in der Modellantwort die langen Perioden von einem guten Leiter beeinflusst und die größeren Eindringtiefen der xyPolarisation spiegeln den Einfluss hochohmiger Widerstände im Vergleich zu yx wider. 
Der Einfluss lateraler krustaler Leitfähigkeitsänderungen im Modell zeigt sich beim Vergleich der Modellantwort beider Stationen. Die oberflächennahen guten Leiter nördlich der Station ARN (vgl. Abb. 3.4) bewirken eine starke Verzerrung der $\mathrm{Z}_{x y}$-Komponente des Impedanztensors. Diese verursachen eine Verschiebung der zugehörigen $\rho^{*}-z^{*}$-Kurve hin zu höheren spezifischen Widerständen und größerem $z^{*}$. Auch die Modellantwort zeigt hier, dass eine quantitative Interpretation der Amplituden im Allgemeinen nicht sinnvoll ist.

\subsection{Einfluss krustaler Strukturen}

Um den Einfluss krustaler Strukturen, insbesondere der gutleitenden Sedimente der Norddeutschen Tiefebene auf die langperiodische Phasenaufspaltung zu beurteilen, sind in einem weiteren Modell die Leitfähigkeitsstrukturen der oberen bis mittleren Kruste (Abb. 3.4) entfernt worden. Ansonsten wurde das 3D-Leitfähigkeitsmodell unverändert über-
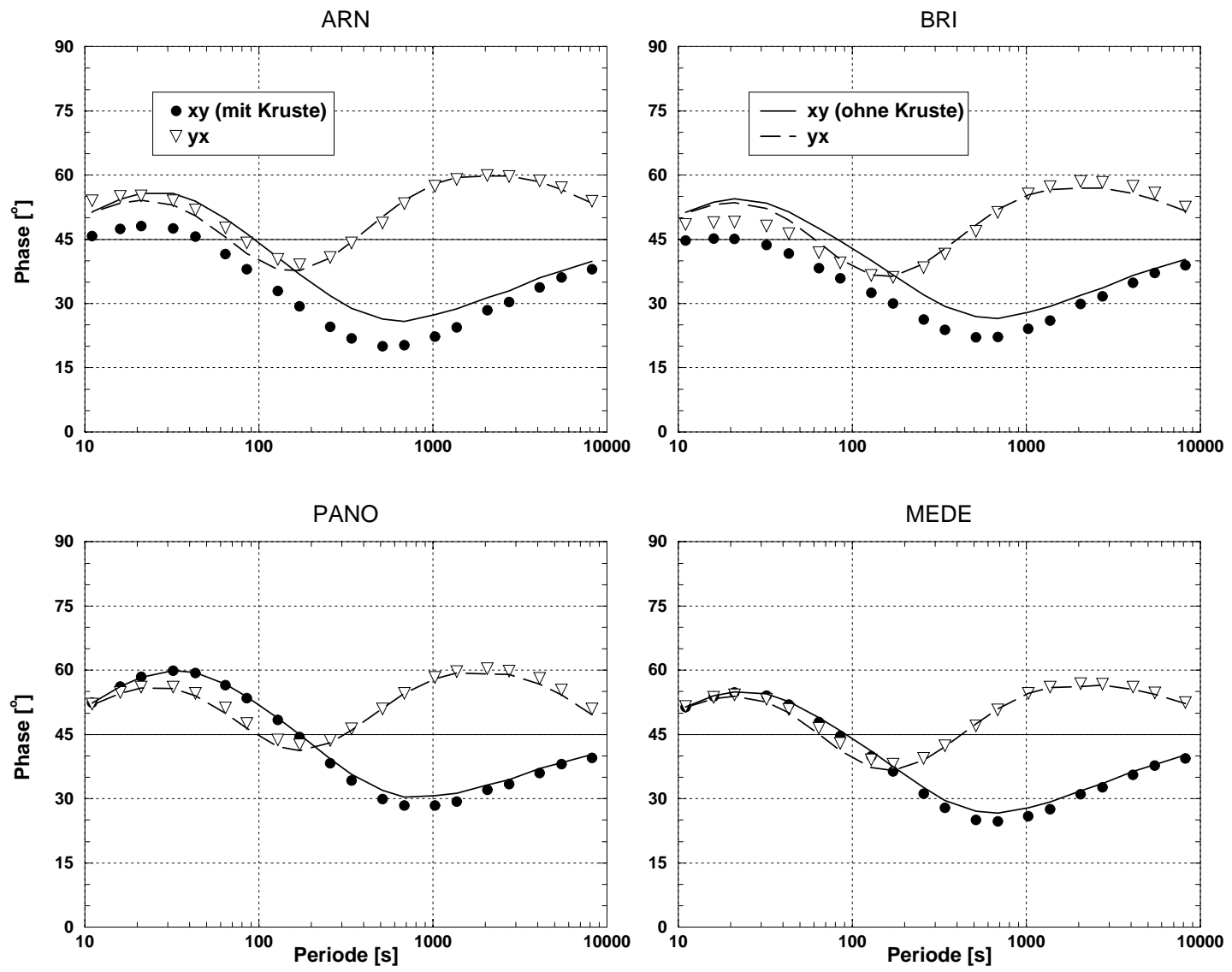

Abbildung 3.13: Einfluss der Leitfähigkeitsstrukturen der oberen bis mittleren Kruste anhand der unrotierten Phasenkurven der Stationen ARN, BRI, PANO und MEDE. 
nommen. Abb. 3.13 zeigt im Vergleich die Modellantwort der unrotierten Phasen beider Leitfähigkeitsmodelle für vier nördliche Stationen. Der Einfluss der oberen Kruste und dabei besonders der Sedimente der Norddeutschen Tiefebene (aufgrund der hohen integrierten Leitfähigkeit von bis zu 2200 S), nimmt nach Süden schnell ab. Ist dieser bei den Stationen ARN und BRI noch deutlich zu sehen, kann er weiter südlich bei PANO und MEDE schon vernachlässigt werden. Den größten Einfluss haben die oberflächennahen Strukturen erwartungsgemäß auf die kurzen Perioden. Der Vergleich beider Modelle zeigt, dass aufgrund des großen Induktionsraumes diese oberflächennahen Leitfähigkeitsänderungen auch einen Einfluss auf die langen Perioden haben. Dieser ist jedoch nicht groß genug, dass allein oberflächennahe Strukturen zur Erklärung des Bereichs großer Phasenaufspaltung ausreichten. Ein ähnlicher Einfluss zeigt sich auch beim Rotationswinkel, ist dort für lange Perioden aber auch zu vernachlässigen.

\subsection{Zusammenfassung: Ein anisotroper oberer Mantel?}

Die aus den dreidimensionalen Modellierungen gewonnenen Informationen werden hier zur weiteren Interpretation zusammengefasst.

- Die für die obere bis mittlere Kruste modellierten Strukturen beeinflussen nicht grundlegend die Aussagen über tiefliegende Leitfähigkeiten, sind jedoch zur sinnvollen Anpassung der Daten besonders für kurze Perioden wichtig.

- Für die mittlere bis untere Kruste gelingt die Anpassung der Daten durch eine einfache, gemittelte Struktur qualitativ. Der im Modell verwandte mittlere Winkel von $30^{\circ}$ sowie die Leitfähigkeiten stimmen mit anderen elektromagnetischen und geologischen Erkenntnissen überein (JÖDICKE et al. 1983; OETTINGER 1994; LEIBECKER et al. 2002). Da diese Arbeit nicht die Kruste zum Gegenstand hat, wird auf die Diskussion der Ursachen dieser hohen Leitfähigkeit auf die Literatur verwiesen (GlOVER und VINE 1994; YARDLEY und VALLEY 1997; SIMPSON 1999; SIMPSON 2001a).

- Für den oberen Mantel ergeben die Modellierungen einen weit ausgedehnten Bereich stark richtungsabhängiger Leitfähigkeit in einer Tiefe von $100-150 \mathrm{~km}$. Mit den aus den spezifischen Widerständen der Lamellen $\left(\rho_{1}, \rho_{2}\right)$ aus

$$
\rho_{\|}=\frac{2 \rho_{1} \rho_{2}}{\rho_{1}+\rho_{2}} \quad \text { und } \quad \rho_{\perp}=\frac{\rho_{1}+\rho_{2}}{2}
$$

berechneten Hauptwiderständen (oder den daraus berechneten inversen Hauptleitfähigkeiten) (EISEL und HAAK 1999) in Richtung und senkrecht zu den Lamellen 
von $\rho_{\|}=1-2 \Omega \mathrm{m}$ und $\rho_{\perp}=250 \Omega \mathrm{m}$ ergibt sich ein Anisotropiekoeffizient ${ }^{5}$ von $A>10$. Eine eindeutige Begrenzung des Bereichs der Aufspaltung ist nur nach Osten möglich. Um die Struktur dieser Leitfähigkeitsanomalie, besonders deren Mächtigkeit und Leitfähigkeit nicht zu überschätzen, sind die Lamellen im Westen unbegrenzt. Bei Erweiterung des Arrays und Eingrenzung des Bereichs der Phasenaufspaltung wird sich die ,integrierte Leitfähigkeit“ der Struktur parallel zu den Lamellen noch erhöhen.

- Der im Modell angepasste Tiefenbereich von 100 - $150 \mathrm{~km}$ und die dabei verwandten Leitfähigkeiten bilden ein mögliches Modell zur Erklärung der Daten. Die Oberkante der Mantelstruktur kann (zur Anpassung der Charakteristika in den Daten) zwischen 80 und $120 \mathrm{~km}$ variieren, wobei ihre Mächtigkeit in Abhängigkeit von den verwendeten Leitfähigkeiten bis zu $80 \mathrm{~km}$ betragen kann. Auf eine genaue Parameterstudie zur Eingrenzung der Modellparameter wird in dieser Arbeit verzichtet. Wie bei LEIBECKER et al. (2002) gezeigt, ist hierdurch eine genauere Bestimmung von Tiefenlage und Leitfähigkeiten des anisotropen Bereichs möglich. Die Gemeinsamkeit aller, die Daten erklärender Modelle ist die Lokalisierung des Bereichs stark anisotroper Leitfähigkeit auf den asthenosphärischen Teil des oberen Mantel mit einem Anisotropiekoeffizienten von $\approx 100$.

Bleibt abschließend noch die Frage zu klären, ob nur Anisotropie der Leitfähigkeit als mögliche Ursache die langperiodischen Daten erklären kann. Die Methode der Magnetotellurik allein kann, wie in Kapitel 1.4 beschrieben, in Abhängigkeit der Eindringtiefe nicht auf beliebig kleinen Skalen Leitfähigkeitsstrukturen auflösen (Diffusionsgleichung). Mit einem mehrere hundert Kilometer ausgedehnten Array ist jedoch die Unterscheidung zwischen 2D-Struktur oder Anisotropie im Mantel möglich. Der Versuch, durch kompliziertere Modelle mit zweidimensionalen Leitfähigkeitsstrukturen im Mantel sowohl die nach Norden zunehmende Phasenaufspaltung als auch die Induktionspfeile zu erklären, scheitert an der lateral konstanten Phase der xy-Polarisation (Kap. 2.3.1) bei langen Perioden. Die zur Erhöhung der yx-Phase führende hohe Leitfähigkeit in WO-Richtung wird von der xy-Phase im gesamten Array nicht registriert. Da diese für das gesamte Messgebiet bei langen Perioden nahezu keine Differenzen benachbarter Stationen zeigt, ist dies gleichbedeutend mit einer horizontal in NS-Richtung konstanten Leitfähigkeit im Bereich des Induktionsraumes.

Im Folgenden werden verschiedene Leitfähigkeitsmechanismen diskutiert, die als mögliche Ursachen der hohen Anisotropie und Leitfähigkeit in Betracht gezogen werden können.

\footnotetext{
${ }^{5}$ Der Anisotropiekoeffizient berechnet sich aus dem Verhältnis der beiden Hauptwiderstände $\mathrm{zu} A=$ $\sqrt{\rho_{\perp} / \rho_{\|}}($EISEL und HAAK 1999).
} 


\section{Leitfähigkeit und Anisotropie}

Zur Interpretation des im letzten Kapitel erhaltenen Leitfähigkeitsmodells bedarf es der Kenntnis der elektrischen Leitfähigkeit der Gesteine und Gesteinszusammensetzungen in Abhängigkeit der jeweiligen Druck- und Temperaturbedingungen.

Die meisten mineralogischen Modelle sehen Olivin als Hauptbestandteil (50 - 60 Vol. \%) des oberen Mantels (RINGWOOD 1975; IRIFUNE und RINGWOOD 1987; ITO und TAKAHASHI 1987). Es wird daher allgemein angenommen, dass Olivin als dominierend vernetzte Phase die Leitfähigkeit maßgeblich bestimmt (Duba und Constable 1993; Xu et al. 2000a). Für den oberen Mantel unter dem Meßgebiet kann davon ausgegangen werden, dass die mineralogische Zusammensetzung der eines Spinell-Lherzolith (Peridotitvarietät ${ }^{1}$ ), mit $75 \%$ Olivin $(\mathrm{Ol}), 17 \%$ Orthopyroxen (Opx), 7\% Klinopyroxen (Cpx) und 1\% Spinell entspricht (G. Witt-Eickschen, pers. Mitt.).

Bei der Betrachtung der Leitfähigkeitsmechanismen im oberen Mantel liegt das Hauptaugenmerk somit auf Olivin.

\subsection{Grundlagen}

In diesem Kapitel werden die Mechanismen diskutiert, die zur Beschreibung der Leitfähigkeiten von Gesteinen, Mineralien oder Schmelzen dienen. In dem in dieser Arbeit betrachteten Frequenzbereich $\left(\omega \ll 10^{3} \mathrm{~Hz}\right)$ gilt die quasistationäre Näherung und Verschiebungsströme können vernachlässigt werden (vgl. Kap. 1). Nur für höhere Frequenzen ist der Einfluss von Polarisationsphänomenen auf die Leitfähigkeit zu berücksichtigen (Guéguen und Palciauskas 1994).

\subsubsection{Leitfähigkeitsmechanismen}

Im Gegensatz zu einzelnen Atomen oder Molekülen sind in Festkörpern die Energieniveaus der Elektronen nicht diskret. Sie sind in Energiebändern angeordnet, getrennt

\footnotetext{
${ }^{1}$ Olivin-Orthopyroxen-Klinopyroxen Zusammensetzung mit einem Olivinanteil $>40 \%$, Hauptbestandteil des oberen Mantels.
} 


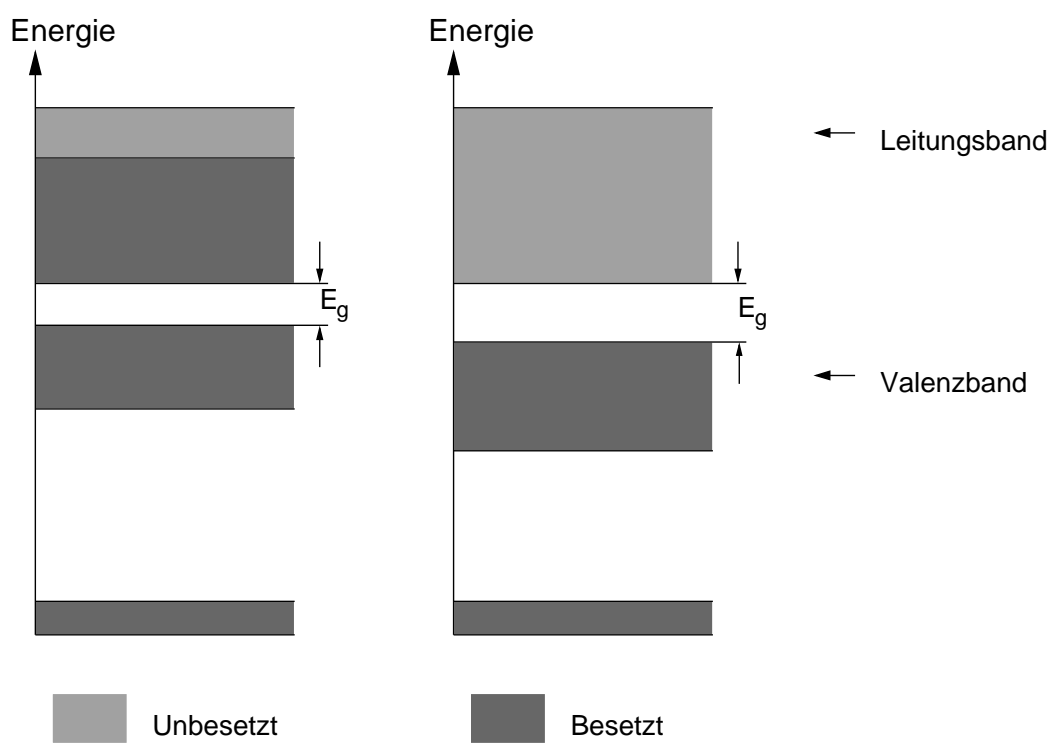

Abbildung 4.1: Schematische Darstellung der Energiezustände der Elektronen im Festkörper. Links: Metallischer Leiter, das Leitungsband ist teilweise besetzt. Rechts: Das Valenzband ist vollbesetzt, im Grundzustand ist das Leitungsband unbesetzt (Halbleiter: $\mathrm{E}_{g}<2.5 \mathrm{eV}$; Isolator: $\mathrm{E}_{g}>2.5 \mathrm{eV}$ ) (GuÉGuen und Palciauskas 1994).

durch Energiebereiche, in denen keine Elektronenzustände erlaubt sind (Bandlücken). Entsprechend der Besetzung dieser Bänder reagieren die Elektronen auf ein äußeres elektrisches Feld. Ist ein Band nur teilweise besetzt, haben die Elektronen Zugang zu einem Kontinuum von Zuständen. Angeregt durch ein äußeres elektrisches Feld driften sie mit der mittleren Geschwindigkeit $\bar{v}$ durch den Kristall oder Festkörper. Sind die Bänder hingegen vollbesetzt, besteht zum nächsten freien Band eine Energielücke $\mathrm{E}_{g}$, die überwunden werden muss.

Entsprechend ihrer elektrischen Leitfähigkeit werden Festkörper in drei Gruppen unterteilt: Metalle $\left(10^{5}<\sigma<10^{8} \mathrm{~S} / \mathrm{m}\right)$, Halbleiter $\left(10^{-7}<\sigma<10^{5} \mathrm{~S} / \mathrm{m}\right)$ und Isolatoren $\left(\sigma<10^{-7} \mathrm{~S} / \mathrm{m}\right)$.

In metallischen Leitern liegt das höchste Energieniveau (Fermi-Energie) gerade so, dass die Zustände in diesem Band nicht vollständig besetzt sind (vgl. Abb. 4.1, links). Die Elektronen in diesem Leitungsband sind nicht an ein besonderes Atom gebunden und frei beweglich. Diese freie Beweglichkeit ist in Metallen der Grund ihrer hohen elektrischen Leitfähigkeit. Neben Metallen zeigen auch andere Materialen wie z.B. Graphit diese metallische Leitfähigkeit.

Bei Halbleitern und Isolatoren sind alle Bänder vollbesetzt (Abb. 4.1, rechts). Die Lücke zwischen der Oberkante des Valenz- und der Unterkante des Leitungsbandes wird Bandlücke genannt. Bei Halbleitern ist diese Bandlücke relativ klein $(\sim 1 \mathrm{eV})$. 
Durch thermische Anregung können Elektronen aus dem Valenz- in das Leitungsband angehoben werden. Sowohl die Elektronen im Leitungsband als auch die nun unbesetzten Zustände im Valenzband tragen zur Leitfähigkeit bei. Bei höheren Temperaturen gehören viele Silikate und Oxide zu den Halbleitern.

Bei Isolatoren beträgt diese Bandlücke oft mehr als $5 \mathrm{eV}$ und verhindert so die thermische Anregung der Elektronen in das Leitungsband. Hier beeinflussen dann meist Störstellen im Kristall (Fremdatome oder Kristallfehler) dessen Leitfähigkeit. Diese Störstellenleitung ist bei tiefen Temperaturen oft der dominierende Mechanismus, kann bei hohen Temperaturen jedoch durch thermisch angeregte ionische Leitfähigkeit des reinen Kristalls übertroffen werden. Die meisten der in der Erde vorkommenden Mineralien sind unter den dort vorherrschenden Temperatur- und Druckbedingungen Isolatoren.

Die elektrische Leitfähigkeit in Festkörpern resultiert allgemein aus der Bewegung von Ladungsträgern in Richtung des Gradienten eines elektrischen Potentials. Ein Diffusionsstrom in $\mathrm{x}$-Richtung wird beschrieben durch

$$
J=-D \frac{\partial n}{\partial x}+\bar{v} n
$$

mit dem Diffusionsanteil $D \frac{\partial n}{\partial x}$ (FICK'sches Gesetz) und dem Konvektionsanteil $\bar{v} n$. Dabei ist $\mathrm{D}$ die Diffusionskonstante, $\mathrm{n}$ die Verteilung der Teilchen und $\bar{v} n$ deren mittlere Geschwindigkeit.

Unter der Annahme eines stationären Systems ist der Gesamtfluss gleich null, $\mathrm{J}=0$. Im thermischen Gleichgewicht folgt die räumliche Verteilung der Teilchen $n$ einer Boltzmannverteilung

$$
n(x)=n_{0} \cdot e^{-\varphi / k_{B} T},
$$

mit dem elektrischen Potential $\varphi=q V$, der Temperatur $T$ und der Boltzmannkonstante $k_{B}$. Hieraus folgt

$$
\frac{d n}{d x}=-\frac{n}{k_{B} T} \frac{d \varphi}{d x}=\frac{n F}{k_{B} T}
$$

wobei $F=-\frac{d \varphi}{d x}$ die den konvektiven Transport antreibende Kraft ist. Aus (4.1), (4.2) und $J=0$ folgt

$$
\frac{\bar{v}}{D}=\frac{F}{k_{B} T}
$$

Für den stationären Fall erhält man für die Bewegung der Teilchen im elektrischen Feld E eine mittlere Driftgeschwindigkeit der Elektronen $\bar{v}=\mu E$. Die Mobilität der Ladungsträger $\mu$ ist dabei abhängig vom Teilchentyp und dem Einfluss des Mediums. Für die Stromdichte j gilt mit (4.3) und $F=q \cdot E$

$$
j=n q \bar{v}=n q^{2} \frac{D}{k_{B} T} E
$$


und aus (1.1) folgt die NERNST-EINSTEIN-Gleichung

$$
\sigma=n q^{2} \frac{D}{k_{B} T}
$$

Der Diffusionskoeffizient D folgt dabei einem Exponentialgesetz der Form

$$
D=D_{0} e^{-\Delta H / k_{B} T},
$$

mit der Aktivierungsenthalpie $\Delta H=\Delta U+p \Delta V$, der Aktivierungsenergie $\Delta U$, dem Druck $\mathrm{p}$ und dem Aktivierungsvolumen $\Delta V$. Für die Leitfähigkeit folgt daraus

$$
\sigma=\sigma_{0} e^{-\Delta H / k_{B} T},
$$

mit dem exponentiellen Vorfaktor $\sigma_{0}$.

\subsubsection{Effektive Leitfähigkeit von Gesteinen}

Die Leitfähigkeit von Gesteinen hängt in hohem Maße von deren Mikrostruktur ${ }^{2} a b$. Grund sind die z.T. um mehrere Größenordnungen variierenden Leitfähigkeiten der einzelnen gesteinsbildenden Minerale. Zur Berechnung der mittleren Leitfähigkeit eines Volumens reicht demnach allein die Kenntnis der mittleren Zusammensetzung und deren Leitfähigkeiten nicht aus, es muss zusätzlich auch deren Verteilung berïcksichtigt werden.

Im Inneren eines Gesteinsvolumens gilt lokal das Ohmsche Gesetz:

$$
\vec{j}(\vec{r})=\sigma(\vec{r}) \vec{E}(\vec{r})=-\sigma(\vec{r}) \vec{\nabla} V(\vec{r}) .
$$

Das Potential $V(\vec{r})$ ist dabei von der Leitfähigkeitsverteilung in dem Volumen abhängig. Für eine nach aussen makroskopisch homogene und isotrope Probe wird die effektive Leitfähigkeit $\sigma_{e f f}$ definiert. Zwischen zwei parallelen Ebenen im Abstand L wird bei anliegender Potentialdifferenz $\Delta V$ die mittlere Stromdichte $<\vec{j}>$ gemessen:

$$
<\vec{j}>\equiv \frac{1}{A} \iint_{S}(\vec{J} \cdot \hat{z}) d S \equiv \sigma_{\text {eff }}\left(\frac{\Delta V}{L}\right),
$$

A ist über die zu integrierende Fläche und $\hat{z}$ ein zu dieser Fläche senkrechter Einheitsvektor. Ist die mikroskopische Leitfähigkeitsverteilung $\sigma(\vec{r})$ bekannt, kann hieraus die effektive Leitfähigkeit direkt berechnet werden:

$$
\sigma_{e f f} \equiv\left(\frac{L}{\Delta V}\right) \frac{1}{A} \iint_{S} \sigma(\vec{r})(-\hat{z} \cdot \vec{\nabla} V) d S .
$$

\footnotetext{
${ }^{2}$ Das natürliche Vorkommen von Gesteinen ist eine Mischung verschiedener, normalerweise inhomogen verteilter Minerale. Ein reines Mineral, ohne Fehlstellen, wird ein Einkristall genannt. Ein Polykristall ist eine Anordnung anisotrop und zufällig ausgerichteter Einkristalle. Von polykristallinem Aggregat spricht man, wenn Einkristalle unterschiedlicher anisotroper Minerale zufällig vermischt sind (BERRYMAN 1995).
} 
Für statistische Verteilungen von $\sigma(\vec{r})$ ist eine direkte Berechnung nicht mehr möglich. Ausgehend von einfachen Geometrien in Zweiphasensystemen kann $\sigma_{e f f}$ näherungsweise berechnet oder durch obere und untere Grenzen eingeschränkt werden. Ein Beispiel für ein solches Mischungsgesetz geht auf HASHIN und SHTRIKMAN (1962) zurück. Dabei benutzten sie ein Medium, das aus Kugeln der Leitfähigkeit $\sigma_{1}$, überzogen mit einer dünnen Schale der Leitfähigkeit $\sigma_{2}$, zusammengesetzt war. Das Volumenverhältnis der beiden Phasen ist $c_{1}=\left(1-c_{2}\right)$ zu $c_{2}$. Um den gesamten Raum mit Kugeln zu füllen, geht im Grenzwert deren Radius gegen null und deren Anzahl gegen unendlich. Mit $\sigma_{1}>\sigma_{2}$ folgt für die untere $\left(\mathrm{HS}^{-}\right)$und obere $\left(\mathrm{HS}^{+}\right)$HASHIN-SHTRIKMAN-Grenze:

$$
\begin{aligned}
\sigma_{\mathrm{HS}^{-}} & =\sigma_{2}+c_{1}\left[\frac{1}{\sigma_{1}-\sigma_{2}}+\frac{c_{2}}{3 \sigma_{2}}\right]^{-1} \\
\sigma_{\mathrm{HS}^{+}} & =\sigma_{1}+c_{2}\left[\frac{1}{\sigma_{2}-\sigma_{1}}+\frac{c_{1}}{3 \sigma_{1}}\right]^{-1},
\end{aligned}
$$

mit $\sigma_{\mathrm{HS}^{-}}<\sigma_{e f f}<\sigma_{\mathrm{HS}^{+}}$.

Neben der Angabe einer oberen und unteren Grenze kann für spezielle Geometrien auch direkt die effektive Leitfähigkeit berechnet werden. Weitere Mischungsgesetze wichtiger Geometrien von Zwei- oder Mehrphasensystemen sind in Anh. A.3 zusammengestellt.

\subsection{Leitfähigkeiten im oberen Mantel}

Zur Beschreibung von Mantelleitfähigkeiten wird in Labormessungen an Gesteinsproben oder synthetischen Zusammensetzungen versucht, deren Temperatur- und Druckabhängigkeit aufzulösen. Dabei haben die mineralische Zusammensetzung, der Sauerstoffpartialdruck, Wassergehalt oder Anteil an Schmelze in der Probe einen entscheidenden Einfluss.

Aufgrund der komplexen Zusammensetzung des Erdmantels ist auch die Leitfähigkeit ein Zusammenspiel von vielen Leitungsmechanismen. Für unterschiedliche Gesteine zeigen Auftragungen von $\log (\sigma)$ gegen $\mathrm{T}^{-1}$ mehrere Bereiche linearen Verhaltens. Dies deutet den temperaturabhängigen Einfluss unterschiedlicher Ladungsträger als dominierenden Leitungsmechanismus an (TYBURCZY und FISLER 1995). Allgemein gilt:

$$
\sigma=\sum_{i} \sigma_{i}=\sum_{i} \sigma_{0}^{i} e^{-\Delta H_{i} / k_{B} T}
$$

Im Folgenden werden für die wichtigsten Regime im oberen Mantel anhand von theoretischen Überlegungen, Labor- und Feldmessungen Aussagen über deren Leitfähigkeiten gemacht. 


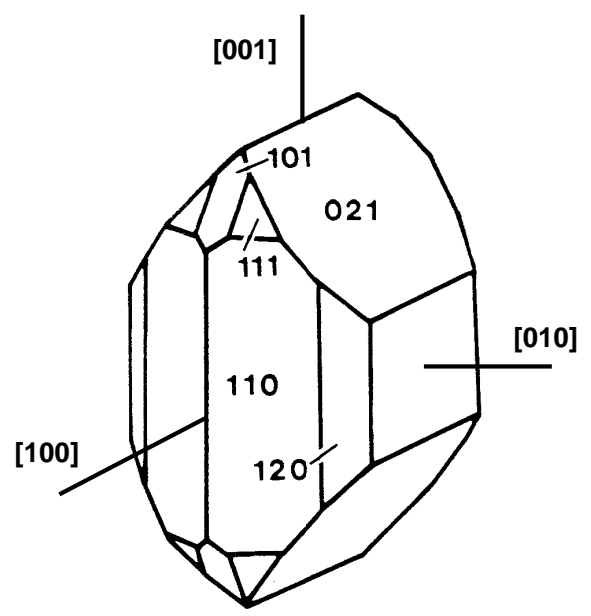

Abbildung 4.2: Die Hauptachsen des Olivinkristalls (nach MATTHES 1996)

\subsubsection{Trockener Olivin}

Die Temperatur- und Druckabhängigkeit der Leitfähigkeit von trockenem Olivin (ohne Fluide oder Schmelzen) ist seit langem Gegenstand geophysikalischer Untersuchungen (RUNCORN und TOZER 1955). In früheren Messungen war die Leitfähigkeit noch unter z.T. ungenau bekannten thermodynamischen Verhältnissen bestimmt (DubA 1972; DubA 1976; DubA 1982). In aktuellen Messungen werden auch die Abhängigkeit von Eisen $\mathrm{Fe} /(\mathrm{Mg}+\mathrm{Fe}$ ) (HIRSCH und SHANKLAND 1991a; HIRSCH und SHANKLAND 1991b), der die Leitfähigkeit stark beeinflussende Sauerstoffpartialdruck $f_{O_{2}}$ (DuBA et al. 1973; DuBA et al. 1974) oder der Einfluss des die Probe bei den Messungen umschließenden Puffers (WANAMAKER und DuBA 1993) mit berücksichtigt. Zur Beschreibung der Leitfähigkeiten von Gesteinen sind darüber hinaus die unterschiedlichen Ergebnisse der Messungen an ein- und polykristallinen Proben zu beachten (ROBERTS und TYBURCZY 1991; ROBERTS und TYBURCZY 1993; Duba und CONSTABLE 1993).

Weitgehende Übereinstimmungen der Labormessungen ermöglichten die Aufstellung einer Referenz-Kurve ${ }^{3} \mathrm{SO} 2$ für die Temperaturabhängigkeit der Leitfähigkeit isotropen Olivins (CONSTABLE et al. 1992). Diese wird als geometrisches Mittel (A.2) der von SHANKLAND und DUBA (1990) entlang der drei Hauptachsen ${ }^{4}$ (s. Abb. 4.2) im Olivinkristall bestimmten Leitfähigkeiten berechnet. Im Temperaturbereich $720^{\circ}-1500^{\circ} \mathrm{C}$ gilt

$$
\bar{\sigma}_{S O 2}=10^{2.402} e^{-1.60 e V / k_{B} T}+10^{9.17} e^{-4.25 e V / k_{B} T} .
$$

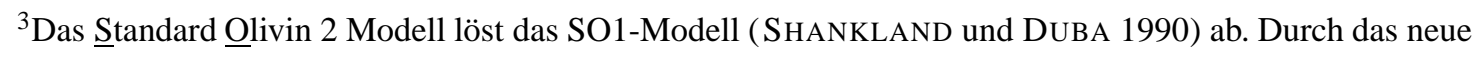
Modell werden auch die Daten bei tieferen Temperaturen $\left(<1000^{\circ} \mathrm{C}\right)$ angepasst.

${ }^{4}$ Dabei ist [ijk] die auf der Kristallfläche (ijk) senkrecht stehende Achse. Die Hauptachsen [100], [010] und [001] werden in der Literatur auch mit a-, b- und c-Achse bezeichnet.
} 


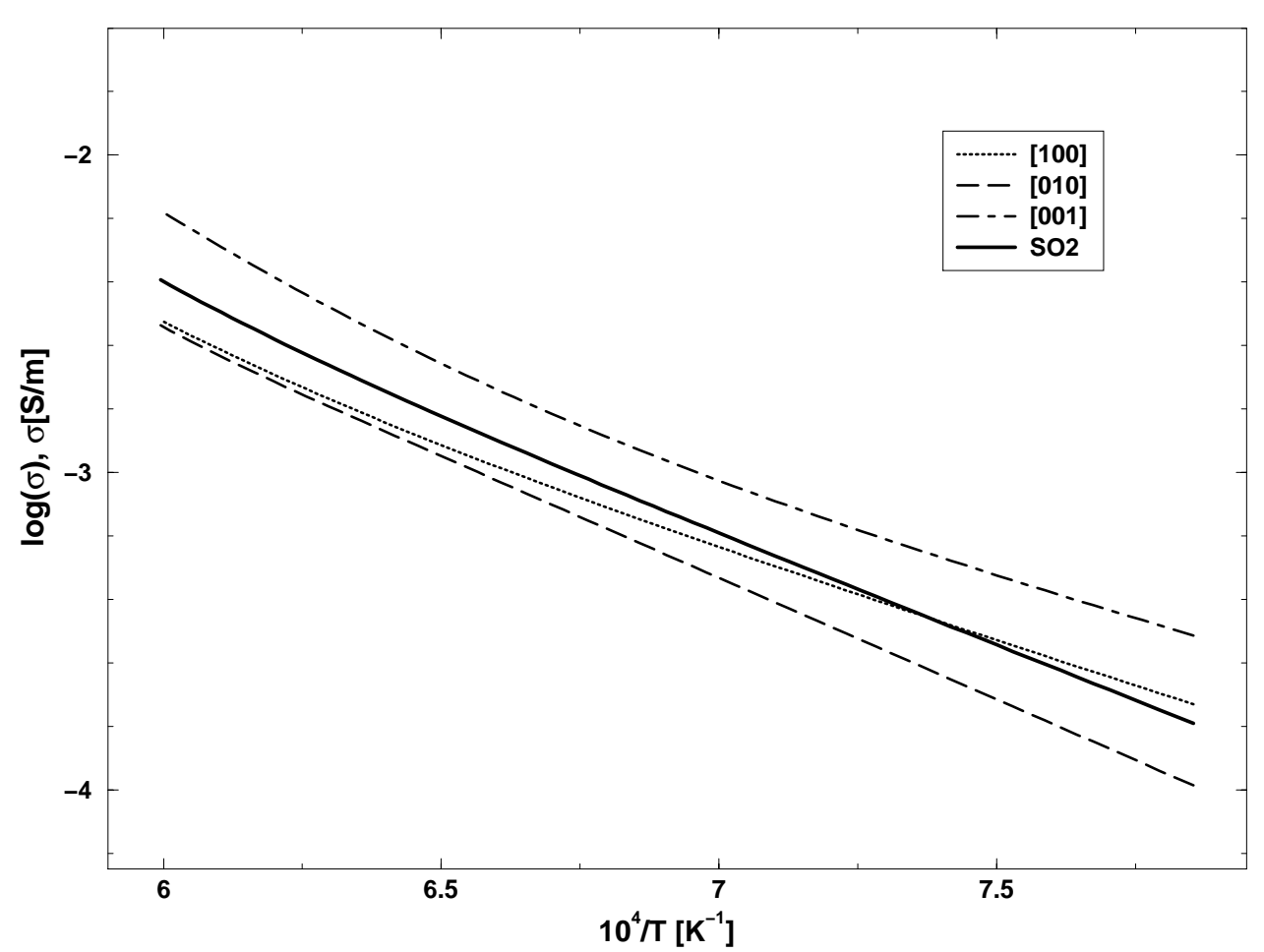

Abbildung 4.3: Aus Labormessungen bestimmte Leitfähigkeit von Olivin entlang der drei Hauptachsen (SHANKLAND und DUBA 1990) und die für isotropen Olivin berechnete SO2-Modellkurve (CONSTABLE et al. 1992).

Die Anpassung durch zwei Terme entspricht hier mindestens zwei sich überlagernden Leitfähigkeitsmechanismen (vgl. 4.5). In diesem Modell ist $\sigma$ druckunabhängig, vermutlich aufgrund sich gegenseitig aufhebender Druckabhängigkeiten der verschiedenen Mechanismen (SCHOCK et al. 1989).

In Abb. 4.3 sind die Leitfähigkeiten entlang der drei Hauptachsen und die des SO2Modells dargestellt. In trockenem Olivin dominiert bei tiefen Temperaturen die elektronische Leitung durch Übergang von $\mathrm{Fe}^{2+} \mathrm{zu} \mathrm{Fe}^{3+}$ unter Abgabe eines Elektrons an das Leitungsband (Kleines Polaron), bei hohen Temperaturen die ionische Leitung durch

\begin{tabular}{lcccc}
\hline Probe & $\begin{array}{c}P \\
(\mathrm{GPa})\end{array}$ & $\begin{array}{c}T \\
\left({ }^{\circ} \mathrm{C}\right)\end{array}$ & $\begin{array}{c}\sigma_{0} \\
(\mathrm{~S} / \mathrm{m})\end{array}$ & $\begin{array}{c}\Delta \mathrm{H} \\
(\mathrm{eV})\end{array}$ \\
\hline Olivin & $4-10$ & $1000-1400$ & 490 & $1.62 \pm 0.04$ \\
Orthopyroxen & 5 & $1000-1400$ & 5248 & $1.80 \pm 0.02$ \\
Klinopyroxen & 13 & $1000-1400$ & 1778 & $1.87 \pm 0.02$ \\
Ilmenit/Granat & 21 & $1200-1500$ & 2239 & $1.66 \pm 0.03$ \\
\hline
\end{tabular}

Tabelle 4.1: Aktivierungsenthalpien und Vorfaktoren gesteinsbildender Minerale (nach XU et al. 2000b). 


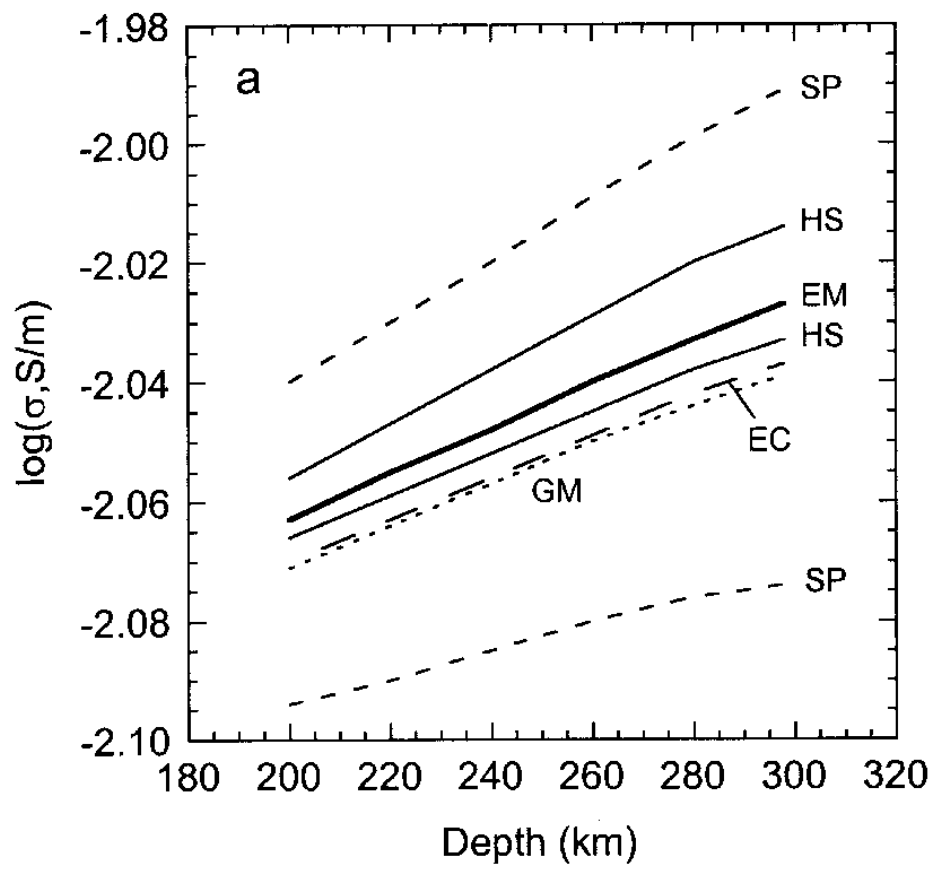

Abbildung 4.4: Effektive Leitfähigkeiten für den Tiefenbereich 200-300 km. Für die verwendete Mantelzusammensetzung in diesen Tiefen und den (in Hochdruckexperimenten bestimmten) Leitfähigkeiten einzelner Minerale sind für verschiedene Mischungsgesetze (s. Anh. A.3) die effektiven Leitfähigkeiten berechnet (XU et al. 2000b).

Mg-Lücken (SCHOCK et al. 1989). Dabei variieren die Leitfähigkeiten entlang der drei Achsen im Olivinkristall maximal um den Faktor $\sigma_{[001]} / \sigma_{[010]} \approx 2.3$. Die Richtung hoher Leitfähigkeit ist entlang der [001]-, die der geringsten Leitfähigkeit entlang der [010]Achse.

Die Messung der Leitfähigkeit in Abhängigkeit hoher Drucke und Temperaturen war lange aufgrund des fehlenden thermodynamischen Gleichgewichts in der Hochdruckzelle nicht möglich (XU et al. 2000a). In neueren Hochdruckmessungen kann, dank Verwendung anderer, die Probe umgebender Puffer, die Druckabhängigkeit der Leitfähigkeit unter Mantelbedingungen bestimmt werden (XU et al. 1998b; XU et al. 1998a; XU und SHANKLAND 1999; XU et al. 2000a).

Die für Olivin im Temperaturbereich von $1000^{\circ}-1400^{\circ} \mathrm{C}$ und Drucken von $4-10 \mathrm{GPa}$ gemessenen Paramter stimmen mit denen früherer Messungen (XU et al. 2000a) bei $1 \mathrm{~atm}$ überein. In Tab. 4.1 sind diese für Olivin und die wichtigsten Mantelminerale bei entsprechenden Temperaturen und Drucken dargestellt. Basierend auf den nun für die Mantelminerale vorliegenden Labormessungen konnten XU et al. (2000b) für verschiedene Tiefenbereiche und Zusammensetzungen aus unterschiedlichen Mischungsgesetzen 
(s. Anh. A.3) effektive Leitfähigkeiten berechnen. In Abb. 4.4 sind die Ergebnisse im Tiefenbereich $200-300 \mathrm{~km}\left(1380^{\circ}-1420^{\circ} \mathrm{C}\right)$ dargestellt. Die für die verwendeten pyrolitischen Mantelzusammensetzungen (60\% O1, 25\% Opx und 15\% Cpx, RingwooD 1975) berechneten Leitfähigkeiten sind etwas höher als die des SO2-Modells. Die Abweichungen sind jedoch so gering, dass in Systemen mit Olivin als dominierender Phase die Leitfähigkeiten durch das SO2-Modell genähert werden können.

\subsubsection{Partielle Schmelze}

Partielles Schmelzen setzt ein, sobald die Geotherme die Soliduskurve des entsprechenden Gesteins überschreitet. Unter „,normalen“ Bedingungen im oberen Mantel (kein Wasser), schneiden sich Geotherme und Solidus, sowohl für den kontinentalen als auch ozeanischen oberen Mantel nicht. In Abb. 4.5 sind im Temperatur-Tiefen-Diagramm ozeanische und kontinentale Geotherme und die Soliduskurven von Peridotit (Granat-Lherzolith) für verschiedene Mantelbedingungen dargestellt. Starken Einfluss auf den Solidus haben $\mathrm{H}_{2} \mathrm{O}$ oder $\mathrm{CO}_{2}$. Mit einem Anteil von nur 0.4 Gew. \% senkt Wasser den Solidus im oberen Mantel soweit, dass dieser die Geotherme schneidet und sich partielle Schmelzen (< 1 Vol. \%) bilden (WILSON 1989).

Zur Angabe effektiver Leitfähigkeiten partieller Schmelze bedarf es neben der Leitfähigkeiten der Gesteinsmatrix, vor allem des Volumenanteils der Schmelze nebst deren Leitfähigkeit. Labormessungen an Schmelzen ergaben, in Abhängigkeit der Zusammensetzung der jeweiligen Proben, Leitfähigkeiten zwischen 1-10 S/m (PRESNALL et al. 1972; TYBURCZY und WAFF 1983; SATO und IDA 1984; SATO et al. 1986). SATO et al. (1986) untersuchten unter kontrolliertem Sauerstoffpartialdruck an Gabbro im Temperaturbereich $1000^{\circ}-1400^{\circ} \mathrm{C}$ die elektrischen Leitfähigkeiten partieller Schmelzen. Bei $1200^{\circ} \mathrm{C}$ und einem Schmelzanteil von 17\% erhalten sie eine effektive Leitfähigkeit der Probe von $0.1 \mathrm{~S} / \mathrm{m}$. Extrapolation der zwischen $1300^{\circ}$ und $1400^{\circ} \mathrm{C}$ gemessenen Leitfähigkeiten reiner Schmelzen auf $1200^{\circ} \mathrm{C}$ ergeben für die in der Probe enthaltene Schmelze eine Leitfähigkeit von $3 \mathrm{~S} / \mathrm{m}$.

An Schmelzen aus trockenen Tholeiitbasalten ${ }^{5}$ erhalten TYBURCZY und WAFF (1983) zwischen $1200^{\circ}$ und $1400^{\circ} \mathrm{C}$

$$
\sigma_{m}=10^{5.332} e^{-1.533 e V / k_{B} T}
$$

Aus dieser Beziehung und dem SO2-Modell berechnen LiZARRALDE et al. (1995) für ein Zweiphasensystem die effektive Leitfähigkeit aus dem Gesetz von ARCHIE (SCHMELING 1986)

$$
\sigma=\sigma_{S O 2}+\left(\sigma_{m}-\sigma_{S O 2}\right)\left(c_{m}\right)^{n}
$$

\footnotetext{
${ }^{5}$ Die Ausgangszusammensetzung basaltischer Schmelzen kann vereinfacht als pyrolitisch angenommen werden. Die verschiedenen Bezeichnungen bedeuten unterschiedliche Zusammensetzung und Aufschmelzungsgrade des Pyrolits.
} 


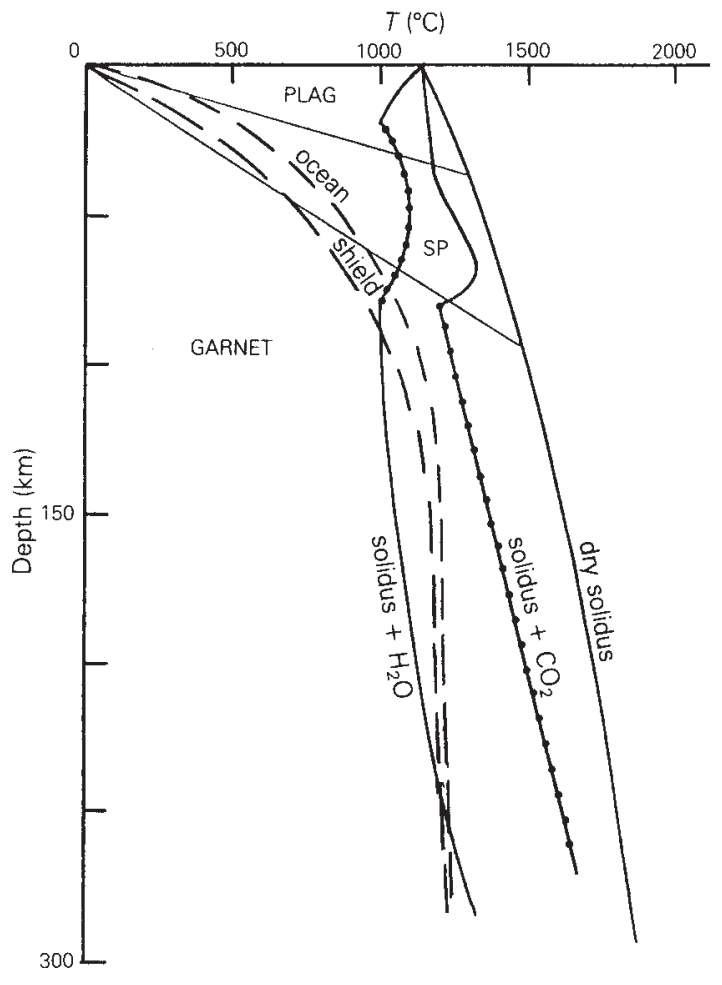

Abbildung 4.5: Soliduskurven für Lherzolith unter trockenen Mantelbedingungen, in Anwesenheit kleiner Mengen $\mathrm{CO}_{2}\left(<5\right.$ Gew. \%) und $\mathrm{H}_{2} \mathrm{O}(<0.4$ Gew. \%). Die gestrichelten Kurven zeigen die ozeanische und kontinentale Mantelgeotherme (nach WYLLIE 1981).

Der Exponent ist mit $n=1.5$ den Bedingungen im oberen Mantel angepasst (Heinson und Constable 1992). Aufgrund der hohen Leitfähigkeit der partiellen Schmelze im Vergleich zu der des Kristalls, erhöhen selbst geringe Mengen Schmelze die effektive Leitfähigkeit signifikant (Abb. 4.6). Ein Schmelzanteil von 3\% in der Probe erhöht deren effektive Leitfähigkeit um ungefähr eine Größenordnung.

Einen anderen Ansatz zur Berechnung der Leitfähigkeit partieller Schmelze wählten RoBERTS und TYBURCZY (1999). In Labormessungen untersuchten sie die effektiven Leitfähigkeiten an partiellen Schmelzen von Fo $_{80}$-Basalt ${ }^{6}$ zwischen $684^{\circ}$ und $1244^{\circ} \mathrm{C}$ bei kontrolliertem Sauerstoffpartialdruck. Mit Hilfe des Programms MELTS (GHIORSO und SACK 1995) berechneten sie für die Probe temperaturabhängig Anteil und chemische Zusammensetzung der Schmelze. Im Vergleich mit den gemessenen effektiven Leitfähigkeiten erhielten sie daraus im betrachteten Temperaturintervall eine konstante Leitfähigkeit der Schmelze von $\sigma_{m}=0.8 \mathrm{~S} / \mathrm{m}$. Die Zunahme der Schmelzleitfä-

${ }^{6}\left(\mathrm{Mg}_{0.80} \mathrm{Fe}_{0.20}\right)_{2} \mathrm{SiO}_{4}$ 


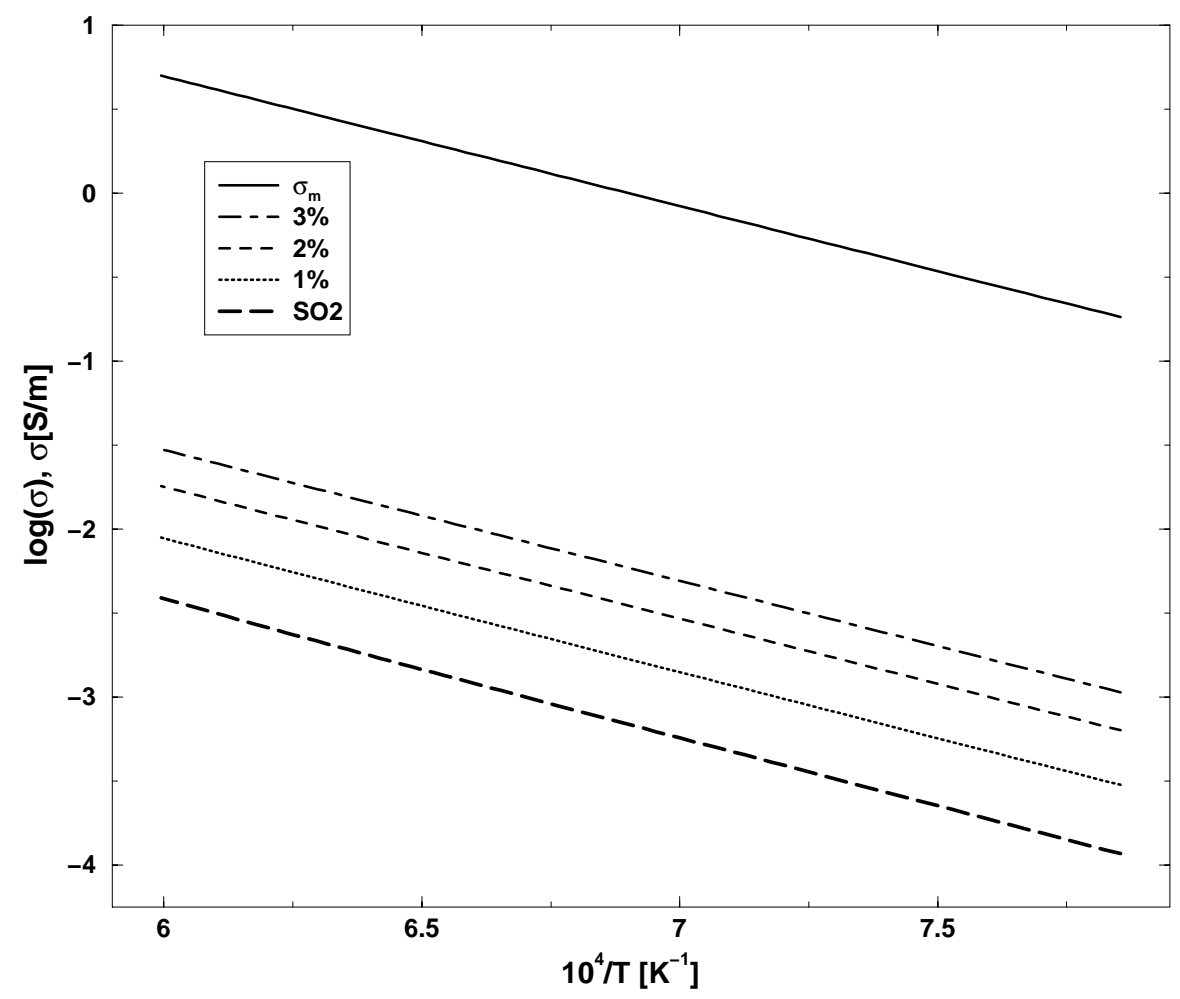

Abbildung 4.6: Leitfähigkeiten partieller Schmelze. Aus den Leitfähigkeitswerten für Schmelze $\sigma_{m}$ und trockenem Olivin $\sigma_{S O 2}$ sind die effektive Leitfähigkeit nach dem Mischungsgesetz von ARCHIE (SCHMELING 1986) berechnet.

higkeit mit der Temperatur wird durch die Änderung der chemischen Zusammensetzung gerade kompensiert.

Mit den Leitfähigkeiten von $\mathrm{Fo}_{80}$-Basalt (HIRSCH et al. 1993) und konstantem $\sigma_{m}$ berechneten ROBERTS und TYBURCZY für verschiedene Mischungsgesetze in Abhängigkeit des Schmelzanteils die effektiven Leitfähigkeiten dieses Zweiphasensystems. Im Temperaturbereich $1100^{\circ}-1200^{\circ} \mathrm{C}$ und mit MELTS berechneten Schmelzanteilen von $0-6 \%$ erhielten sie effektive Leitfähigkeiten zwischen $2 \cdot 10^{-3} \mathrm{~S} / \mathrm{m}(500 \Omega \mathrm{m})$ und $3.2 \cdot 10^{-2} \mathrm{~S} / \mathrm{m}(\sim 30 \Omega \mathrm{m})$.

Ausgehend von diesen Ergebnissen kann eine obere Grenze der elektrischen Leitfähigkeit partieller Schmelzen berechnet werden, die nur vom Anteil der gutleitenden Phase abhängt. Für Kristall und Schmelze werden dazu obere Leitfähigkeitsgrenzen angenommen, wie sie maximal in Labormessungen erhalten wurden. Für verschiedene Mischungsgeset$\mathrm{ze}^{7}$ berechnen sich aus den Maximalleitfähigkeiten des Kristalls $\left(\sigma_{c}^{\max }=10^{-2} \mathrm{~S} / \mathrm{m}\right)$ und

\footnotetext{
${ }^{7}$ Zur Erklärung der in zahlreichen elektromagnetischen Messungen gefundenen hochleitfähigen Zone im lithosphärischen Teil des oberen Mantels wurden von PARTZSCH et al. (2000) ähnliche Berechnungen mit einer (zur Beschreibung auch hoher Schmelzanteile) modifizierten Version des Würfelmodells nach
} 


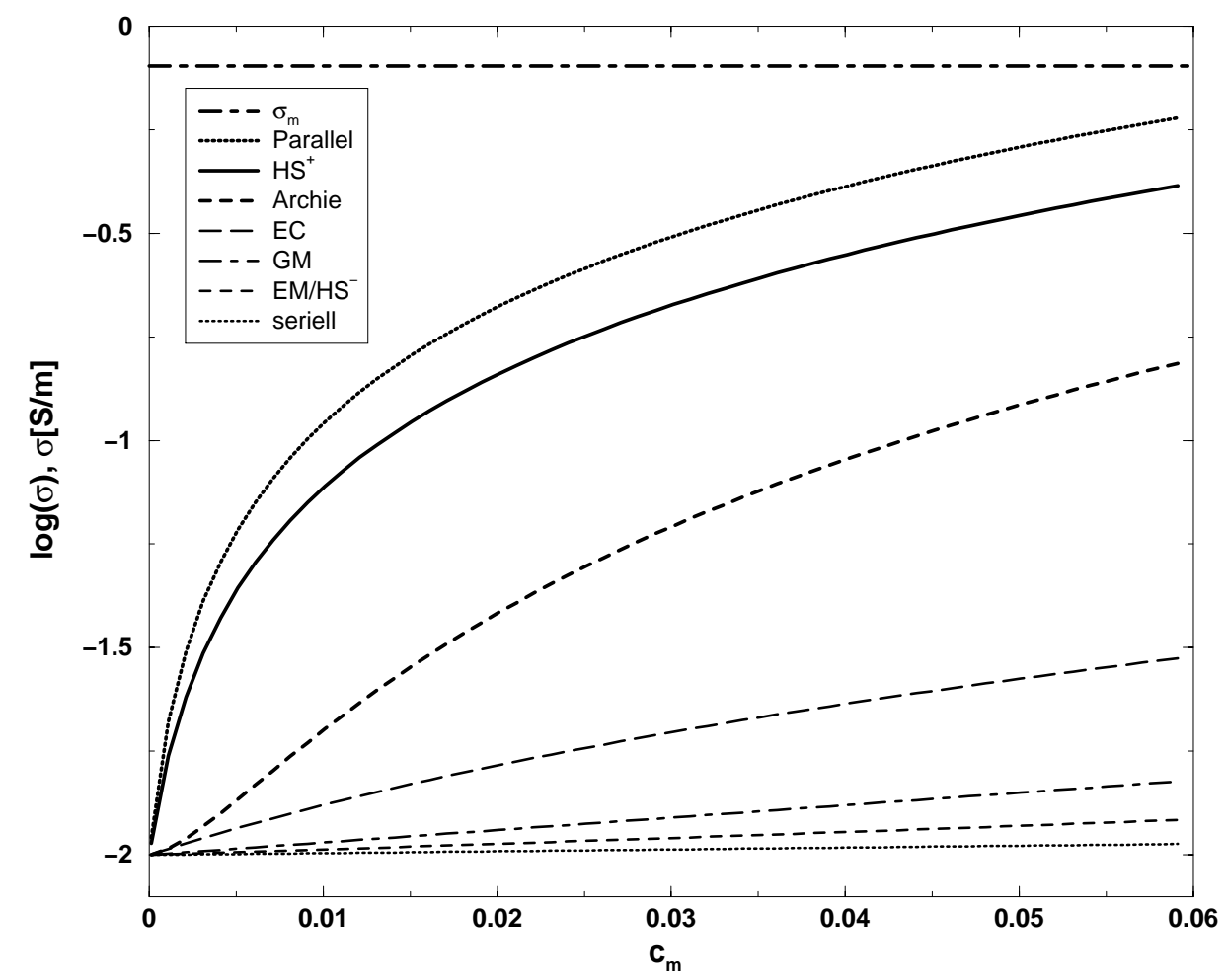

Abbildung 4.7: Maximale Leitfähigkeiten partieller Schmelze in Abhängigkeit des Schmelzanteils. Durch Abschätzung der maximalen Leitfähigkeit von Kristall und Schmelze kann durch Mischungsgesetze frei von Temperaturabhängigkeit oder chemischer Zusammensetzung eine obere Schranke der Leitfähigkeit angegeben werden.

der Schmelze $\left(\sigma_{m}^{\max }=10 \mathrm{~S} / \mathrm{m}\right)$ abhängig vom Schmelzanteil die in Abb. 4.7 dargestellten effektiven Leitfähigkeiten.

Aufgrund des großen Leitfähigkeitskontrastes der beiden Phasen differieren die effektiven Leitfähigkeiten je nach Mischungsgesetz und Konnektivität der gutleitenden Phase um bis zu eineinhalb Größenordnungen. Parallele und serielle Anordnung (A.4 u. A.5) ergeben unabhängig von der Geometrie maximal und minimal mögliche Leitfähigkeiten. Als maximale und minimale effektive Leitfähigkeiten berechnen sich die obere $\mathrm{HS}^{+}$und untere $\mathrm{HS}^{-}{ }^{-}$Grenze. Dabei beschreibt $\mathrm{HS}^{+}$den Zustand eines perfekt verbundenen Netzwerkes der Schmelze. Bei HS ${ }^{-}$liegt sie in isolierten, nicht verbundenen melt pockets vor (z.B. FAUL 1997). Für andere Modellannahmen liegen die effektiven Leitfähigkeiten zwischen $\mathrm{HS}^{+}$und $\mathrm{HS}^{-}$.

WAFF (1974) (Anh. A.6) durchgeführt. 


\subsubsection{Wasser im oberen Mantel}

Die Anwesenheit von Wasser im oberen Mantel hat starken Einfluss auf die physikalischen Eigenschaften des Gesteins. Es ändert die Viskosität und hat damit Einfluss auf die Rheologie von Mantelgestein (KOHLSTEDT et al. 2000), ändert die elastischen Eigenschaften, insbesondere das Schermodul (MeI und KoHLSTEDT 2000a; Mei und Kohlstedt 2000b), senkt den Solidus (vgl. Abb. 4.5) oder führt zu einer Erhöhung der elektrischen Leitfähigkeit.

Die Leitfähigkeit von trockenem Olivin wird dominiert durch die elektronische Leitung durch den Übergang von $\mathrm{Fe}^{2+} \mathrm{zu} \mathrm{Fe}^{3+}$ (SCHOCK et al. 1989). Die Anwesenheit von Wasser im Mantel kann die Leitfähigkeit des Gesteins, durch $\mathrm{H}^{+}$-Ionen als mobilem Ladungsträger zusätzlich erhöhen (KARATO 1990; HIRSCH 1990). Waren im trockenen Olivin die Hauptachsen bezüglich der dort vorherrschenden Leitfähigkeitsmechanismen nur schwach anisotrop (Abb. 4.3), so differieren sie für die Leitfähigkeit durch $\mathrm{H}^{+}$-Ionen um bis zu zwei Größenordnungen. MACKWELL und KOHLSTEDT (1990) bestimmten an Einkristallen von San Carlos Olivin ${ }^{8}$ bei Drucken von bis zu 0.3 GPa im Temperaturbereich $800^{\circ}-1000^{\circ} \mathrm{C}$ die Diffusionskoeffizienten des Wasserstoffs entlang der drei Hauptachsen. Unter der Annahme, die Aktivierungsenthalpie ist entlang aller drei Hauptachsen identisch, erhielten sie

$$
\begin{aligned}
& D_{[100]}^{H^{+}}=(6 \pm 3) \cdot 10^{-5} \cdot e^{-(130 \pm 30 \mathrm{~kJ} / \mathrm{mol}) / R T} \\
& D_{[001]}^{H^{+}}=(5 \pm 4) \cdot 10^{-6} \cdot e^{-(130 \pm 30 \mathrm{~kJ} / \mathrm{mol}) / R T} \\
& D_{[010]}^{H^{+}}=0.01 \cdot D_{[100]}^{H^{+}}
\end{aligned}
$$

wobei sich die Diffusionskoeffizient entlang der Achsen um ungefähr eine Größenordnung unterschieden: $\mathrm{D}_{[100]} \approx 10 \mathrm{D}_{[001]} \approx 100 \mathrm{D}_{[010]}$.

Die meisten Mantelminerale enthalten geringe Mengen Wasser. Diese liegen in Regionen des oberen Mantels bei geringen Konzentrationen nicht als fluide Phase (KoHLSTEDT et al. 1996), sondern gebunden, als $\mathrm{OH}^{-}$- (Bell und RosSMAN 1992) oder $\mathrm{H}^{+}$- Ionen (KARATO 1990; BAI und KOHLSTEDT 1992) vor.

Die von BAI und Kohlstedt (1992) und KohlSTEDT et al. (1996) für $\mathrm{H}^{+}$gemachten Löslichkeitsmessungen wurden von LIZARRALDE et al. (1995) durch

$$
\left[H_{\max }^{+}\right]=10^{-0.61 \pm 0.04} \cdot P^{1.19 \pm 0.01}(\mathrm{MPa})
$$

angepasst. Die Druckabhängigkeit der Löslichkeit von $\mathrm{H}^{+}$-Ionen (hier angegeben in $\mathrm{ppm}^{9} \mathrm{H}^{+} / \mathrm{Si}$ ) ist hierbei vorwiegend durch den Partialdruck von Wasser (und somit auch Sauer-und Wasserstoff) bestimmt (BAI und KoHLSTEDT 1992). Bei diesen Messungen

\footnotetext{
${ }^{8}\left(\mathrm{Mg}_{0.91} \mathrm{Fe}_{0.09}\right)_{2} \mathrm{SiO}_{4}$

${ }^{9} 1 \mathrm{ppm}=10^{-4} \%$
} 


\begin{tabular}{cccc}
\hline $\begin{array}{c}{\left[H_{\max }^{+}\right]} \\
\left(\mathrm{ppm} \mathrm{H} \mathrm{H}^{+} / \mathrm{Si}\right)\end{array}$ & $\begin{array}{c}\text { Druck } \\
(\mathrm{GPa})\end{array}$ & $\begin{array}{c}\text { Temperatur } \\
\left({ }^{\circ} \mathrm{K}\right)\end{array}$ & $\begin{array}{c}\text { Tiefe } \\
(\mathrm{km})\end{array}$ \\
\hline 2225 & 2.1 & 1000 & 70 \\
3525 & 3.1 & 1150 & 100 \\
4920 & 4.1 & 1200 & 130 \\
6226 & 5.0 & 1230 & 157 \\
7995 & 6.1 & 1260 & 192 \\
\hline
\end{tabular}

Tabelle 4.2: $\mathrm{H}^{+}$-Sättigungskonzentrationen in Abhängigkeit von Druck und Temperatur. Zusammenhang zwischen Druck und Temperatur folgen der kontinentalen Geotherme (WYLLIE 1981) und dem PREM-Modell (DZIEWONSKI und ANDERSON 1981).

blieb der Effekt freien Wassers unberücksichtigt und die Sättigungskonzentration ist daher eher als untere Grenze anzusehen (KoHLSTEDT et al. 1996). In Tab. 4.2 sind $\mathrm{H}^{+}$Sättigungskonzentrationen in Abhängigkeit von Druck und Temperatur dargestellt.

Aus den Diffusivitäten (4.7) und der Konzentration der $\mathrm{H}^{+}$-Ionen (4.8) berechnet sich die Leitfähigkeit entlang der Achsen nach der NERnST-EINSTEIN-Gleichung ${ }^{10}$ (4.4)

$$
\sigma_{i}^{H^{+}}=\frac{c_{H^{+}} \cdot D_{i}^{H^{+}} \cdot q^{2}}{k_{B} T}
$$

Abb. 4.8 zeigt die aus (4.9) entlang der [100]- und [010]-Achse berechneten Leitfähigkeiten ${ }^{11}$. Die durchgezogene Kurve stellt die Leitfähigkeit für maximale Sättigungskonzentration an $\mathrm{H}^{+}$-Ionen dar und die gestrichelte die für deren Hälfte. Die Zunahme der elektrischen Leitfähigkeit mit steigender Temperatur ist dabei proportional zur Zunahme der Diffusivität und der Sättigungskonzentration von $\mathrm{H}^{+}$.

Den Berechnungen für kontinentale Geotherme und dem PREM-Modell zufolge entspricht der Temperaturbereich $1150^{\circ}-1265^{\circ} \mathrm{C}$ einer Tiefe von $100-200 \mathrm{~km}$. In diesem Bereich ergeben sich für die [010]-Richtung Leitfähigkeiten zwischen $2 \cdot 10^{-4}$ $3 \cdot 10^{-2} \mathrm{~S} / \mathrm{m}$, entlang der [100]-Achse sind sie um zwei Größenordnungen höher $\left(2 \cdot 10^{-2}<\sigma_{[100]}<3 \mathrm{~S} / \mathrm{m}\right)$. Die Anisotropie der Diffusion entlang der Hauptachsen überträgt sich wegen (4.9) direkt auf die Leitfähigkeit von $\sigma_{[100]} / \sigma_{[010]} \approx 100$. Für die Richtung der [001]-Achse im Olivinkristall liegen die Leitfähigkeiten zwischen den in Abb. 4.8 dargestellten.

\footnotetext{
${ }^{10}$ Für die Berechnung der Leitfähigkeit sind die Einheiten zu beachten. Sind die Konzentrationen von $\left[H_{\max }^{+}\right]$in ppm $\mathrm{H}^{+} / \mathrm{Si}$ angegeben, so ist $\left[\mathrm{c}_{H^{+}}\right]=1 / \mathrm{m}^{3}$.

${ }^{11}$ Für die Darstellung der Leitfähigkeiten in Abb. 4.8 ist die Aktivierungsenthalpie $\Delta \mathrm{H}=130 \mathrm{~kJ} / \mathrm{mol}$. Der Fehler des Vorfaktors ist zur Berechnung der oberen und unteren Grenze jeweils mit berücksichtigt worden. Die Darstellung des Drucks in (4.8) als Funktion der Temperatur erfolgt anhand der kontinentalen Geotherme und des PREM-Modells.
} 


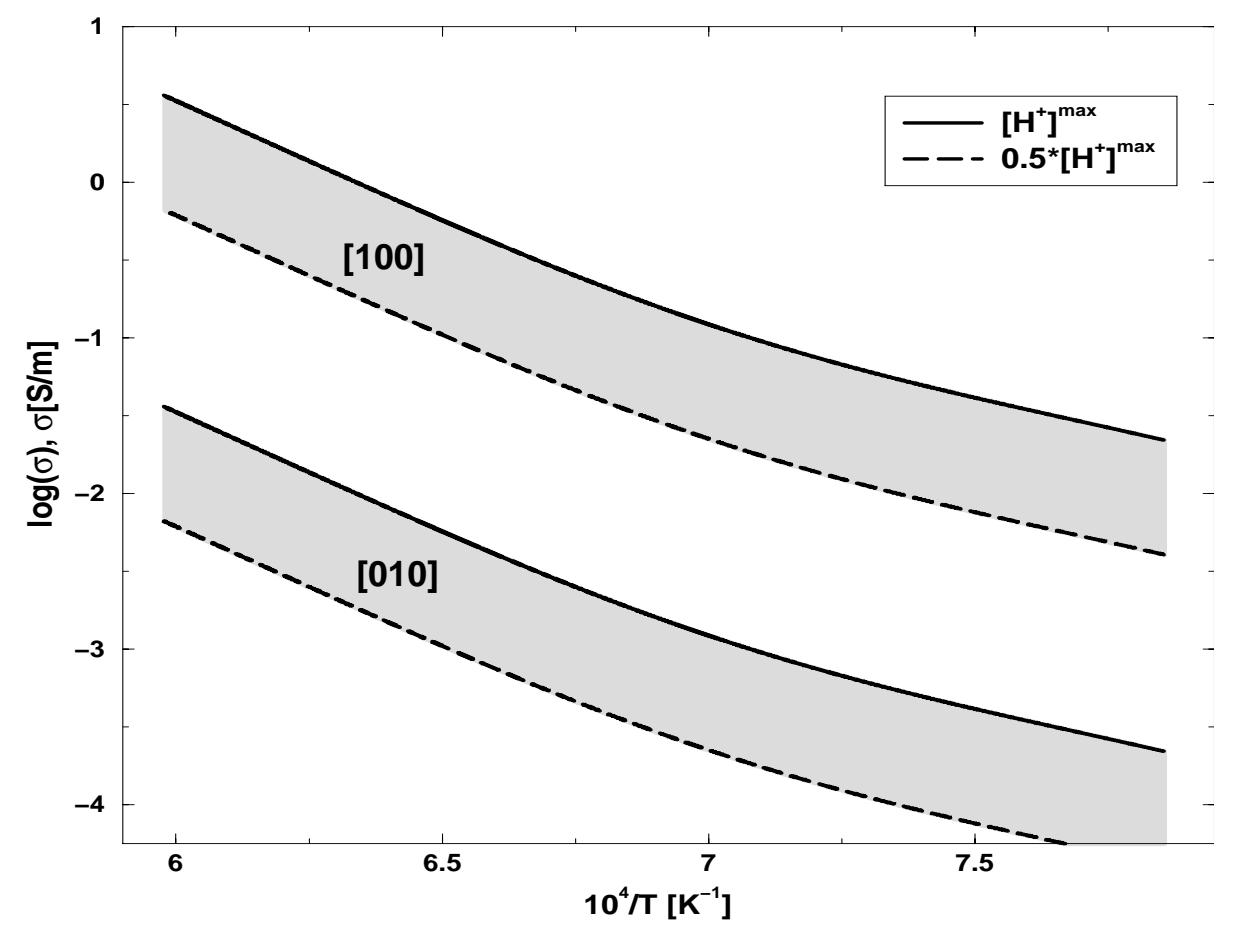

Abbildung 4.8: Leitfähigkeiten durch Wasserstoffdiffusion entlang der [100]- und [010]Achse im Olivinkristall. Die grauen Bereiche geben die aus (4.9) berechneten Leitfähigkeiten zwischen maximaler (obere Kurve) und „halber“ (untere Kurve) Sättigung des Olivin mit $\mathrm{H}^{+}$-Ionen an.

Laboranalysen des Wassergehalts verschiedener magmatischer Gesteine zeigen allgemein weit geringere Konzentrationen $\left(\sim 200-500\right.$ Gew. ppm $\left.{ }^{12}\right)$ als die im Labor bestimmte Sättigungskonzentration (BELL und RosSMAN 1992; JAMTVEIT et al. 2001). Eine mögliche Begründung liegt in der hohen Diffusionsrate von Wasser im oberen Mantel. Schnell aufsteigendes Mantelmaterial bei noch hohen Temperaturen (Xenolithe, $>1000^{\circ} \mathrm{C}$ ) erfährt eine rasche Druckminderung und somit Verringerung des Wasserstoffpartialdruckes. Dies führt, aufgrund der (besonders bei hohen Temperaturen) großen Diffusionskonstanten der $\mathrm{H}^{+}$-Ionen zu einer Dehydrierung der Olivinkristalle während des Aufstiegs (BAI und KoHLSTEDT 1992).

Hochdruckmessungen lassen darauf schließen, dass die im oberen Mantel dominierenden Minerale in der Übergangszone zwischen 2.4 und 2.7 Gew. \% Wasser aufnehmen können (KoHLSTEDT et al. 1996). Obwohl nicht davon auszugehen ist, dass die Minerale dort gesättigt sind, ist anzunehmen, dass (durch Aufströme wie z.B. einem Mantelplume) ge-

\footnotetext{
${ }^{12}$ Für den Vergleich zwischen einer Angabe als Gewichtsanteil (Gew. \%, Gew. ppm) und Konzentrationen in Anzahl $\mathrm{H}^{+}$pro $10^{6} \mathrm{Si}$ (ppm H $\mathrm{H}^{+} / \mathrm{Si}$ ) sind die Dichte sowie genaue Zusammensetzung des Gesteins oder Minerals (insbesondere des Anteils Si) als Informationen notwendig. Für San Carlos Olivin entsprechen $5100 \mathrm{H} / 10^{6} \mathrm{Si}$ einem Gewichtsanteil von 0.03 Gew. \% $\mathrm{H}_{2} \mathrm{O}$ (BAI und KohlstedT 1992).
} 
nügend Wasser in den oberen Mantel gelangen kann, um für Olivin die Sättigungsgrenze zu erreichen (J. Tyburczy, pers. Mitt.).

\subsection{Anisotropie der elektrischen Leitfähigkeit}

Aus dem Vergleich zwischen dem Modellergebnis für den oberen Mantel (Kap. 3) und den im vorigen Kapitel diskutierten Laborergebnissen sind Rückschlüsse auf die dort vorherrschenden Leitfähigkeitsmechanismen möglich.

Das ein solcher Vergleich nicht unproblematisch ist, zeigen Interpretationen der aus Seebodenmessungen gewonnen Mantelleitfähigkeiten in 100-200 km Tiefe um $\sim 0.1 \mathrm{~S} / \mathrm{m}$ (Oldenburg 1981; Oldenburg et al. 1984). Diese hohen Leitfähigkeiten wurden von KARATO (1990) durch Wasserstoffdiffusion in isotropem Olivin ohne Anwesenheit partieller Schmelze erklärt. Erneute Auswertung der Daten von OLdEnBuRG unter Berücksichtigung von Verzerrung durch die Küstenlinie liefern um mehr als eine GröBenordnung geringere Leitfähigkeiten (HEINSON und CONSTABLE 1992). Diese sind allein durch das SO2-Modell zu erklären (CONSTABLE 1993).

Ein derart starker Effekt auf die absoluten Werte der Modellleitfähigkeiten in dieser Arbeit ist, aufgrund der Berücksichtigung von Verzerrung (Kap. 1.2.1) sowie dreidimensionaler Modellierungen unter Einschluss großräumiger Krustenstrukturen (Kap. 3.3) nicht zu erwarten. Darüber hinaus wird zusätzlich auch die Anisotropie mit berücksichtigt.

Die wichtigsten Ergebnisse der im vorherigen Kapitel diskutierten Leitfähigkeitsmechanismen sind in Abb. 4.9 gemeinsam dargestellt, in grau die Ergebnisse für Wasserstoffdiffusion ([100]- und [010]-Achse), in grün die für partielle Schmelze (zwischen 1-3\% Schmelze) und in blau die für trockenen Olivin (zwischen maximaler und minimaler Leitfähigkeit im Kristall). Die rot schraffierten Flächen markieren die aus der 3D-Modellierung erhaltenen horizontalen Hauptleitfähigkeiten für den anisotropen Bereich im oberen Mantel $(100-170 \mathrm{~km})$. Die obere gibt mit $\sim 1 \mathrm{~S} / \mathrm{m}$ die Richtung hoher Leitfähigkeit (WO) und die untere mit $\sim 4 \cdot 10^{-3} \mathrm{~S} / \mathrm{m}$ die dazu senkrechte Richtung (NS) an. Die um mehr als zwei Größenordnungen differierenden Leitfähigkeiten ergeben im Modell einen Anisotropiekoeffizienten von $A>10$.

Trockener Olivin scheidet aufgrund der zu geringen Anisotropie sowie Leitfähigkeiten entlang der Hauptachsen als dominierender Mechanismus im Bereich anisotroper Leitfähigkeit aus. Auch unter Berücksichtigung genauerer Gesteinszusammensetzungen (XU et al. 2000b) und somit geringfügig höherer Leitfähigkeiten (Abb. 4.4) können die Leitfähigkeiten in NS-Richtung, nicht jedoch die hohe Anisotropie erklären.

Die Leitfähigkeit von trockenem Olivin erhöht sich signifikant in Anwesenheit geringer Anteile an Schmelze. Mit Schmelzanteilen von 1-3\% passen die effektiven Leitfähigkeiten im einfachen Modell von LIZARRALDE et al. (1995) die Werte in NS-Richtung an 


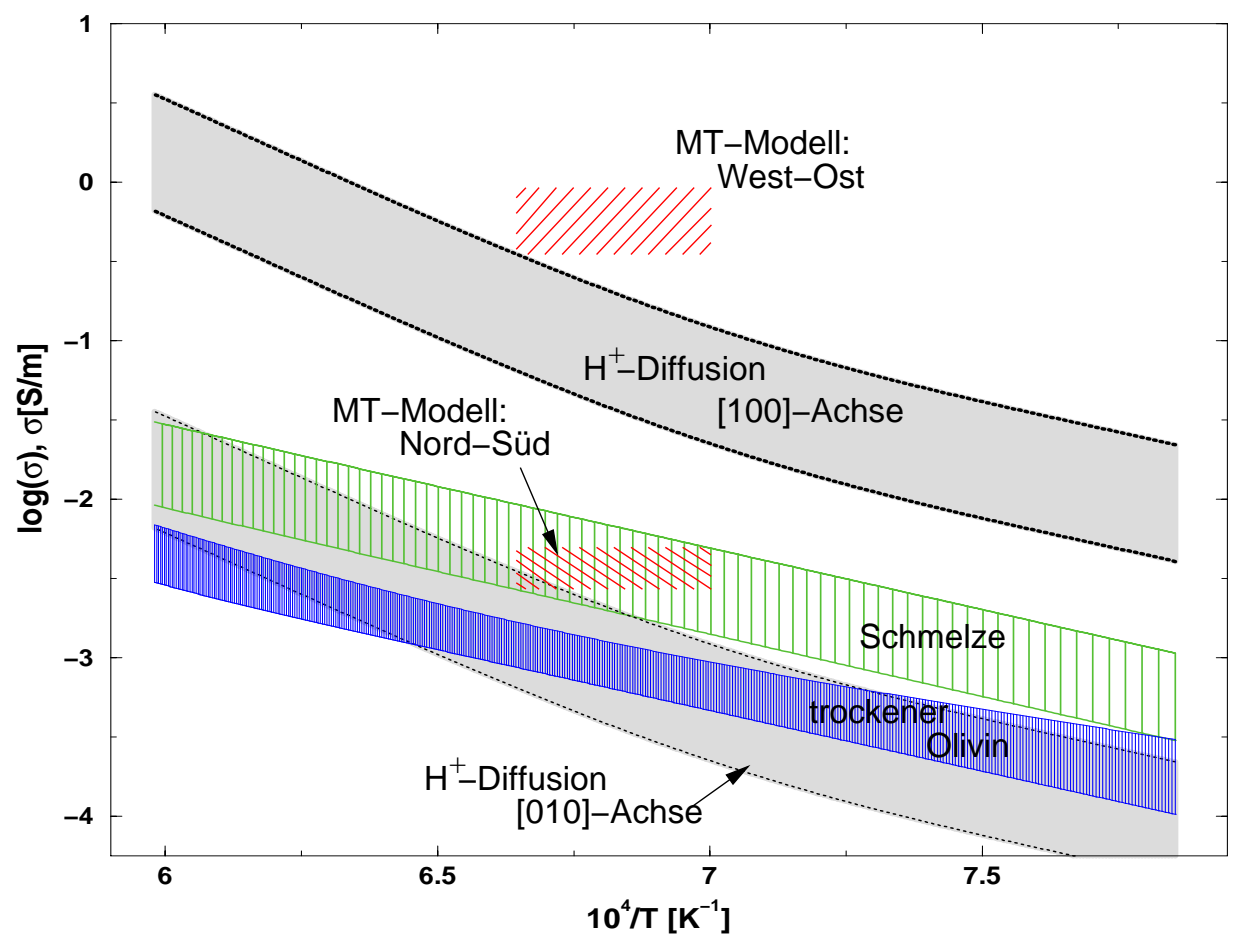

Abbildung 4.9: Vergleich der Modellergebnisse mit den Ergebnissen verschiedener Leitfähigkeitsmechanismen; grau: Wasserstoffdiffusion entlang [100] bzw. [010], grün: partielle Schmelze $(1-3 \%)$, blau: trockener Olivin; rot: Hauptleitfähigkeiten der 3D-Modellierung in $100-170 \mathrm{~km}$ Tiefe, der obere Bereich gibt die Leitfähigkeit in West-Ost-, der untere die in NordSüd-Richtung an.

(Abb. 4.9, grüne Kurve). Zur Erklärung der hohen Leitfähigkeiten senkrecht dazu (WO) sind diese jedoch mehr als eine Größenordnung zu gering.

Die Abschätzungen zur oberen Grenze der effektiven Leitfähigkeiten in Systemen mit partieller Schmelze in Abhängigkeit deren Anteils (Kap. 4.2.2) liefern für $\mathrm{c}_{m}>0.03$ als obere $\left(\mathrm{HS}^{+}\right)$und untere $\left(\mathrm{HS}^{-}\right)$Grenze Leitfähigkeiten in den Größenordnungen der Hauptleitfähigkeiten im Modell. Unter der Annahme richtungsabhängig vernetzter Schmelzen (in einer Richtung sehr gute und senkrecht dazu geringe Konnektivität), können mit hohen Schmelzanteilen Anisotropiekoeffizienten $A>6$ erzeugt werden (Abb. 4.7).

Schmelzanteile von $\mathrm{c}_{m}>0.03$ sind für das Messgebiet in Tiefen zwischen $100-200 \mathrm{~km}$ jedoch deutlich zu hoch. Zur Erklärung der Scherwellengeschwindigkeiten in Teilen des Messgebietes aus dreidimensionaler Scherwellentomographie benötigen KEYSER et al. (2002) Schmelzanteile $<1 \%$. Auch lassen die aus Inversion von Oberflächenwellen bestimmten Geschwindigkeitsmodelle (FRIEDERICH 1998) keine Schmelzanteile $>1 \%$ 


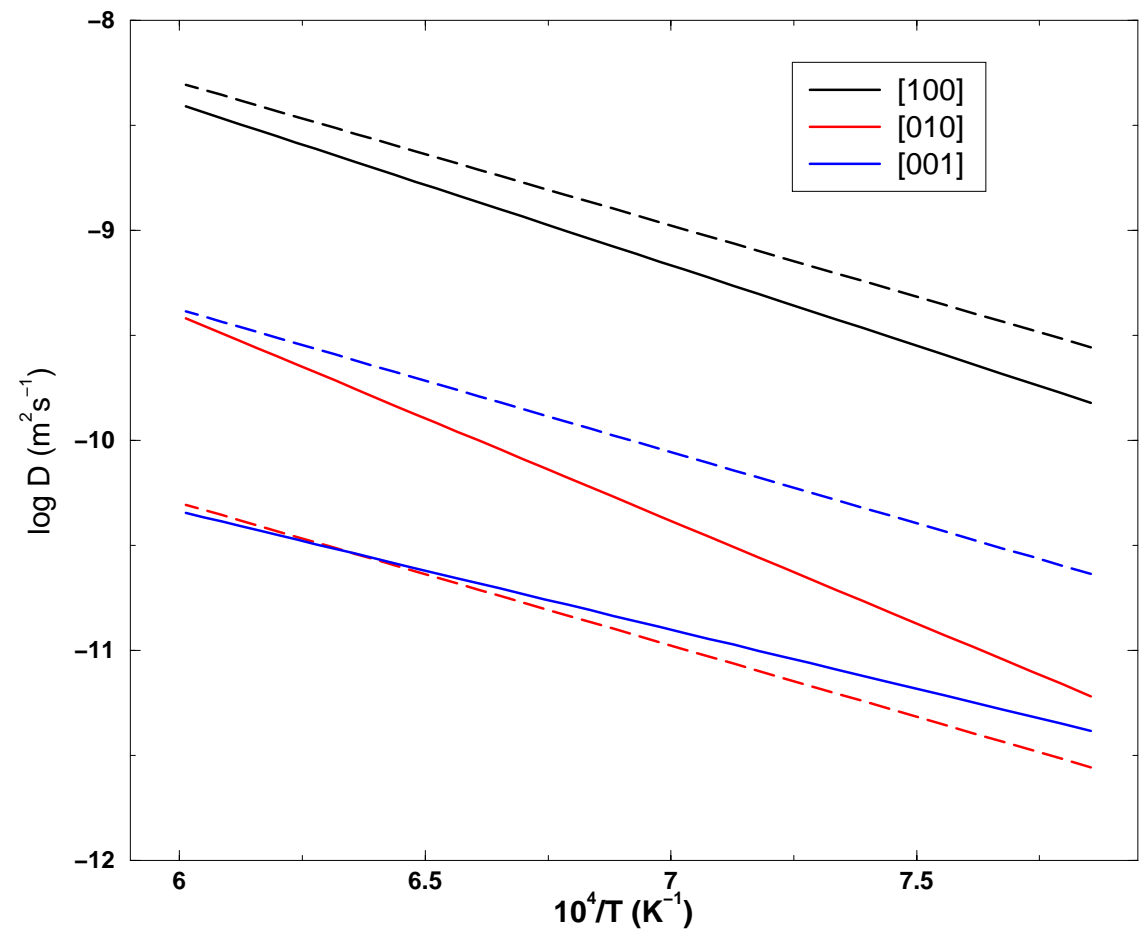

Abbildung 4.10: Diffusionskonstanten für Wasserstoff in Olivin entlang der drei Hauptachsen. Gestrichelt: MACKWELL und KoHLSTEDT (1990); Durchgezogen: KoHLSTEDT und MACKWELL (1998).

zu. Derart geringe Schmelzanteile schließen allerdings partielle Schmelzen als dominierenden Leitfähigkeitsmechanismus aufgrund zu geringer maximaler Anisotropie $(A<3$, Abb. 4.7) und Leitfähigkeiten $\left(\sigma_{H S^{+}}<0.1 \mathrm{~S} / \mathrm{m}\right)$ aus.

Wie in Kapitel 4.2.3 gezeigt, ist die Diffusion von Wasserstoffionen entlang der Hauptachsen im Olivinkristall stark anisotrop. Da Diffusion und Leitfähigkeit einander proportional sind, ist diese Anisotropie direkt auf die Leitfähigkeit übertragbar. Diese in Labormessungen bestimmte, maximale intrinsische Anisotropie $(A \approx 10)$ ist in der Größenordnung der makroskopischen Anisotropie des Leitfähigkeitsmodells $(A>10)$. Dabei werden die im Leitfähigkeitsmodell aufeinander senkrechten Richtungen hoher und niedriger Leitfähigkeit durch die Kristallachsen maximaler und minimaler Diffusivität von $\mathrm{H}^{+}$-Ionen erklärt. Wie der Vergleich in Abb. 4.9 zeigt, stimmen die Größenordnungen der Leitfähigkeiten von [100]-Achse im Kristall und WO-Richtung im Modell, sowie [010] und NS überein. Für beide Richtungen ist allerdings die Kristallleitfähigkeit im Vergleich zum Modell etwas zu gering. Zwei mögliche Erklärungen sind hierfür denkbar. Zum einen muss aufgrund der Vernachlässigung von freiem Wasser bei der Berechnung der Sättigungskonzentration von $\mathrm{H}^{+}$in Olivin diese als untere Grenze angesehen werden (KoHLSTEDT et al. 1996). Eine Erhöhung der Sättigungskonzentration erhöht 
auch die Sättigungs-Leitfähigkeiten entlang der Kristallachsen. Zum anderen schließt der (in Abb. 4.9 nicht dargestellte) Vertrauensbereich von $\sigma_{H^{+}}^{i}$ (durch den Fehler der Aktivierungsenthalpie $\Delta \mathrm{H}$ ) die Leitfähigkeiten des Modells mit ein. Ein geringeres $\Delta \mathrm{H}$ führt auch zu höheren Leitfähigkeiten im Kristall.

In (4.7) ist die Aktivierungsenthalpie der Diffusion von $\mathrm{H}^{+}$in Olivin entlang der Hauptachsen als identisch angenommen. In neueren Labormessungen konnte $\Delta \mathrm{H}$ für die drei Achsen getrennt berechnet werden (KOHLSTEDT und MACKWELL 1998). Wurde die Diffusionskonstante entlang [100] bestätigt, ist sie für die Achse [010] und [001] nach oben bzw. unten korrigiert worden (Abb. 4.10). Die maximale Anisotropie im Kristall bleibt dabei erhalten. War in (4.7) die Richtung geringster Leitfähigkeit die [010]-Achse, ist dies in den neueren Messungen die [001]-Achse. Die bisher getroffenen Aussagen über minimale und maximale Leitfähigkeiten im Olivinkristall durch Wasserstoffdiffusion bleiben erhalten, lediglich die Zuordnung zu den Hauptachsen ist davon betroffen. Für die Diffusionskonstanten folgt aus Abb. 4.10: $D_{[001]} \approx 10 D_{[010]} \approx 100 D_{[001]}$.

Aufgrund der allgemein großen Fehler (besonders der Aktivierungsenthalpie) bei der Berechnung der Diffusionskonstanten sind genauere Messungen erforderlich, um aus der modellierten Leitfähigkeit quantitative Aussagen über die Kristallausrichtung und die Wasserstoffkonzentration machen zu können. Die hohe Anisotropie und die hohe Leitfähigkeit (in WO-Richtung) im Modell bedingen zur Erklärung den Leitfähigkeitsmechanismus entlang der Hauptrichtungen maximaler und minimaler Diffusion im Kristall.

Die Übertragung der intrinsischen elektrischen Anisotropie im Olivinkristall auf makroskopische Anisotropie im gesamten Messgebiet impliziert jedoch neben der Anwesenheit von Wasser im oberen Mantel eine großflächige Ausrichtung der Olivinkristalle. Eine solche Struktur sollte auch Einfluss auf andere geophysikalische Messungen haben. Im Folgenden wird in seismischen Ergebnissen nach weiteren Belegen hierfür gesucht, um diese dann gemeinsam zu interpretieren. 


\section{Gemeinsame Interpretation seismischer und elektrischer Anisotropie}

Deformationen im oberen Mantel sind Ursache für großflächige Anisotropien der seismischen Geschwindigkeiten. Diese beruhen dabei vorwiegend auf der Ausrichtung des Kristallgitters (lattice-preferred orientation, LPO) von Olivin, mit einem Anteil von $70 \%$ das dominierende Mineral im oberen Mantel. Der Zusammenhang zwischen Deformationsregimen und LPO konnte durch numerische Modellierungen und deren Vergleich mit Laborproben erklärt werden (TOMMASI 1998; TOMMASI et al. 1999; Ben Ismail und MAINPRICE 1998). Dabei hat die [100]-Achse von Olivin die Tendenz, sich parallel zur Fließrichtung auszurichten.

Eine verbreitete Methode zur Messung dieser LPO benutzt die Aufspaltung von Scherwellen in anisotropen Medien. Wegen der fehlenden vertikalen Auflösung dieses Scherwellensplittings kann die Tiefenlage, in der die Aufspaltung erfolgt, nicht lokalisiert werden. Zwei Möglichkeiten werden in der Diskussion als wahrscheinlich erachtet. Erfolgt die Aufspaltung im lithosphärischen Teil des oberen Mantels, handelt es sich vermutlich um „fossile“ Anisotropie (frozen flux) (SILVER und CHAN 1991). Diese aus früheren Deformationen erhaltene Anisotropie ist in der kälteren Lithosphere konserviert. Tritt die Anisotropie hingegen in der Asthenosphäre auf, sollte die LPO mit aktuellem Fluss im oberen Mantel verknüpft sein. Dieser kann induziert sein durch Relativbewegung von tektonischen Platten zum oberen Mantel (BARRUOL et al. 1997) oder durch Ströme im oberen Mantel selbst, z.B. durch Mantelplumes (DRICKER et al. 1999; WOLFE et al. 1999).

\subsection{Scherwellensplitting}

\subsubsection{Theorie}

Die Ausbreitung von Scher- oder Transversalwellen in Medien mit anisotroper Ausbreitungsgeschwindigkeit ist in Abb. 5.1 dargestellt. Läuft eine Scherwelle von einem isotropen in ein anisotropes Medium, spaltet sie in zwei zueinander orthogonale 


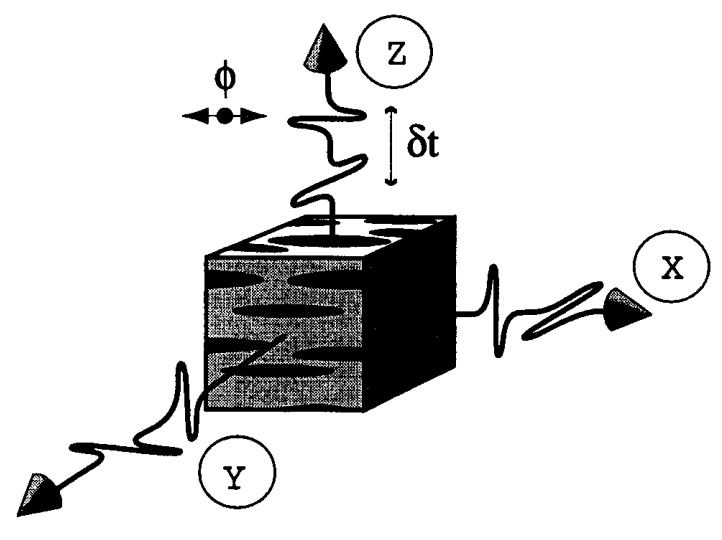

Abbildung 5.1: Bei Ausbreitung von Scherwellen in einem anisotropen Medium spalten diese in zwei senkrecht polarisierte Quasi-Scherwellen unterschiedlicher seismischer Geschwindigkeit auf, hier dargestellt anhand der Ausbreitung in $x-, y-$ und z-Richtung. $\phi$ bezeichnet die schnelle Richtung und $\delta$ t die Zeitdifferenz zwischen beiden (nach SILVER 1996).

Quasi-Scherwellen auf. Die schnellere der beiden ist in Richtung der hohen seismischen Geschwindigkeit $\phi$ polarisiert, die andere senkrecht dazu. Die mit zunehmendem Laufweg in dem anisotropen Medium ansteigende zeitliche Differenz zwischen den Scherwellen wird durch $\delta \mathrm{t}$ angegeben.

Die Beobachtung seismischer Anisotropie erfolgt vorwiegend anhand der Aufspaltung teleseismischer SKS-Phasen. In Abb. 5.2 ist schematisch der Ausbreitungsweg von SKS dargestellt. Von der Quelle breitet sie sich im Mantel zunächst als S-Welle aus, durchquert den äußeren, flüssigen Kern als P-Welle und wird an der Kern-Mantelgrenze auf Empfängerseite wieder in eine S-Welle konvertiert.

Durch die Konversion zur P-Welle im äußeren Kern werden alle anisotropiebedingten Aufspaltungen der Scherwelle auf der Quellseite entfernt. An der empfängerseitigen Kern-Mantelgrenze wird die P-Welle in eine radial, in der Ebene des Großkreises (Ebene in Abb. 5.2) polarisierte S-Welle zurückkonvertiert. Durchläuft die SKS-Phase nach Verlassen des Kerns ein isotropes Medium, wird an der Empfängerstation nur Energie auf der Radialkomponente beobachtet. Ist die Phase jedoch von einem anisotropen Bereich beeinflusst, spaltet die radial polarisierte Scherwelle in zwei orthogonale Quasi-Scherwellen auf. Die Anwesenheit von Energie in transversaler Richtung lässt somit direkt auf einen anisotropen Bereich auf Empfängerseite schließen (SILVER und CHAN 1991). Die laterale Auflösung anisotroper Strukturen ist mit der SKS-Phase sehr gut möglich, da die Strahlen nahezu vertikal im Mantel verlaufen und fast senkrecht an der Erdoberfläche einfallen. Vertikale Aussagen sind jedoch nicht oder nur eingeschränkt möglich, da die 
Quelle der Aufspaltung auf dem gesamten Laufweg zwischen Kern-Mantelgrenze und Erdoberfläche liegen kann. Abschätzungen von SILVER (1996) schränken den Bereich signifikanten Einflusses auf den oberen Mantel ein. Sowohl die Kruste als auch der untere Mantel können hiernach als Quelle von Scherwellensplitting ausgeschlossen werden.

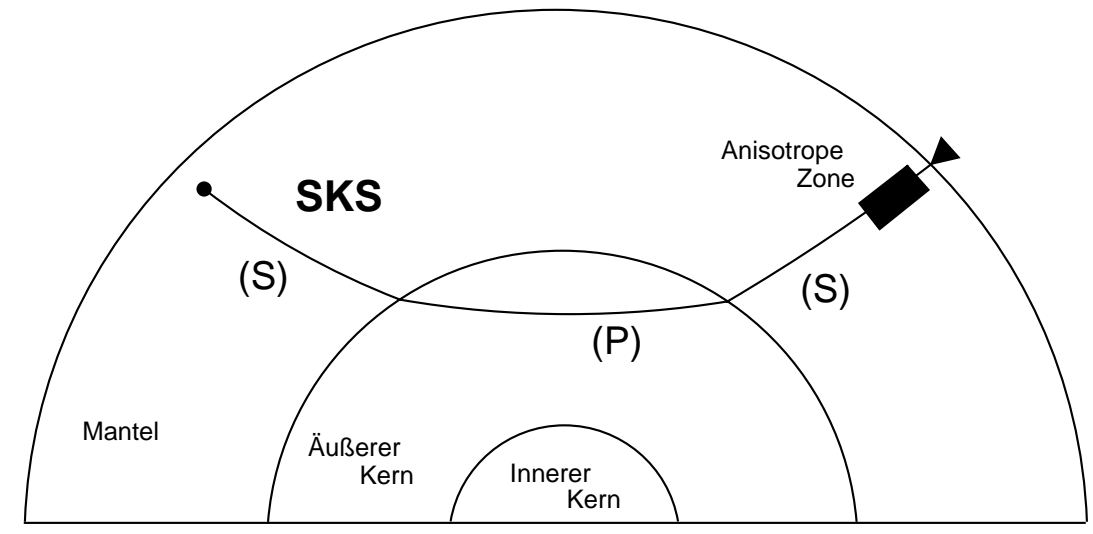

Abbildung 5.2: Schematische Darstellung des Ausbreitungsweges der SKS-Phase in der Erde. Die von der Quelle (Punkt) ausgehende S-Welle durchläuft den äußeren Kern als P-Welle. Bei Konversion von P zu S auf der Empfängerseite entsteht eine radial polarisierte Scherwelle (in der Zeichenebene). Diese fällt am Empfänger (Dreieck) nahezu senkrecht ein.

Das an der Erdoberfläche gemessene Scherwellensplitting wird i.A. durch die Richtung der hohen seismischen Geschwindigkeit $\phi$ und $\delta$, als zeitlicher Differenz zwischen schneller und senkrechter Quasi-Scherwelle, angegeben. Der globale Mittelwert von $\delta \mathrm{t}$ liegt ungefähr bei $1 \mathrm{~s}$ (SILVER 1996).

Für schwache Anisotropie gilt für die zeitliche Verzögerung $\delta \mathrm{t}$

$$
\delta t=L \delta \hat{\beta} / \beta_{0},
$$

mit der Weglänge $L$, der mittleren isotropen Scherwellengeschwindigkeit $\beta_{0}$ und der dimensionslosen mittleren intrinsischen Anisotropie der Scherwellengeschwindigkeit $\delta \hat{\beta}$ (SILVER und CHAN 1991).

\subsubsection{Ergebnisse im Messgebiet}

Auswertungen von Scherwellensplitting-Daten in Mitteleuropa zeigen kein einheitliches Verhalten der Richtung schneller Polarisation. In Abb. 5.3 sind Ergebnisse für Stationen des Deutschen Seismischen Regionalnetzes (German Regional Seismic Network, GRSN) dargestellt (VINNIK et al. 1992; BORMANN et al. 1993; VINNIK et al. 1994). 


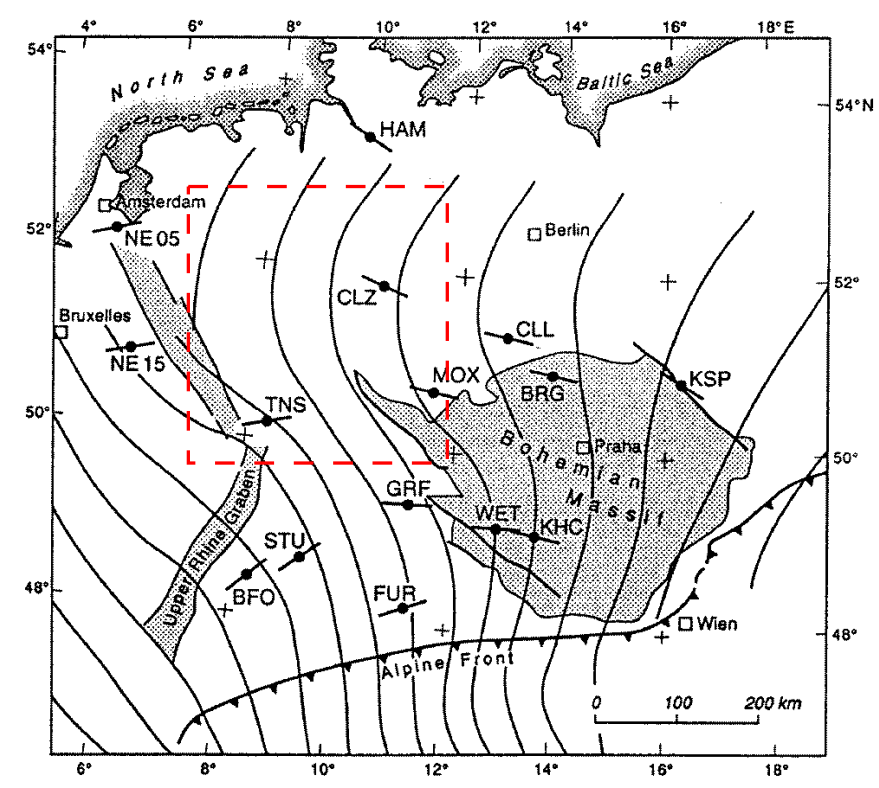

Abbildung 5.3: Scherwellensplitting nördlich der alpinen Front. Dargestellt ist an jeder Station unter der Annahme nur einer anisotropen Schicht die Richtung der Polarisation der schnellen Scherwelle (nach VINNIK et al. 1994). Die rot gestrichelte Kurve umrandet das Gebiet der elektromagnetischen Messungen (vgl. Abb. 2.1).

Für jede Station ist dabei unter Annahme nur einer anisotropen Schicht als Ursache der Aufspaltung, die Richtung der Polarisation der schnellen Scherwelle $\phi$ dargestellt. Diese ergibt sich aus der Mittelung über die Auswertung mehrerer teleseismischer Events unterschiedlichen Backazimuts ${ }^{1} \gamma$.

Trägt man $\phi$ gegen $\gamma$ auf, so wird deutlich, warum eine Mittelung der Richtungen für verschiede Backazimuts nicht sinnvoll ist. In Abhängigkeit von $\gamma$ erhält man an vielen Stationen kein konstantes $\phi$, sondern Werte zwischen $0^{\circ}$ und $90^{\circ}$. In Abb. 5.4 ist dies für die Station GRA1 des Gräfenberg-Arrays (GRF) dargestellt. Die in $\gamma$ erhaltene $90^{\circ}$-Periodizität lässt auf den Einfluss zweier anisotroper Schichten schließen. Die Richtungen $15^{\circ}+k \cdot 90^{\circ}, k=0,1,2,3$ sind dabei transparent windows, Fenster, in denen die einfallende linear polarisierte Welle die untere der beiden anisotropen Schichten nicht wahrnimmt. SKS ist dann in der Ebene polarisiert, die die Richtung der schnellen oder langsamen Quasi-Scherwelle enthält und spaltet somit nicht auf. Da diese Richtungen gerade orthogonal sind, ergibt sich hieraus die $90^{\circ}$-Periodizität. Für diese ist die Aufspaltung der Scherwelle nur von der oberen Schicht beeinflusst, für alle anderen Winkel ergibt sie sich als Überlagerung der Einflüsse beider Schichten.

\footnotetext{
${ }^{1}$ Winkel gegen Nord in die Richtung, aus der die seismische Energie an der Station einfällt.
} 


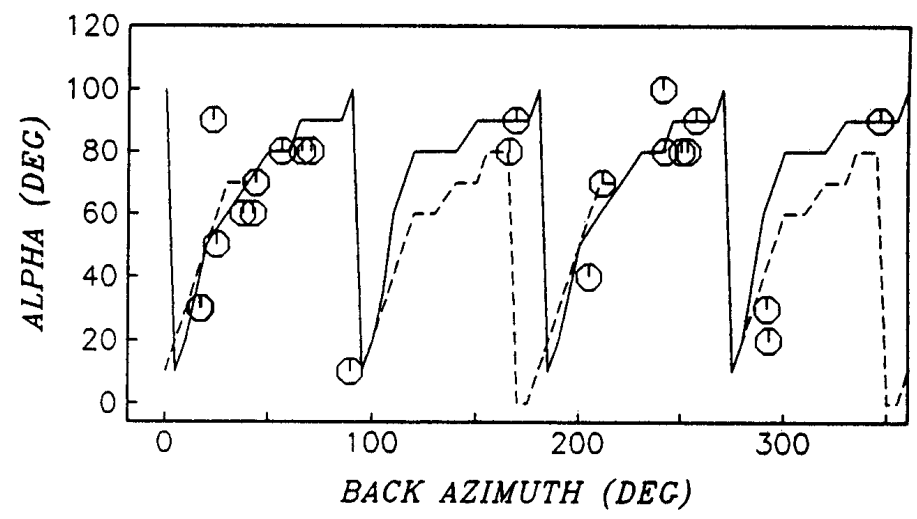

Abbildung 5.4: Abhängigkeit der Richtung $\phi$ vom Backazimut $\gamma$ für die Station GRA1 des Gräfenberg-Arrays. Die Punkte sind die Ergebnisse einzelner Events, die durchgezogene und gestrichelte Kurve sind die Ergebnisse ähnlicher Zwei-Schicht-Modelle (VINNIK et al. 1994).

Für die Stationen BFO, FUR und CLZ ist in Tab. 5.1 das Modell dargestellt, dessen synthetische Seismogramme die Daten der drei Stationen anpassen. Es besteht aus vier Schichten, deren seismische Geschwindigkeiten $\beta_{0}$ linear von Ober- zu Unterkante im angegebenen Intervall zunehmen. Die mittleren der vier Schichten haben horizontal richtungsabhängige Geschwindigkeiten mit intrinsischen Anisotropien $\delta \hat{\beta}$ und die durch $\phi$ angegebenen Richtungen hoher Scherwellengeschwindigkeit. Aus diesen Parametern berechnen sich die zeitliche Differenzen $\delta \mathrm{t}$ (vgl. Gl. 5.1).

\begin{tabular}{cccccc}
\hline Schicht & $\begin{array}{c}\text { Tiefe } \\
(\mathrm{km})\end{array}$ & $\begin{array}{c}\beta_{0} \\
(\mathrm{~km} / \mathrm{s})\end{array}$ & $\delta \hat{\beta}$ & $\begin{array}{c}\phi \\
\left({ }^{\circ}\right)\end{array}$ & $\begin{array}{c}\delta \mathrm{t} \\
(\mathrm{s})\end{array}$ \\
\hline 1 & $0-27$ & $3.2-4.4$ & - & - & - \\
2 & $27-120$ & $4.4-4.5$ & 0.05 & 10 & 1.1 \\
3 & $120-410$ & $4.5-4.7$ & 0.03 & 82 & 1.9 \\
4 & $410-\infty$ & $4.7-5$ & - & - & - \\
\hline
\end{tabular}

Tabelle 5.1: Modellparameter $\phi$ und $\delta$ t des Modells zur Anpassung der Beobachtungen an den Stationen BFO, FUR und CLZ. Von den vier Modellschichten haben nur die mittleren anisotrope Geschwindigkeitsverteilungen (VINNIK et al. 1994).

Aufgrund der Ergebnisse von SILVER (1996) ist eine Unterteilung des Modells in vier Schichten sinnvoll, da für die Kruste (1) und den unteren Mantel (4) keine signifikanten Anisotropien und Aufspaltungen zu erwarten sind. Interpretiert werden die beiden anisotropen Schichten von VINNIK et al. (1994) als lithosphärischer (2) und asthenosphärischer (3) oberer Mantel. Aufgrund der fehlenden vertikalen Auflösung ist das Modell in 
Bezug auf die Schichtmächtigkeiten als eines von vielen möglichen anzusehen. Lediglich die Richtung $\phi$ und $\delta$ sind durch die Daten bestimmt.

Für die obere Schicht ergeben die Auswertungen der SKS-Phasen in Abhängigkeit des Backazimuts im mittleren Abschnitt des Arrays (Abb. 5.3) Winkel zwischen $10^{\circ}$ und $50^{\circ}$, sowie für die schnelle Richtung in der unteren Schicht ungefähr WO $\left(\sim 90^{\circ}\right)$ (VINNIK et al. 1994). Zusätzlich ist in Abb. 5.3 zur räumlichen Einordnung das Gebiet der elektromagnetischen Arraymessungen dargestellt. Dieses überschneidet sich mit dem Bereich seismischer Zwei-Schicht-Anisotropie zwischen CLZ, BFO, FUR und GRF.

\subsection{Interpretation seismischer und elektrischer Anisotropie}

Allein aus der Interpretation der effektiven elektrischen Leitfähigkeit und ihrer Anisotropie ist keine Differenzierung zwischen dem Grad der mittleren Kristallausrichtung von Olivin und der Wasserstoffkonzentration möglich, da beide unabhängig voneinander die Leitfähigkeit beeinflussen. Zur näheren Bestimmung des Verhältnisses können Informationen der im Messgebiet auftretenden seismischen Anisotropie hinzugezogen werden.

Um die elektrische und seismische Anisotropie vergleichen zu können, bedarf es zunächst der Klärung, ob die physikalisch unterschiedlichen Prozesse der richtungsabhängigen Leitfähigkeit und seismischen Geschwindigkeit auf eine identische Ursache zurückzuführen sind.

Für die aus den dreidimensionalen Modellierungen (Kap. 3) erhaltene elektrische Anisotropie im oberen Mantel wurde durch Vergleich mit Labormessungen der zugrundeliegende Mechanismus identifiziert. Dabei konnten die hohe Leitfähigkeit und zugleich hohe Anisotropie in $100-150 \mathrm{~km}$ Tiefe durch (großflächig) ausgerichtete Olivinkristalle in Anwesenheit von Wasser erklärt werden. Für die LPO von Olivin bedeutet dies gerade, dass die [100]-Achse in WO-, die [001] in NS-Richtung und die [010] vertikal ausgerichtet ist.

Der Zusammenhang zwischen elastischen Eigenschaften und LPO von Mantelgesteinen ist gut verstanden. Die Polarisationsrichtung der schnellen Scherwelle $\phi$ (genauso wie die schnelle Ausbreitungsrichtung von Rayleigh- und P-Wellen) verläuft entlang der [100]-Achse im Olivinkristall. Somit kann die Richtung der schnellen Quasi-Scherwelle $\phi$ als Richtung der [100]-Achse interpretiert werden (MAINPRICE und SILVER 1993). Diese liegt für die obere der beiden anisotropen Schichten zwischen $10^{\circ}$ und $50^{\circ}$ und ist für die untere mit $\phi \sim 90^{\circ}$ ungefähr WO ausgerichtet. Da sich im Olivinkristall die seismischen Geschwindigkeiten entlang der zur [100]-Achse senkrechten Hauptachsen nur gering unterscheiden (KUMAZAWA und ANDERSON 1969), ist allein aus 


\begin{tabular}{lcc}
\hline Anisotropie & $\begin{array}{c}\text { Tiefe im } \\
\text { Modell }(\mathrm{km})\end{array}$ & $\begin{array}{c}{[100] \text {-Achse }} \\
\text { im Olivin }\end{array}$ \\
\hline elektrische & $100-150$ & $90^{\circ}$ \\
seismische & $120-410$ & $\sim 90^{\circ}$ \\
\hline
\end{tabular}

Tabelle 5.2: Gegenüberstellung elektrischer und seismischer Anisotropie aus dem 3DLeitfähigkeitsmodell (Kap. 3) und dem Geschwindigkeitsmodell von VINNIK et al. (1994). Die [100]-Achse im Olivin ist die Richtung der Polarisation der schnellen Scherwelle und die hoher elektrischer Leitfähigkeit im angegebenen Tiefenbereich.

Scherwellensplitting-Daten über deren Ausrichtung keine Aussage zu treffen.

Für die untere der beiden Schichten stimmen die Richtung der elektrischen und seismischen Anisotropie - als die Richtung der [100]-Achse im Olivin interpretiert - überein. Die für die Kruste diskutierten Mechanismen, die zu hohen oder richtungsabhängigen Leitfähigkeiten führen, können mit Scherwellensplitting-Daten nicht verglichen werden, da die Kruste aufgrund ihrer geringen Mächtigkeit zu keinen signifikanten Aufspaltungen von Scherwellen führt (SILVER 1996). Auch besteht zwischen der oberen anisotropen Schicht - Schicht (2) im Geschwindigkeitsmodell (Tab. 5.1) - keine Korrelation zu einer anisotropen Schicht im 3D-Leitfähigkeitsmodell (Kap. 3). Es werden daher nur die beiden unteren anisotropen Schichten im Leitfähigkeits- bzw. Geschwindigkeitsmodell betrachtet.

Für diese Schichten kann die Kristallausrichtung von Olivin (LPO) als Ursache der gemeinsamen Anisotropie angenommen werden, da sowohl die Richtung der hohen elektrischen Leitfähigkeit und die Polarisationsebene der schnellen Scherwelle (beide entlang der [100]-Achse) nach WO ausgerichtet sind (Tab. 5.2).

Aufgrund der fehlenden Tiefenauflösung des Scherwellensplittings stimmen in den Modellergebnissen die Tiefenlage der elektrischen und seismischen Anisotropie nicht zwingend überein (s. Tab. 5.2). Dies verhindert zunächst eine gemeinsame Interpretation der Daten, da die aus dem Modell erhaltenen Parameter, die mittlere Anisotropie der Scherwellengeschwindigkeit $\delta \hat{\beta}$ und die Schichtmächtigkeit L, nicht unabhängig voneinander sind (5.1). Ändert sich somit im Modell der Parameter L, so ändert sich zur Anpassung von $\delta t$ auch gleichzeitig die mittlere intrinsische Anisotropie $\delta \hat{\beta}$. Die genaue Kenntnis der Schichtdicke ist daher Voraussetzung zur genauen Angabe der intrinsischen Geschwindigkeitsanisotropie.

Im Folgenden wird ein iteratives Verfahren vorgeschlagen, das durch abwechselnde Modellierung der elektrischen und seismischen Anisotropie die Parameter beider Modelle annähert und dadurch eine gemeinsame Interpretation ermöglicht. In Abb. 5.5 ist diese 


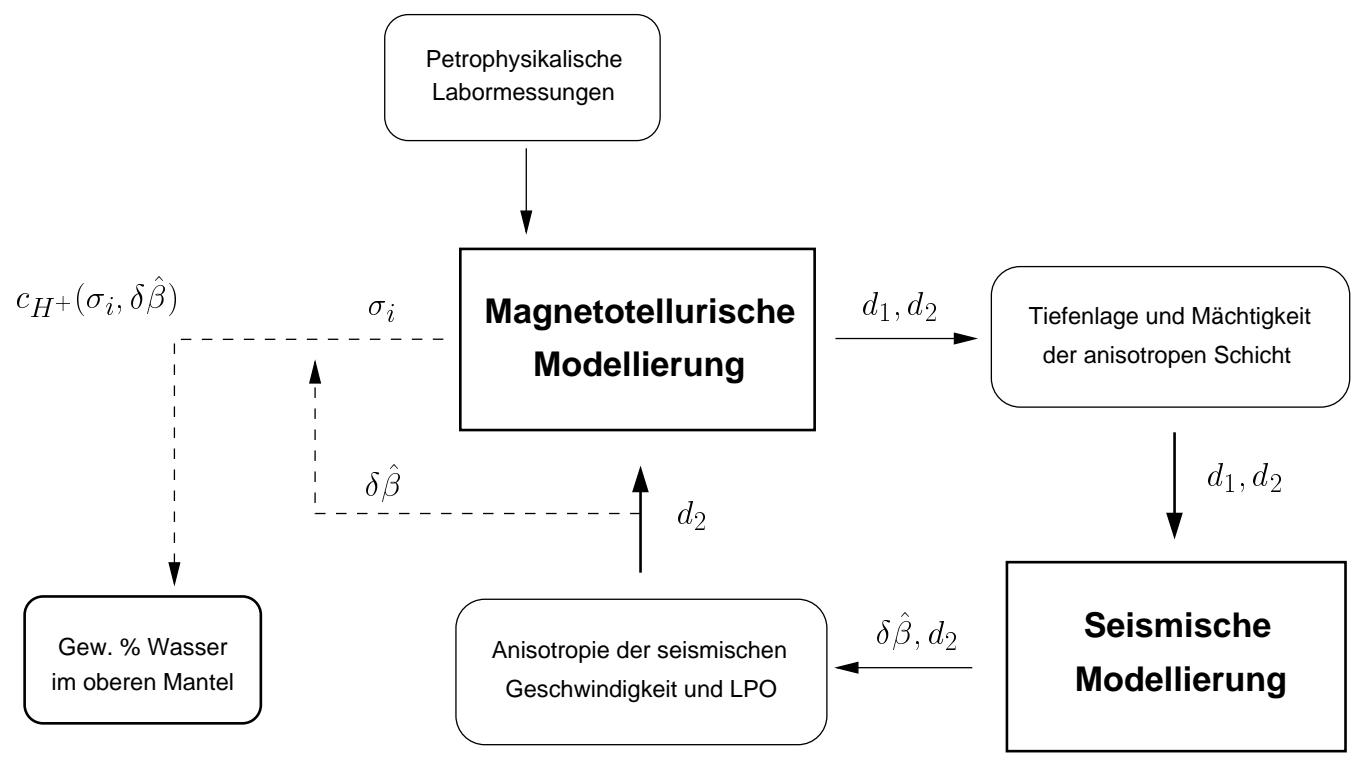

Abbildung 5.5: Gemeinsame Interpretation elektrischer und seismischer Anisotropie durch iterative Anpassung beider Datensätze, mit der Ober- $\left(d_{1}\right)$ und Unterkante $\left(d_{2}\right)$ der anisotropen Schicht, der mittleren Scherwellengeschwindigkeit $\beta_{0}$, mittleren Anisotropie der Scherwellengeschwindigkeit $\delta \hat{\beta}$, den Hauptleitfähigkeiten im Leitfähigkeitsmodell $\sigma_{i}$ und der $\mathrm{H}^{+}-$ Ionen-Konzentration $\mathrm{c}_{H^{+}}$.

Vorgehensweise durch ein Flussdiagramm dargestellt.

Ausgangspunkt ist hierbei die Modellierung der elektrischen Anisotropie, die unter Berücksichtigung petrophysikalischer Ergebnisse der Leitfähigkeiten im oberen Mantel (Kap. 4) die magnetotellurischen Daten (insbesondere die gemeinsam zu erklärende anisotrope Struktur) durch ein minimum structure model anpasst (Kap. 3). Neben der Bestimmung der Streichrichtung der Struktur und den Hauptleitfähigkeiten ist vor allem die Eingrenzung von Schichtdicke und Tiefenlage des anisotropen Leitfähigkeitsbereichs mit Angabe von Oberkante $\left(d_{1}\right)$ und Unterkante $\left(d_{2}\right)$ durch Modellstudien wichtig.

Schichtdicke und Tiefenlage $\left(\mathrm{L}, d_{1}\right)$ werden in der an die magnetotellurische Modellierung anschließenden Modellierung des Scherwellensplittings (seismische Modellierung) als Startparameter übernommen. Durch die Tiefenlage der anisotropen Schicht $\left(d_{1}\right)$ wird ebenfalls die im Modell verwendete tiefenabhängige mittlere isotrope Scherwellengeschwindigkeit $\beta_{0}$ beeinflusst. Für die folgende Anpassung ist die aus der elektromagnetischen Modellierung gut aufgelöste Oberkante $\left(d_{1}\right)$ unveränderlich. Die Unterkante $\left(d_{2}\right)$ und somit die Schichtmächtigkeit L kann dabei - da sich die intrinsische Scherwellenanisotropie nur im Bereich der Ergebnisse von Labormessungen an Gesteinsproben und theoretischen Modellierungen bewegen sollte - variiert werden. Durch Veränderung der Parameter L und $\delta \hat{\beta}$ wird die zeitliche Differenz $\delta t$ (5.1) angepasst. 
Wurde dabei die Tiefenlage der anisotropen Schicht $\left(d_{2}\right)$ modifiziert, wird dies durch Veränderung des Leitfähigkeitsmodells bei der folgenden erneuten magnetotellurischen Modellierung berücksichtigt.

Das Abbruchkriterium dieser abwechselnden Modellierung ist zum einen durch fehlende weitere Verbesserung zwischen zwei Iterationsschritten (optimale gemeinsame Anpassung) oder durch nicht miteinander zu vereinbarende Parameter der Modelle (kein gemeinsames Modell) gegeben.

Im letzteren Fall ist davon auszugehen, dass entgegen der Annahme die Quelle der elektrischen und seismischen Anisotropie in den Daten nicht identisch, oder die zugrundeliegende Struktur komplizierterer Natur ist. Ursache hierfür könnte z.B. ein im Bereich der gemeinsam angepassten Schicht nicht einheitlicher Gehalt an Wasser - und somit von Wasserstoff-Ionen als Ladungsträger - sein.

Sind beide Anisotropien durch ein gemeinsames Modell hinreichend anzupassen, können die Tiefenlage der anisotropen Schicht $\left(d_{1}, L\right)$, deren intrinsische Parameter $\sigma_{i}$ und insbesondere der zuvor schlecht aufzulösende Parameter $\delta \hat{\beta}$ bestimmt werden.

Da letztgenannter Parameter $\delta \hat{\beta}$ ausschließlich von der mittleren Kristallausrichtung im oberen Mantel abhängt, könnte anhand numerischer Berechnungen der LPO Olivins in deformierten Regimen (TOMMASI 1998; TOMMASI et al. 1999; VAUCHEZ et al. 2000) sowie durch Labormessungen erhaltener anisotroper Scherwellengeschwindigkeiten verschiedener Olivingefüge (BEN ISMAIL und MAINPRICE 1998) möglicherweise auf den mittleren Anteil ausgerichteter Olivinkristalle geschlossen werden.

Durch eine so erfolgende Unterscheidung zwischen der auf Kristallausrichtung und der auf Wasserstoffkonzentration beruhenden Leitfähigkeitserhöhung wäre eine genauere Abschätzung des Wassergehaltes im oberen Mantel möglich (vgl. Abb. 5.5). 


\section{Schlussfolgerungen}

Die Interpretation der langperiodischen Daten zeigt im Messgebiet einen ausgedehnten Bereich richtungsabhängiger Leitfähigkeit. Diese kann anhand dreidimensionaler Modellierungen durch eine stark anisotrope Leitfähigkeitsverteilung im asthenosphärischen oberen Mantel zwischen 100 und 150 km erklärt werden. Die Hauptleitfähigkeiten (bzw. Widerstände) im Modell-Mantel haben Werte von $4 \cdot 10^{-3} \mathrm{~S} / \mathrm{m}(250 \Omega \mathrm{m})$ in NS- und $1 \mathrm{~S} / \mathrm{m}(1 \Omega \mathrm{m})$ in WO-Richtung und einen Anisotropiekoeffizienten von $A>10$.

Zur signifikanten Erhöhung der Leitfähigkeit im oberen Mantel gegenüber des trockenen Olivins werden allgemein zwei mögliche Mechanismen diskutiert: partielle Schmelzen und Diffusion von Wasserstoff-Ionen.

Zur Erklärung der hohen und gleichzeitig stark anisotropen Leitfähigkeiten im ModellMantel durch Schmelze benötigt man einen Schmelzanteil $>3 \%$. Zugleich muß in Richtung der hohen Leitfähigkeit perfekte und senkrecht dazu geringe Vernetzung der gutleitfähigen Phase vorliegen. Solch hohe Schmelzanteile sind jedoch in diesen Tiefen unter dem Messgebiet nicht zu erwarten. So benötigen z.B. Keyser et al. (2002) zur Erklärung der Ergebnisse dreidimensionaler Scherwellentomographie in ihrem Geschwindigkeitsmodell Schmelzanteile $<1 \%$ und die Geschwindigkeitsmodelle aus Inversion von Oberflächenwellen (FRIEDERICH 1998) schließen partielle Schmelzen $>1 \%$ aus. Mit derart geringen Schmelzanteilen sind die Leitfähigkeiten partieller Schmelzen selbst bei perfekter Vernetzung zu niedrig.

Die Annahme der Diffusion von Wasserstoff-Ionen im Olivinkristall bietet hingegen die Möglichkeit sowohl die hohe Leitfähigkeit als auch die Anisotropie in integrierter Form zu erklären. Durch die hohe Mobilität von Wasserstoff-Ionen ist trotz geringer Konzentrationen die effektive Leitfähigkeit im Olivinkristall gegenüber trockenen Bedingungen deutlich erhöht. Die Diffusion entlang der Hauptachsen im Olivin ist dabei stark anisotrop (KoHLSTEDT und MACKWELL 1998), $D_{[001]} \approx 10 D_{[010]} \approx 100 D_{[001]}$ und überträgt sich durch die NERNST-EINSTEIN-Gleichung direkt auf die Leitfähigkeit des Olivinkristalls.

Bei ausreichender Konzentration von Wasser im oberen Mantel stimmen die hieraus erhaltenen Leitfähigkeiten und die zugehörige Anisotropie mit den modellierten in etwa überein. Die Richtung hoher Leitfähigkeit im Modell (WO) entspricht dabei der 


\begin{tabular}{rccc}
\hline & $\begin{array}{c}\sigma_{\min } \\
(\mathrm{S} / \mathrm{m})\end{array}$ & $\begin{array}{c}\sigma_{\max } \\
(\mathrm{S} / \mathrm{m})\end{array}$ & $\mathrm{A}$ \\
\hline Trockener Olivin & $0.5 \cdot 10^{-3}$ & $1.7 \cdot 10^{-3}$ & $\sim 1.5$ \\
3\% partielle Schmelze & $0.5 \cdot 10^{-3}$ & $1 \cdot 10^{-2}$ & $\sim 2.8$ \\
Wasserstoffdiffusion & $1.2 \cdot 10^{-3}$ & 0.4 & $\sim 10$ \\
3D-Modell & $4 \cdot 10^{-3}$ & 1 & $>10$ \\
\hline
\end{tabular}

Tabelle 6.1: Zusammenstellung der Leitfähigkeitsgrenzen und Anisotropie der verschiedenen Leitfähigkeitsmechanismen und der Modellparameter im Tiefenbereich $100-150 \mathrm{~km} . \sigma_{\min }$ und $\sigma_{\max }$ stehen für minimale und maximale Leitfähigkeiten, A ist der Anisotropiekoeffizient. Im 3D-Leitfähigkeitsmodell entsprechen $\sigma_{\max }$ und $\sigma_{\min }$ den Hauptleitfähigkeiten.

[100]-Achse im Kristall, die Richtung senkrecht dazu (NS) der [001]-Achse. In Tab. 6.1 sind die aus der Betrachtung der Leitfähigkeitsmechanismen gewonnenen Ergebnisse zusammenfassend dargestellt.

Zur Erklärung der hohen Leitfähigkeit durch Wasserstoffdiffusion werden Konzentrationen benötigt, wie sie für Olivin als druckabhängige Sättigungskonzentration angegeben werden. Ob derart hohe Konzentrationen im oberen Mantel möglich sind, ist noch nicht abschließend geklärt. Eine Konzentration von Wasserstoff mit mehr als 5000 ppm pro Si-Atom entspricht dabei ungefähr einem Anteil größer als 0.03 Gew. \% $\mathrm{H}_{2} \mathrm{O}$ (BAI und KohlstedT 1992). Die seismischen Geschwindigkeitsmodelle für den oberen Mantel unter dem Messgebiet erlauben Wasserkonzentrationen bis zu 0.1 Gew. \% ohne Auftreten partieller Schmelzen (J. Ritter, pers. Mitt.). Damit widersprechen die seismischen Messungen nicht den hier getroffenen Annahmen.

Die Übertragung der intrinischen Anisotropie im Olivinkristall auf makroskopische Anisotropie im gesamten Messgebiet impliziert jedoch zusäzlich eine großflächige und gleichmäßige Ausrichtung der Olivinkristalle im oberen Mantel. Die Achse hoher Leitfähigkeit [100] ist dabei West-Ost ausgerichtet, die Achse geringer Leitfähigkeit [001] senkrecht dazu, in horizontaler Ebene, entsprechend der im Leitfähigkeitsmodell erhaltenen horizontalen Hauptleitfähigkeiten. Die [010]-Achse mit dazwischen liegender Leitfähigkeit ist in vertikaler Richtung ausgerichtet.

Für den Bereich des oberen Mantels ergibt sich direkt aus dem Leitfähigkeitsmodell die Ausrichtung der Olivinkristalle (LPO). Aus dieser können - durch Vergleich mit den Modellergebnissen dreidimensionaler Deformationen auf die Kristallausrichtung von Olivin (TOMMASI 1998) - Aussagen über mögliche Deformationsregime im oberen Mantel getroffen werden (VAUCHEZ et al. 1998; TOMMASI et al. 1999). 
Diese großflächige Ausrichtung der Olivinkristalle im oberen Mantel ist im Messgebiet auch anhand der Aufspaltung von Scherwellen zu beobachten.

Allgemein zeigen die Beobachtungen nördlich der Alpen kein einheitliches Verhalten von SKS-Scherwellen und somit keine einheitliche Polarisationsrichtung der schnellen Scherwelle bei Interpretation durch nur eine anisotrope Schicht. Untersuchungen von VINNIK et al. (1994) liefern, für die Betrachtung der backazimutalen Abhängigkeit des Aufspaltens, für das Messgebiet zwei sich überlagernde, horizontal anisotrope Schichten, wobei für die untere der beiden die Richtung hoher seismischer Geschwindigkeit sehr gut mit der Richtung hoher elektrischer Leitfähigkeit korrelliert. Da sowohl elektrische als auch seismische Anisotropie auf der gleichen LPO von Olivin beruhen, ist die Annahme einer gemeinsamen Ursache im oberen Mantel möglich. Aufgrund der fehlenden Tiefenauflösung des Scherwellensplittings ist das von VINNIK et al. angegebene Geschwindigkeitsmodell allerdings nicht eindeutig.

Die elektrische Leitfähigkeit durch Diffusion von Wasserstoff im Olivinkristall berechnet sich aus der NERNST-EINSTEIN-Gleichung temperaturabhängig aus der Konzentration von Wasserstoff-Ionen und der Diffusionskonstanten entlang der betrachteten Hauptachse. Für großflächig ausgerichtete Olivinkristalle hängt die effektive Leitfähigkeit darüber hinaus zusätzlich von dem Anteil ausgerichteter Kristalle ab.

Allein aus der Interpretation elektromagnetischer Ergebnisse ist eine Unterscheidung zwischen diesem Anteil und der Konzentration von Wasserstoff-Ionen nicht möglich.

Einfache Abschätzungen durch Mischungsgesetze liefern lediglich mittlere Leitfähigkeiten und darüber hinaus keine zusätzlichen Informationen über den Anteil ausgerichteter Kristalle.

Diese könnten durch Verknüpfung elektromagnetischer und seismischer Ergebnisse durch abwechselnde Modellierung der jeweiligen Parameter gewonnen werden. Die iterative Modellanpassung würde eine deutlich verbesserte Auflösung der Modellstruktur gestatten, Voraussetzung dafür ist allerdings, dass die Ursache der elektrischen und seismischen Anisotropie identisch ist. Neben der Mächtigkeit und Tiefenlage der anisotropen Schicht würden vor allem die Hauptleitfähigkeiten und die intrinsische Anisotropie der Scherwellengeschwindigkeit $\delta \hat{\beta}$ bestimmt.

Mit letzterer wäre damit ein Schichtparameter aufgelöst, der nur von der mittleren Kristallausrichtung im oberen Mantel abhängt. Durch Vergleich von $\delta \hat{\beta}$ mit (1) der Anisotropie von Scherwellengeschwindigkeiten aus numerischen Berechnungen der LPO von Olivin in deformierten Regimen (TOMMASI 1998; TOMMASI et al. 1999; VAUCHEZ et al. 2000) und (2) der anisotropen Scherwellengeschwindigkeiten aus Labormessungen an verschiedenen Olivingefügen (BEN ISMAIL und MAINPRICE 1998) sind daraus gegebenenfalls zusätzliche Erkenntnisse über den mittleren Anteil ausgerichteter Olivinkristalle zu gewinnen. 
Durch die genaue Kenntnis des Anteils ausgerichteten Olivins im oberen Mantel wäre dann für die Leitfähigkeiten im Modell zwischen Leitfähigkeitserhöhung durch Kristallausrichtung und Wasserkonzentration zu unterscheiden.

Eine genauere Aussage über den Wassergehalt im oberen Mantel würde somit ermöglicht. 


\section{A Anhang}

\section{A.1 Informationen zu den Messstationen}

\begin{tabular}{|l|l|l|l|}
\hline Station & Registrierzeitraum & Östl. L. $\left(^{\circ}\right)$ & Nördl. B. $\left(^{\circ}\right)$ \\
\hline \hline ALS & $30.07 .99-15.09 .99$ & 9.2 & 50.7 \\
BRI & $29.07 .99-27.09 .99$ & 8.5 & 51.5 \\
BUR & $13.08 .99-13.10 .99$ & 10.095 & 50.314 \\
FUL & $30.07 .99-10.10 .99$ & 9.451 & 50.519 \\
GIE & $30.07 .99-09.10 .99$ & 8.641 & 50.693 \\
GOL & $29.07 .99-15.09 .99$ & 9.45 & 51.9 \\
KLE & $12.08 .99-23.09 .99$ & 8.85 & 51.6 \\
NID & $13.08 .99-22.09 .99$ & 9.072 & 50.442 \\
NIE & $29.07 .99-15.09 .99$ & 9.126 & 51.886 \\
SIL & $29.07 .99-15.09 .99$ & 10.164 & 52.057 \\
SMA & $30.07 .99-15.09 .99$ & 10.4 & 50.7 \\
\hline ARN & $17.05 .00-13.06 .00$ & 8.1 & 51.45 \\
BRI & $17.05 .00-13.06 .00$ & 8.5 & 51.5 \\
HER & $23.05 .00-06.06 .00$ & 7.75 & 51.14 \\
\hline
\end{tabular}

Tabelle A.1: Tabelle der im Rahmen dieser Arbeit vermessenen Stationen.

Tabelle A.2: Tabelle der im Rahmen vorheriger Arbeiten vermessenen Stationen.

\begin{tabular}{|l|l|l|l|}
\hline Station & Östl. L. $\left(^{\circ}\right)$ & Nördl. B. $\left(^{\circ}\right)$ & \\
\hline \hline AHR & 7.13008 & 50.52532 & (LEIBECKER 2000) \\
ALT & 7.53339 & 50.73582 & \\
BAD & 6.80689 & 50.56394 & \\
BKR & 7.87532 & 49.79088 & \\
BOO & 7.01430 & 50.29030 & \\
COC & 7.26308 & 50.13359 & \\
\hline
\end{tabular}




\begin{tabular}{|l|l|l|l|}
\hline DAH & 6.43897 & 50.39814 & \\
DAU & 6.92781 & 50.10211 & \\
DIE & 8.04942 & 50.29019 & \\
FOE & 6.72380 & 49.86679 & \\
GOA & 7.64850 & 50.06166 & \\
HIL & 6.65483 & 50.31136 & \\
HON & 6.80169 & 50.42097 & \\
HUR & 6.34412 & 50.51509 & \\
LAH & 7.73146 & 50.30146 & \\
MAY & 7.41792 & 50.34608 & \\
OSB & 6.77756 & 49.66874 & \\
PRU & 6.38231 & 50.28650 & \\
SEL & 7.80044 & 50.53312 & \\
TRA & 7.07843 & 49.86483 & \\
WAL & 6.81325 & 50.23078 & \\
\hline GAM & 10.578 & 50.337 & (BAHR et al. 2000) \\
KHF & 10.502 & 50.297 & \\
LIW & 10.768 & 50.167 & \\
MEL & 10.232 & 50.426 & \\
OLA & 10.365 & 50.231 & \\
SHA & 10.607 & 50.237 & \\
WAR & 10.386 & 50.378 & \\
\hline BRAM & 9.649 & 51.480 & (SCHNEIDER 1998) \\
MEDE & 8.607 & 51.193 & \\
OHMG & 10.338 & 51.460 & \\
PANO & 8.173 & 51.046 & \\
\hline KLEI & 10.662 & 51.361 & (CYPLIK 1996) \\
KRAJ & 10.525 & 51.441 & \\
OBER & 10.881 & 51.322 & \\
\hline BREU & 9.071 & 51.304 & (BANTIN 1996) \\
HEDD & 8.856 & 51.257 & \\
WILH & 9.287 & 51.358 & \\
\hline ALTE & 9.471 & 51.160 & (PETER 1994) \\
ANRO & 10.330 & 51.280 & \\
FLIN & 10.212 & 51.329 & \\
JUHN & 9.774 & 51.462 & \\
\hline & & & \\
\hline
\end{tabular}




\begin{tabular}{|l|l|l|l|}
\hline LEMB & 9.342 & 51.016 & \\
MOLL & 9.846 & 51.396 & \\
NIEN & 9.658 & 51.358 & \\
THAL & 10.054 & 51.352 & \\
VOLL & 9.597 & 51.253 & \\
\hline LAU & 7.482 & 50.034 & (BAHR 1985) \\
RAB & 8.133 & 50.683 & \\
\hline BGW & 8.781 & 51.014 & (FLUCHE 1983) \\
SET & 9.896 & 50.753 & \\
\hline
\end{tabular}

\section{A.2 Verwendete Programme}

\section{A.2.1 Datenprozessing und Auswertung}

Zum Auslesen der Daten aus dem statischen Speicher des RAP-Systems und deren Weiterverarbeitung in ein binäres Speicherformat wurden die Programme

- ramtra (STEVELING) und

- congoe (STEVELING)

verwendet. Die weitere Verarbeitung der Zeitreihen und Bestimmung der Übertragungsfunktionen erfolgte mit den Programmen

- dezidezi (EISEl, BAHr, SteVeling),

- $\operatorname{select}(\mathrm{GU}, \mathrm{BAHR})$,

- semi (SCHNEIDER 2001),

- analys (BAHR) und

- spetra (EISEL, BAHR).

Die Berechnung der dargestellten Größen aus den Übertragungsfunktionen $\underline{\underline{Z}}$ erfolgte mit den Programmen

- emdisp (LEIBECKER 2000) und

- semi (SCHNEIDER 2001). 


\section{A.2.2 3D Vorwärtsmodellierung: mtd3fwd und d3_to_mt}

Das Hauptprogramm mtd3fwd des benutzten 3D-Finite-Differenzen Codes ist die modifizierte Version von d3mtfwd2 von MACKIE und MADDEN (1997). Es berechnet magnetisches und elektrisches Feld an der Oberfläche eines dreidimensionalen Modells der elektrischen Leitfähigkeit bei Anregung durch ein homogenes elektromagnetisches Feld. Der Code basiert auf dem Algorithmus von MACKIE et al. (1994), der ausgehend von der integralen Form der Maxwellgleichungen durch Finite Differenzen Approximation das Magnetfeld berechnet.

Die Wahl des Grids hat sowohl auf Dauer als auch auf Genauigkeit der Rechnung einen entscheidenden Einfluss. In Abhängigkeit der Leitfähigkeitsverteilung im Modell werden die Gridabstände festgelegt, besonders an hohen Leitfähigkeitskontrasten sollte das Grid feiner definiert sein (MACKIE und BOOKER 1999). Allgemein gilt, dass die Konvergenz der elektrischen Felder abnimmt, je irregulärer das Grid ist.

Die aktuell verwendete Version benutzt als Eingabefile ein 3D-Modell, welches in den Randbereichen das Hintergrundmodell mit enthält. Sind zweidimensionale Strukturen am Rand vorhanden, werden diese am Rand fortgesetzt.

d3_to_mt ist ein Hilfsprogramm zur Berechnung der magnetotellurischen Impedanz, des scheinbaren spezifischen Widerstandes und der Phase aus den mit mtd3fwd für die Erdoberfläche berechneten elektrischen und magnetischen Feldgrößen. Zur einfacheren Handhabung ist dieses Hilfsprogramm um die Ausgabe des am Institut für Geophysik in Göttingen verwendeten Formats der Übertragungsfunktionen erweitert worden (Tab. A.3). Für jeden Gitterpunkt an der Modelloberfläche wird für die in der Modellierung berechneten Frequenzen die magnetotellurische Impedanz berechnet und in einem .mtt-File ausgegeben.

Tabelle A.3: Listing der Erweiterung des Hilfsprogramms d3_to_mt.

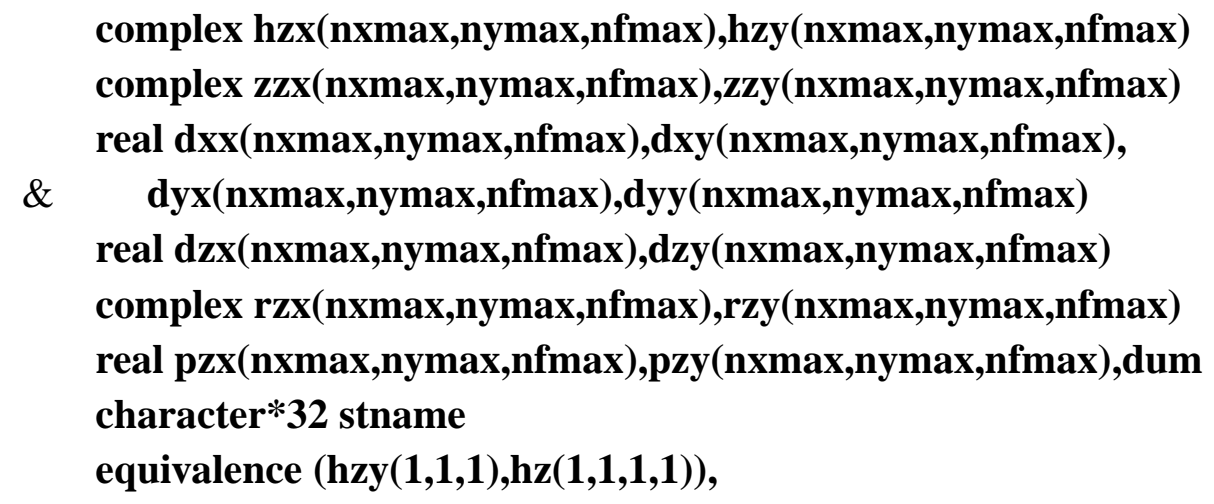


$\&$

$(\operatorname{hzx}(1,1,1), h z(1,1,1,2))$

real alpha2

$\cdots$

alpha2 $=-\mathbf{h x x}(\mathbf{n}, \mathrm{m}, \mathrm{k}) / \mathrm{hxy}(\mathrm{n}, \mathrm{m}, \mathrm{k})$

$\operatorname{zzy}(\mathbf{n}, \mathbf{m}, \mathbf{k})=(\mathbf{h z x}(\mathbf{n}, \mathbf{m}, \mathbf{k})+\operatorname{alpha} 2 * \mathbf{h z y}(\mathbf{n}, \mathbf{m}, \mathbf{k})) /$

\& $\quad(h y x(n, m, k)+a l p h a 2 * h y y(n, m, k))$

$\operatorname{zzx}(\mathbf{n}, \mathbf{m}, \mathbf{k})=\left(\mathbf{h z x}(\mathbf{n}, \mathbf{m}, \mathbf{k})+\operatorname{beta}^{*} \mathbf{h z y}(\mathbf{n}, \mathbf{m}, \mathbf{k})\right) /$

$\&$

$(h x x(n, m, k)+b e t a * h x y(n, m, k))$

...

do $3000 \mathrm{n}=1, \mathrm{nx}$

do $4000 \mathrm{~m}=1$,ny

write(stname,3001) n,m

open $(71$, file=stname,status='new',err=999)

do $5000 \mathrm{k}=1$, nf

c Konjugiert-komplex korrigiert die

c (-iwt)-Abhaengigkeit

$\operatorname{zxx}(\mathbf{n}, \mathbf{m}, \mathbf{k})=\operatorname{conjg}(\operatorname{zxx}(\mathbf{n}, \mathbf{m}, \mathbf{k}) / \mathbf{m u 0} / \mathbf{1 0 0 0})$

$\operatorname{zyx}(n, m, k)=\operatorname{conjg}(\mathbf{z y x}(\mathbf{n}, \mathbf{m}, \mathrm{k}) / \mathbf{m u} 0 / 1000)$

$\operatorname{zxy}(\mathbf{n}, \mathrm{m}, \mathrm{k})=\operatorname{conjg}(\operatorname{zxy}(\mathrm{n}, \mathrm{m}, \mathrm{k}) / \mathrm{mu0} / \mathbf{1 0 0 0})$

$\operatorname{zyy}(n, m, k)=\operatorname{conjg}(z y y(n, m, k) / \mathbf{m u 0} / 1000)$

$\operatorname{zzx}(\mathbf{n}, \mathbf{m}, \mathbf{k})=\operatorname{conjg}(\operatorname{zzx}(\mathbf{n}, \mathbf{m}, \mathbf{k}))$

$\operatorname{zzy}(\mathbf{n}, \mathbf{m}, \mathbf{k})=\operatorname{conjg}(\operatorname{zzy}(\mathbf{n}, \mathbf{m}, \mathbf{k}))$

$\mathbf{z x x i}=\operatorname{imag}(\mathbf{z x x}(\mathbf{n}, \mathbf{m}, \mathbf{k}))$

$\operatorname{zxxr}=\operatorname{real}(\operatorname{zxx}(\mathbf{n}, \mathbf{m}, \mathbf{k}))$

zxyi=imag $(\mathbf{z x y}(\mathbf{n}, \mathbf{m}, \mathbf{k}))$

$\operatorname{zxyr}=\operatorname{real}(\operatorname{zxy}(\mathbf{n}, \mathbf{m}, \mathbf{k}))$

$z y x i=\operatorname{imag}(z y x(n, m, k))$

$\operatorname{zyxr}=\operatorname{real}(\mathbf{z y x}(\mathbf{n}, \mathbf{m}, \mathbf{k}))$

$z y y i=\operatorname{imag}(z y y(n, m, k))$

$\operatorname{zyyr}=\operatorname{real}(\operatorname{zyy}(\mathbf{n}, \mathbf{m}, \mathbf{k}))$

$\operatorname{zzxi}=\operatorname{imag}(\operatorname{zzx}(\mathbf{n}, \mathbf{m}, \mathrm{k}))$

$\operatorname{zzxr}=\operatorname{real}(\operatorname{zzx}(\mathbf{n}, \mathbf{m}, \mathbf{k}))$

zzyi=imag $(z z y(n, m, k))$

$\operatorname{zzyr}=\operatorname{real}(\operatorname{zzy}(\mathbf{n}, \mathbf{m}, \mathbf{k}))$

$\operatorname{dxx}(\mathbf{n}, \mathbf{m}, \mathrm{k})=\operatorname{abs}(\operatorname{zxx}(\mathbf{n}, \mathbf{m}, \mathrm{k})) / \mathbf{1 0 0}$.

$\operatorname{dxy}(n, m, k)=\operatorname{abs}(\operatorname{zxy}(n, m, k)) / 100$. 
$\operatorname{dyx}(n, m, k)=\operatorname{abs}(z y x(n, m, k)) / 100$.

$\operatorname{dyy}(n, m, k)=\operatorname{abs}(z y y(n, m, k)) / 100$.

$\operatorname{dzx}(n, m, k)=\operatorname{abs}(\operatorname{zzx}(n, m, k)) / 100$.

$\operatorname{dzy}(n, m, k)=\operatorname{abs}(\operatorname{zzy}(n, m, k)) / 100$.

write $(71,3002)$ freq $(k)$, dum

write(71,3003) zxxr,zxxi,zxyr,zxyi,zyxr,zyxi,zyyr,zyyi

write $(71,3003) \operatorname{dxx}(n, m, k), d x y(n, m, k), d y x(n, m, k), \operatorname{dyy}(n, m, k)$

$\&$

,zzxr,zzxi,zzyr,zzyi

write(71,3003) dzx(n,m,k),dzy(n,m,k), dum, dum, dum

5000 continue

4000 continue

3000 continue

3001 format('h',i3.3,'v',i3.3,'.mtt')

3002 format (e16.6,i10)

3003 format $(8 F 10.4)$ 


\section{A.3 Mischungsgesetze}

Effektiv-Medium-Theory (EM) (LANDAUER 1952)

$$
\sigma_{E M}=\frac{1}{4}\left[\left(3 c_{1}-1\right) \sigma_{1}+\left(3 c_{2}-1\right) \sigma_{2}+\sqrt{\left(\left(3 c_{1}-1\right) \sigma_{1}+\left(3 c_{2}-1\right) \sigma_{2}\right)^{2}+8 \sigma_{1} \sigma_{2}}\right]
$$

Geometrisches Mittel (GM) (SHANKLAND und DUBA 1990)

$$
\sigma_{G M}=\sigma_{1}^{c_{1}} \cdot \sigma_{2}^{c_{2}}
$$

Effektive Leitfähigkeit (EC) (DEL RIO et al. 1998)

$$
\sigma_{E C}=\sigma_{1} \frac{1+c_{2}\left(\sqrt{\frac{\sigma_{2}}{\sigma_{1}}}-1\right)}{1+c_{2}\left(\sqrt{\frac{\sigma_{1}}{\sigma_{2}}}-1\right)}
$$

Serielle und parallele Lösung (SP) (SCHULGASSER 1976, 1977)

$$
\begin{aligned}
\sigma_{S}^{-1} & =c_{1} / \sigma_{1}+c_{2} / \sigma_{2} \\
\sigma_{P} & =c_{1} \sigma_{1}+c_{2} \sigma_{2}
\end{aligned}
$$

Würfelmodell (WAFF 1974)

$$
\sigma_{e f f} \approx \frac{2}{3} \cdot \beta \cdot \sigma_{1}
$$




\section{Literaturverzeichnis}

BAHR, K. (1985). Magnetotellurische Messungen des elektrischen Widerstandes der Erdkruste und des oberen Mantels in Gebieten mit lokalen und regionalen Leitfähigkeitsanomalien. Doktorarbeit, Universität Göttingen.

BAHR, K. (1988). Interpretation of the magnetotelluric impedance tensor: regional induction and local telluric distortion. J. Geophys., 62:119-127.

BAHR, K. (1991). Geological noise in magnetotelluric data: a classification of distortion types. Phys. Earth Planet. Inter., 66:24-38.

BAHR, K. (1997). Electrical anisotropy and conductivity distribution functions of fractal random networks and of the crust: the scale effect of connectivity. Geophys. J. Int., 130:649-660.

Bahr, K., M. Bantin, C. Jantos, E. Schneider und W. Storz (2000). Electrical anisotropy from electromagnetic array data: implications for the conduction mechanism and for distortion at long periods. Phys. Earth Planet. Inter., 119:237-257.

BAHR, K. und P. BRUTON (1994). Processing of Magnetotelluric Data and their Interpretation. Geomagnetism and Earth's Interior, 4th Training School, Varanasi, 10.10.29.10.94.

BAHR, K. und A. DuBA (2000). Is the asthenosphere electrically anisotropic?. Earth Planet. Sci. Lett., 178:87-95.

BAHR, K. und F. Simpson (2002). Electrical Anisotropy Below Slow-and Fast-Moving Plates: Paleoflow in the Upper Mantle?. Science, 295:1270-1272.

BAI, Q. und D. Kohlstedt (1992). Substantial hydrogen solubility in olivine and implications for water storage in the mantle. Nature, 357:672-674.

BAnTIN, M. (1996). Die elektrische Leitfähigkeit der Erdkruste zwischen Weserbergland und Rheinischem Schiefergebirge - untersucht mit elektromagnetischen Methoden. Diplomarbeit, Institut für Geophysik, Universität Göttingen. 
BARRUOL, G. und W. BEN ISMAIL (2001). Upper mantle anisotropy beneath the African IRIS and Geoscope stations. Geophys. J. Int., 146:549-561.

Barruol, G., G. HelfFrich und A. Vauchez (1997). Shear wave splitting around the northern Atlantic: frozen Pangaean lithospheric anisotropy?. Tectonophysics, 279:135-148.

Bell, D.R. und G. Rossman (1992). Water in Earth's Mantle: The Role of Nominally Anhydrous Minerals. Science, 255:1391-1397.

Ben ISMAIL, W. und D. MAINPRICE (1998). An olivine fabric database: an overview of upper mantle fabrics and seismic anisotropy. Tectonophysics, 296:145-157.

Berdichevsky, M.N. und V. DMitriev (1976). Basic Principles Of Interpretation Of Magnetotelluric Sounding Curves. In: ÁDÁM, A., Hrsg.: Geoelectric and geothermal studies, S. 165-221. Akad. Kiado, Budapest.

Berryman, J.G. (1995). Mixture Theories for Rock Properties. In: Rock Physics and Phase Relations, Bd. 3 d. Reihe A Handbook of Physical Constants, S. 205-228. American Geophysical Union.

Bormann, P., P.-T. Burghardt, L. Makeyeva und L. Vinnik (1993). Teleseismic shear-wave splitting and deformations in Central Europe. Phys. Earth Planet. Inter., 78:157-166.

CAGNIARD, L. (1953). Basic theory of the magneto-telluric method of geophysical prospecting. Geophysics, 18: 605-635.

Chave, A.D. und J. SMith (1994). On electric and magnetic galvanic distortion tensor decompositions. J. Geophys. Res., 99:4669-4682.

CHRISTENSEn, N.I. (1984). The magnitude, symmetry and origin of upper mantle anisotropy based on fabric analysis of ultramafic tectonites. Geophys. J. R. astr. Soc., 76:89-112.

Constable, S. (1993). Conduction by mantle hydrogen. Nature, 362:704.

Constable, S., T. Shankland und A. Duba (1992). The Electrical Conductivity of an Isotropic Olivine Mantle. J. Geophys. Res., 97:3397-3404.

CUll, J.P. (1985). Magnetotelluric soundings over Precambrian contact in Australia. Geophys. J. R. astr. Soc., 80:661-675.

CURrie, C.A., J. CASSIDY und R. Hyndman (2001). A regional study of shear wave splitting above the Cascadia subduction zone: Margin-parallel crustal stress. Geophys. Res. Lett., 28:659-662. 
CyPliK, S. (1996). Magnetotellurik und Erdmagnetische Tiefensondierung an drei Stationen in Nordthüringen. Diplomarbeit, Institut für Geophysik, Universität Göttingen.

De Groot-Hedlin, C. und S. Constable (1990). Occam's inversion to generate smooth, two-dimensional models for magnetotelluric data. Geophysics, 55:16131624.

Debayle, E. und B. Kennett (2000). Anisotropy in the Australasian upper mantle from Love and Rayleigh waveform inversion. Earth Planet. Sci. Lett., 184:339-351.

DeKKeR, D.L. und L. HASTIE (1980). Magneto-telluric impedances of an anisotropic layered earth model. Geophys. J. R. astr. Soc., 61:11-20.

DEL RiO, J.A., R. ZIMMERMAN und R. DAWE (1998). Formula for the conductivity of a two-component material based on the reciprocity theorem. Solid State Commun., 106:183-186.

Dricker, I., L. Vinnik, S. Roecker und L. MAKEYEVA (1999). Upper-mantle flow in Eastern Europe. Geophys. Res. Lett., 26:1219-1222.

Duba, A. und S. Constable (1993). The Electrical Conductivity of Lherzolite. J. Geophys. Res., 98:11885-11899.

Duba, A., H. Heard und R. SchOck (1974). Electrical Conductivity of Olivine at High Pressure and Under Controlled Oxygen Fugacity. J. Geophys. Res., 79:16671673.

DubA, A.G. (1972). Electrical Conductivity of Olivine. J. Geophys. Res., 77:2483-2495.

DubA, A.G. (1976). Are laboratory electrical conductivity data relevant to the Earth?. Acta Geodaet. Geophys. et Montanist. Acad. Sci. Hung., 11:485-495.

DubA, A.G. (1982). Limits to electrical conductivity measurements of silicates. In: SCHREYER, W., Hrsg.: High-Pressure Researches in Geosiences, S. 375-381. E. Schweizerbart'sche Verlagsbuchhandlung, Stuttgart.

Duba, A.G., J. Boland und A. Ringwood (1973). The electrical conductivity of pyroxene. J. Geol., 81:727-735.

Dziewonski, A.M. und D. Anderson (1981). Preliminary Reference Earth Model. Phys. Earth Planet. Inter., 25:297-356.

ECKHARDT, D.H. (1963). Geomagnetic Induction in a Concentrically Stratified Earth. J. Geophys. Res., 68:6273-6278. 
EISEL, M. und V. HAAK (1999). Macro-anisotropy of the electrical conductivity of the crust: a magnetotelluric study of the German Continental Deep Drilling site (KTB). Geophys. J. Int., 136:109-122.

Evans, R.L., P. Tarits, A. Chave, A. White, G. Heinson, J. Filloux, H. Toh, N. Seama, H. Utada, J. Booker und M. Unsworth (1999). Asymmetric Electrical Structure in the Mantle Beneath the East Pacific Rise at $17^{\circ} \mathrm{S}$. Science, 286:752-756.

FAUL, U.H. (1997). Permeability of partially molten upper mantle rocks from experiments and percolation theory. J. Geophys. Res., 102:10299-10311.

FILlOUX, J.H. (1987). Instrumentation and experimental methods for oceanic studies. In: JACOBS, J.A., Hrsg.: Geomagnetism, Bd. 1. Academic Press, London.

FluCHE, B. (1983). Erdmagnetische Tiefensondierung und Magnetotellurik in der Hessischen Senke. Diplomarbeit, Institut für Geophysik, Universität Göttingen.

FRIEDERICH, W. (1998). Wave-theoretical inversion of teleseismic surface waves in a regional network: phase-velocity maps and a three-dimensional upper-mantle shearwave-velocity model for southern Germany. Geophys. J. Int., 132:203-225.

Frost, B.R., W. FyFE, K. TAZAKI und T. CHAN (1989). Grain-boundary graphite in rocks and implications for high electrical conductivity in the lower crust. Nature, 340:134-136.

GAtZemeier, A. (1999). Geoelektrik mit langen Auslagen. Diplomarbeit, Institut für Geophysik, Universität Göttingen.

GATZEMEIER, A. (2000). Langperiodische elektromagnetische Array-Messungen: Anzeichen für einen anisotropen oberen Mantel?. In: HöRDT, A. und J. STOLL, Hrsg.: Prot. 18. Koll. Elektromagnetische Tiefenforschung, Altenberg, S. 148-156.

GHIORSO, M.S. und R. SACK (1995). Chemical mass transfer in magmatic processes, IV, $A$ revised and internally consistent thermodynamic model for the interpolation and extrapolation of liquid-solid equilibria in magmatic systems at elevated temperatures and pressures. Contrib. Mineral. and Petrol., 119:197-212.

Glover, P.W.J. und F. Vine (1994). Electrical conductivity of the continental crust. Geophys. Res. Lett., 21:2357-2360.

Gough, D.I. (1986). Seismic reflectors, conductivity, water and stress in the continental crust. Nature, 323:143-144. 
Groom, R W. und K. BAHR (1992). Corrections for Near Surface Effects: Decomposition of The Magnetotelluric Impedance Tensor and Scaling Corrections for Regional Resistivities: A Tutorial. Surv. Geophys., 13:341-379.

Groom, R.W. und R. BAILEy (1989a). Decomposition of Magnetotelluric Impedance Tensors in the Presence of Local Three-Dimensional Galvanic Distortion. J. Geophys. Res., 94:1913-1925.

Groom, R.W. und R. BAILEy (1989b). Some Effects of Multiple Lateral Inhomogeneities in Magnetotellurics. Geophys. Prosp., 37:697-712.

GROOM, R.W. und R. BAILEY (1991). Analytic investigations of the effects of nearsurface three-dimensional galvanic scatterers on MT tensor decompositions. Geophysics, 56:496-518.

GuÉGuen, Y. und V. Palciauskas (1994). Introduction to the Physics of Rocks. Princeton University Press.

HAAK, V. und R. HUTTON (1986). Electrical resistivity in continental lower crust. In: Dawson, J.B., D. Carswell, J. Hall und K. Wedepohl, Hrsg.: The Nature of the Lower Continental Crust, S. 35-49. Geological Society Special Publication No. 24.

HASHIN, Z. und A. SHTRIKMAN (1962). A variational approach to the theory of effective magnetic permeability of multiphase materials. J. Appl. Phys., 33:3125-3131.

Heinson, G. und S. Constable (1992). The electrical conductivity of the oceanic upper mantle. Geophys. J. Int., 110:159-179.

HIRSCH, L.M. (1990). Enhancing mantle conductivity. Nature, 347:232.

Hirsch, L.M. und T. Shankland (1991a). Determination of Defect Equilibria in Minerals. J. Geophys. Res., 96:377-384.

Hirsch, L.M. und T. Shankland (1991b). Equilibrium Point Defect Concentrations in MgO: Understanding the Mechanisms of Conduction and Diffusion and the Role of Fe Impurities. J. Geophys. Res., 96:385-403.

Hirsch, L.M., T. Shankland und A. Duba (1993). Electrical conduction and polaron mobility in Fe-bearing olivine. Geophys. J. Int., 114:36-44.

Hirth, G., R. Evans und A. Chave (2000). Comparison of continental and oceanic mantle electrical conductivity: Is the Archean lithosphere dry?. Geochem. Geophys. Geosyst., 1:paper number 2000GC000048. 
HöNIG, M. (1998). Magnetotellurik im Rheinischen Schiefergebirge unter Berücksichtigung von Oberflächenverzerrungen und dem Einfluß der anisotropen mittleren Kruste. Diplomarbeit, Institut für Geophysik, Universität Göttingen.

HYNDMAN, R.D. und P. SHEARER (1989). Water in the lower continental crust: modelling magnetotelluric and seismic reflection results. Geophys. J. Int., 98:343-365.

IRIFUNE, T. und A. RINGWOOD (1987). Phase transformations in primitive MORB and pyrolite compositions to $25 \mathrm{GPa}$ and some geophysical implications. In: MANGHNANI, M.H. und Y. SYONO, Hrsg.: High Pressure Research in Mineral Physics, S. 231-242. American Geophysical Union, Washington, DC.

ITO, E. und E. TAKAHASHI (1987). Ultrahigh pressure phase transformations and the constitution of the deep mantle. In: MANGHNANI, M.H. und Y. SYONO, Hrsg.: High Pressure Research in Mineral Physics, S. 221-229. American Geophysical Union, Washington, DC.

Jamtveit, B., R. Brooker, K. Brooks, L. Larsen und T. Pedersen (2001). The water content of olivines from the North Atlantic Volcanic Province. Earth Planet. Sci. Lett., 186:401-415.

Jantos, C. (1998). Audiomagnetotellurische Messungen mit der neuen Göttinger „Midband“-Anlage in Bad Königshofen. Diplomarbeit, Institut für Geophysik, Universität Göttingen.

Ji, S., S. Rondenay, M. Mareschal und G. Senechal (1996). Obliquity between seismic and electrical anisotropies as a potential indicator of movement sense for ductile shear zones in the upper mantle. Geology, 24:1033-1036.

Jödicke, H., J. Untiedt, W. Olgemann, L. Schulte und V. Wagenitz (1983). Electrical Conductivity Structure of the Crust and Upper Mantle Beneath the Rhenish Massif. In: Fuchs, K., K. VON GeHLen, H. MÄLzer, H. MurawsKi und A. Semmel, Hrsg.: Plateau Uplift. The Rhenish Shield - A Case History, S. 288302. Springer-Verlag.

JONES, A.G. (1992). Electrical conductivity of the continental lower crust. In: FounTAIN, D.M., R. ARCUlus und R. KAY, Hrsg.: Continental Lower Crust, S. 81-143. Elsevier, Amsterdam.

JONES, A.G. (1999). Imaging the continental upper mantle using electromagnetic methods. Lithos, 48:57-80.

Junge, A. (1993). Induzierte erdelektrische Felder - neue Beobachtungen in Norddeutschland und im Bramwald. Habil. Math. Nat. Fak., Göttingen. 
KARATO, S. (1990). The role of hydrogen in the electrical conductivity of the upper mantle. Nature, 347:272-273.

Kellett, R.L., M. Mareschal und R. Kurtz (1992). A model of lower crustal electrical anisotropy for the Pontiac Subprovince of the Canadian Shield. Geophys. J. Int., 111:141-150.

KEYSER, M., J. RITTER und M. JORDAN (2002). 3D shear-wave velocity structure of the Eifel plume, Germany. Geophys. J. Int. submitted.

KISSIN, I.G. (1996). Fluid Saturation, Electrical Conductivity, and Seismicity of the Crust. Izvestiya, Physics of the Solid Earth, 32:289-298.

Kohlstedt, D.L., Q. BAI, Z. WANG und S. Mei (2000). Rheology of Partially Molten Rocks. In: Bagdassarov, N., D. LAporte und A. Thompson, Hrsg.: Physics and Chemistry of Partially Molten Rocks, S. 3-28. Kluwer Academic Press.

Kohlstedt, D.L., H. KePPler und D. Rubie (1996). Solubility of water in the $\alpha, \beta$ and $\gamma$ phases of $(\mathrm{Mg}, \mathrm{Fe})_{2} \mathrm{SiO}_{4}$. Contrib. Mineral. and Petrol., 123:345-357.

Kohlstedt, D.L. und S. MACKWELL (1998). Diffusion of Hydrogen and Intrinsic Point Defects in Olivine. Z. Phys. Chem., 207:147-162.

Kumazawa, M. und O. Anderson (1969). Elastic Moduli, Pressure Derivatives, and Temperature Derivatives of Single-Crystal Olivine and Single-Crystal Forsterite. J. Geophys. Res., 74:5961-5972.

Kurtz, R.D., J. Craven, E. Niblett und R. Stevens (1993). The conductivity of the crust and mantle beneath the Kapuskasing Uplift: electrical anisotropy in the upper mantle. Geophys. J. Int., 113:483-498.

LABENDZ, D. (1999). Zwei- und dreidimensionale Widerstandsnetzwerke zur Beschreibung des Leitfähigkeitsmechanismus in der mittleren Kruste. Doktorarbeit, Universität Göttingen.

LANDAUER, R. (1952). The electrical resistance of binary metallic mixtures. J. Appl. Phys., 23:779-784.

Ledo, J., P. QUeRAlT und J. Pous (1998). Effects of galvanic distortion on magnetotelluric data over a three-dimensional regional structure. Geophys. J. Int., 132:295301.

LEIBECKER, J. (2000). Elektromagnetische Arraymessungen im Rheinischen Schiefergebirge: Modelle der elektrischen Leitfähigkeit der Erdkruste und des oberen Mantels mit Verbindungen zum Eifelvulkanismus. Doktorarbeit, Universität Göttingen. 
Leibecker, J., A. Gatzemeier, M. Hönig, O. Kuras und W. Soyer (2002). Evidences of electrical anisotropic structures in the lower crust and the upper mantle beneath Rhenish Shield. Earth Planet. Sci. Lett. revised version submitted.

Lezaeta, P. (2001). Distortion Analysis and 3-D Modeling of Magnetotelluric Data in the Southern Central Andes. Doktorarbeit, Freie Universität Berlin.

Lizarralde, D., A. Chave, G. Hirth und A. Schultz (1995). Northeastern Pacific mantle conductivity profile from long-period magnetotelluric sounding using Hawaii-to-California submarine cable data. J. Geophys. Res., 100:17837-17854.

LOSECKE, W., K. KNÖDEL und W. MÜLLER (1978). Ergebnisse magnetotellurischer Messungen in Norddeutschlan. In: HAAK, V. und J. Homilius, Hrsg.: Prot. Koll. Elektromagnetische Tiefenforschung, Neustadt a. d. Weinstr., S. 41-55.

LOSECKE, W., K. KNÖDEL und W. MüLLER (1979). The conductivity distribution in the North German sedimentary basin derived from widely spaced areal magnetotelluric measurements. Geophys. J. R. astr. Soc., 58:169-179.

MACKIE, R.L. und J. BOOKER (1999). Documentation for mtd3fwd and d3_to_mt. GSYUSA, Inc., 2261 Market St., Suite 643, San Francisco, CA 94114. User Documentation.

MACKIE, R.L. und T. MADDEN (1993). Conjugate direction relaxation solutions for 3-D magnetotelluric modeling. Geophysics, 58:1052-1057.

MACKIE, R.L., T. MAdDEN und P. WANNAMAKER (1993). Three-dimensional magnetotelluric modeling using difference equations - theory and comparisons to integral equation solutions. Geophysics, 58:215-226.

Mackie, R.L., J. SMith und T. MAdDEN (1994). Three-dimensional electromagnetic modeling using finite difference equations: The magnetotelluric example. Radio Science, 29:923-935.

Mackwell, S.J. und D. Kohlstedt (1990). Diffusion of Hydrogen in Olivine: Implications for water in the mantle. J. Geophys. Res., 95:5079-5088.

MAINPRICE, D. und P. SILVER (1993). Interpretation of SKS-waves using samples from the subcontinental lithosphere. Phys. Earth Planet. Inter., 78:257-280.

Mareschal, M., W. Fyfe, J. Percival und T. Chan (1992). Grain-boundary graphite in Kapuskasing gneisses and implications for lower-crustal conductivity. Nature, 357:674-677. 
Mareschal, M., R. Kellett, R. Kurtz, J. Ludden, S. Ji und R. Bailey (1995). Archaean cratonic roots, mantle shear zones and deep electrical anisotropy. Nature, 375:134-137.

Matthes, S. (1996). Mineralogie. Springer-Verlag, Berlin, 5. Aufl.

MAURER, H.-M. (1993). Elektromagnetische Induktion in anisotropen Leitern. Doktorarbeit, Naturwissenschaftliche Fakultät, Universität Braunschweig.

MEI, S. und D. KOHLSTEDT (2000a). Influence of water on plastic deformation of olivine aggregates 1. Diffusion creep regime. J. Geophys. Res., 105:21457-21469.

MEI, S. und D. KOHLSTEDT (2000b). Influence of water on plastic deformation of olivine aggregates 2. Dislocation creep regime. J. Geophys. Res., 105:21471-21481.

Merzer, A.M. und S. Klemperer (1992). High electrical conductivity in a model lower crust with unconnected, conductive, seismically reflective layers. Geophys. J. Int., 108:895-905.

Newman, G.A. und D. Alumbaugh (2000). Three-dimensional magnetotelluric inversion using non-linear conjugate gradients. Geophys. J. Int., 140:410-424.

Nicolas, A. und N. CHRISTENSEN (1987). Formation of anisotropy in upper mantle peridotites - a review. In: FUCHS, K. und C. FroidevauX, Hrsg.: Composition, Structure and Dynamics of the Lithosphere - Asthenosphere System, Bd. 16, S. 111123. Am. Geophys. Union.

Oettinger, G. (1994). Neubearbeitung und Ergänzung eines Magnetotellurik-Profils in der Eifel. In: BAHR, K. und A. Junge, Hrsg.: Prot. 15. Koll. Elektromagnetische Tiefenforschung, Höchst, S. 282-293.

OldenburG, D.W. (1981). Conductivity structure of oceanic upper mantle beneath the Pacific Plate. Geophys. J. R. astr. Soc., 65:359-394.

OldenburG, D.W., K. WhitTAll und R. PARKer (1984). Inversion of Ocean Bottom Magnetotelluric Data Revisited. J. Geophys. Res., 89:1829-1833.

PARTZSCH, G.M., F. SCHILling und J. ARNDT (2000). The influence of partial melting on the electrical behavior of crustal rocks: laboratory examinations, model calculations and geological interpretations. Tectonophysics, 317:189-203.

PETER, C. (1994). Elektrische Leitfähigkeitsmodelle der Erdkruste in Südhannover, Nordhessen und im westlichen Thüringen, abgeleitet aus der elektromagnetischen Induktion durch natürliche Felder. Doktorarbeit, Universität Göttingen. 
Presnall, D.C., C. Simmons und H. Porath (1972). Changes in Electrical Conductivity of a Synthetic Basalt during Melting. J. Geophys. Res., 77:5665-5672.

Ringwood, A.E. (1975). Composition and Petrology of the Earth's Mantle. McGrawHill, New York.

RITTER, P. (1996). Separation of Local and Regional Information in Geomagnetic Response Functions using Hypothetical Event Analysis. Doktorarbeit, University of Edinburgh.

RoBerTS, J.J. und J. TyBURCZY (1991). Frequency dependent electrical properties of polycrystalline olivine compacts. J. Geophys. Res., 96:16205-16222.

Roberts, J.J. und J. TyBURCZY (1993). Frequency dependent electrical properties of dunite as functions of temperature and oxygen fugacity. Phys. Chem. Miner., 19:545-561.

Roberts, J.J. und J. Tyburczy (1999). Partial-melt electrial conductivity: Influence of melt composition. J. Geophys. Res., 104:7055-7065.

RUNCORN, S.K. und D. TOZER (1955). The electrical conductivity of olivine at high temperatures and pressures. Ann. Geophys., 11:98-102.

SATO, H. und Y. IDA (1984). Low frequency electrical impedance of partially molten Gabbro: The effect of melt geometry on electrical properties. Tectonophysics, 107:105-134.

Sato, H., M. Manghnani, B. Lienert und A. Weiner (1986). Effects of sampleelectrode interface polarization on the electrical properties of partially molten rock. J. Geophys. Res., 91:9325-9332.

SaVAGe, M.K. und A. SheEhan (2000). Seismic anisotropy and mantle flow from the Great Basin to the Great Plains, western United States. J. Geophys. Res., 105:13715-13734.

SCHMEling, H. (1986). Numerical models on the influence of partial melt on elastic, anelastic and electrical properties of rocks. Part II: electrical conductivity. Phys. Earth Planet. Int., 43:123-136.

SCHMUCKER, U. (1970). Anomalies Of Geomagnetic Variations In The Southwestern United States, Bd. 13. Bull. Scripps Inst. Oceanography.

SCHMUCKER, U. (1974). Direkte und iterative Verfahren zur Behandlung 2dimensionaler Leitfähigkeitsmodelle. In: BerkTOLD, A., Hrsg.: Prot. Koll. Erdmagnetische Tiefenforschung, Grafrath/Bayern, S. 429-441. 
SCHMUCKER, U. (1987). Substitute Conductors for Electromagnetic Response Estimates. Pure Appl. Geophys., 125:341-367.

SCHNEIDER, E. (1998). Dreidimensionale Modellrechnungen für elektromagnetische Daten eines Profils über die Göttinger D-Anomalie. Diplomarbeit, Institut für Geophysik, Universität Göttingen.

SCHNEIDER, E. (2001). Langperiodische magnetotellurische Messungen auf der oberfächennahen Leitfähigkeitsanomalie in der Münchberger Masse: Hinweise auf eine graphitisierte Überschiebungsfläche durch dreidimensionale Modellrechnungen. Doktorarbeit, Universität Göttingen.

Schock, R.N., A. DUBA und T. SHANKLAND (1989). Electrical Conduction in Olivine. J. Geophys. Res., 94:5829-5839.

SCHULGASSER, K. (1976). Relationship between single-crystal and polycrystal electrical conductivity. J. Appl. Phys., 47:1880-1886.

SCHULGASSER, K. (1977). Bounds on the conductivity of statistically isotropic polycrystals. J. Phys. C: Solid State Phys., 10:407-417.

Shankland, T.J. und A. Duba (1990). Standard electrical conductivity of isotropic, homogeneous olivine in the temperature range $1200^{\circ}-1500^{\circ} \mathrm{C}$. Geophys. J. Int., 103:25-31.

SIEMON, B. (1991). Ein Interpretationsverfahren für induktiv schwach gekoppelte Leitfähigkeitsanomalien, dargestellt am Beispiel des Salzstockes Wesendorf im Gifhorner Trog. Doktorarbeit, Universität Göttingen.

Silver, P.G. (1996). Seismic Anisotropy Beneath the Continents: Probing the Depths of Geology. Annu. Rev. Earth Planet. Sci., 24:385-432.

SILVER, P.G. und W. ChAn (1991). Shear Wave Splitting and Subcontinental Mantle Deformation. J. Geophys. Res., 96:16429-16454.

SIMPSON, F. (1999). Stress and seismicity in the lower continental crust: a challange to simple ductility and implications for electrical conductivity mechanisms. Surv. Geophys., 20:201-227.

SIMPSON, F. (2001a). Fluid trapping at the brittle-ductile transition re-examined. Geofluids, 1:123-136.

SIMPSON, F. (2001b). Resistance to mantle flow inferred from the electromagnetic strike of the Australian upper mantle. Nature, 412:632-635. 
SIMPSON, F. und M. WARNER (1998). Coincident magnetotelluric, P-wave and S-wave images of the deep continental crust beneath the Weardale granite, NE England: seismic layering, low conductance and implications against the fluids paradigm. Geophys. J. Int., 133:419-434.

SMith, J.T. (1997). Estimating galvanic-distortion magnetic fields in magnetotellurics. Geophys. J. Int., 130:65-72.

SMITH, W.H.F. und P. WESSEL (1990). Gridding with continuous curvature splines in tension. Geophysics, 55:293-305.

SOYER, W. (1998). Elektromagnetische Arraymessungen im westlichen Rheinischen Schiefergebirge: Auswertung langer Perioden mit dem Z:H-Verfahren. Diplomarbeit, Institut für Geophysik, Universität Göttingen.

Steveling, E. (1996). Erweiterung des aus Magson-Magnetometer und RAPDatenlogger bestehenden Messystems. In: BAHR, K. und A. Junge, Hrsg.: Prot. 16. Koll. Elektromagnetische Tiefenforschung, Burg Ludwigstein, S. 173-178.

Steveling, E. und M. LeVEn (1994). Stand der RAP-Geräteentwicklung. In: BAHR, K. und A. Junge, Hrsg.: Prot. 15. Koll. Elektromagnetische Tiefenforschung, Höchst, S. 431-438.

SWIFT, C.M. (1967). A magnetotelluric investigation of an electrical conductivity anomaly in the Southwestern United States. Doktorarbeit, MIT, Cambridge, MA.

TING, S.C. und G. HoHMANN (1981). Integral equation modeling of three-dimensional magnetotelluric response. Geophysics, 46:182-197.

TOMmasi, A. (1998). Forward modeling of the development of seismic anisotropy in the upper mantle. Earth Planet. Sci. Lett., 160:1-13.

Tommasi, A., B. TIKOFF und A. VAUCHEZ (1999). Upper mantle tectonics: threedimensional deformation, olivine crystallographic fabrics and seismic properties. Earth Planet. Sci. Lett., 168:173-186.

Tyburczy, J.A. und D. Fisler (1995). Electrical Properties of Minerals and Melts. In: Mineral Physics and Crystallography, Bd. 2 d. Reihe A Handbook of Physical Constants, S. 185-208. American Geophysical Union.

TYBURCZY, J.A. und H. WAFF (1983). Electrical conductivity of molten basalt and andesite to 25 kilobars pressure: Geophysical significance and implications for charge transport and melt structure. J. Geophys. Res., 88:2413-2430. 
VAN'YAn, L.L. und R. HYNDMAn (1996). On the Origin of Electrical Conductivity in the Consolidated Crust. Izvestiya, Physics of the Solid Earth, 32:266-271.

Vauchez, A., A. Tommasi und G. BArruol (1998). Rheological heterogeneity, mechanical anisotropy and tectonics of the continental lithosphere. Tectonophysics, 296:61-86.

Vauchez, A., A. Tommasi, G. Barruol und J. Maumus (2000). Upper Mantle Deformation and Seismic Anisotropy in Continental Rifts. Phys. Chem. Earth (A), 25:111-117.

VinniK, L.P., V. KRishna, R. Kind, P. Bormann und K. StAmmLER (1994). Shear wave splitting in the records of the German Regional Seismic Network. Geophys. Res. Lett., 21:457-460.

Vinnik, L.P., L. Makeyeva, A. Milev und A. Usenko (1992). Global patterns of azimuthal anisotropy and deformations in the continental mantle. Geophys. J. Int., 111:433-447.

Vozoff, K. (1987). The magnetotelluric method. In: NABIGHIAN, M. N., Hrsg.: Electromagnetic Methods in Applied Geophysics - Applications, Bd. 2, S. 641-711. SEG, Tulsa.

WAFf, H.S. (1974). Theoretical Considerations of Electrical Conductivity in a Partially Molten Mantle and Implications for Geothemometry. J. Geophys. Res., 79:40034010.

Wanamaker, B.J. und A. Duba (1993). Electrical Conductivity of San Carlos Olivine Along [100] Under Oxygen- and Pyroxene-Buffered Conditions and Implications for Defect Equilibria. J. Geophys. Res., 98:489-500.

Watson, D.F. (1982). Acord: Automatic contouring of raw data. Comp. \& Geosci., 8:97-101.

WEAVER, J.T. (1990). On the addition of induction vectors. In: HAAK, V. und J. HoMILIUS, Hrsg.: Prot. 13. Koll. Elektromagnetische Tiefenforschung, Hornburg, S. $145-151$.

WEAVER, J.T. (1994). Mathematical Methods for Geo-electromagnetic Induction. Research Studies Press Ltd., New York.

Weidelt, P. (1972). The Inverse Problem of Geomagnetic Induction. Z. Geophysik, 38:257-289. 
Wessel, P. und W. SMITH (1991). Free software helps map and display data. EOS trans. AGU, 72:441-448.

WIESE, H. (1962). Geomagnetische Tiefentellurik II: Die Streichrichtung der Untergrundstrukturen des elektrischen Widerstandes, erschlossen aus geomagnetischen Variationen. Geofis. Pura e appl., 52:83-103.

Wilson, M. (1989). Igneous Petrogenesis. Chapman \& Hall, London.

Wolfe, C.J., F. VERnON III. und A. AL-AMri (1999). Shear-wave splitting across western Saudi Arabia: The pattern of upper mantle anisotropy at a Proterozoic shield. Geophys. Res. Lett., 26:779-782.

Wylegalla, K., G. Bock, J. Gossler, W. Hanka und TOR Working Group (1999). Anisotropy across the Sorgenfrei-Tornquist Zone from shear wave splitting. Tectonophysics, 314:335-350.

WYllie, P.J. (1981). Plate tectonics and magma genesis. Geol. Rundsch., 70:128-153.

XU, Y., C. MCCAmmon und B. Poe (1998a). The effect of alumina on the electrical conductivity of silicate perovskite. Science, 282:922-924.

Xu, Y., B. Poe, T. Shankland und D. Rubie (1998b). Electrical Conductivity of Olivine, Wadsleyite, and Ringwoodite Under Upper-Mantle Conditions. Science, 280:1415-1418.

XU, Y. und T. SHANKLAND (1999). Electrical conductivity of orthopyroxene and its high pressure phases. Geophys. Res. Lett., 26:2645-2648.

XU, Y., T. Shankland und A. Duba (2000a). Pressure effect on electrical conductivity of mantle olivine. Phys. Earth Planet. Inter., 118:149-161.

XU, Y., T. Shankland und B. PoE (2000b). Laboratory-based electrical conductivity in the Earth's mantle. J. Geophys. Res., 105:27865-27875.

YARDLEY, B.W.D. und J. VALLEY (1997). The petrologic case for a dry lower crust. J. Geophys. Res., 102:12173-12185.

Zhamaletdinov, A.A. (1996). Graphite in the Earth's Crust and Electrical Conductivity Anomalies. Izvestiya, Physics of the Solid Earth, 32:272-288.

Zhang, P., L. Pedersen, M. Mareschal und M. Chouteau (1993). Channeling contribution to tipper vectors: a magnetic equivalent to electrical distortion. Geophys. J. Int., 113:693-700. 
Zhdanov, M.S., S. FANG und G. HuRSÁn (2000). Electromagnetic inversion using quasi-linear approximation. Geophysics, 65:1501-1513. 


\section{Index}

Aktivierungsenthalpie, 60, 63, 69, 75

Anisotropie

-koeffizient, 56, 72

elektrisch, 87

makroskopisch, 23, 75, 86

mikroskopisch, intrinsisch, 23, 75, 86

Scherwellen-, 78

seismisch, 77, 84, 87

Asthenosphäre, 11, 56, 76, 80, 85

Auswertefrequenz, 26

Backazimut, 79

Bandlücke, 58

Basalt

$\mathrm{Fo}_{80-}^{-}, 66,67$

Tholeiit-, 65

bivariate Analyse, 26

current channeling, 19

Deformation, 76

Diffusions-

gleichung, 15, 56

koeffizient, 60

konstante, 59, 71, 75

Drehwinkel

phasensensitiv, 20

Swift, 18

Eifel-Projekt, 24

Eindringtiefe, 15, 17, 36, 56

Einkristall, 60

Energieband, 57
Entzerrung, 21, 36

Fluide, 11

frozen flux, 76

Göttinger D-Anomalie, 37, 45

Gabbro, 65

Geotherme, 65

kontinental, 66, 70

ozeanisch, 66

German Regional Seismic Network, 78

Granat, 63

Graphit, 11

Hashin-Shtrikman-Grenze, 61

Hauptleitfähigkeiten, 55, 73, 86

Helmholtzgleichung, 15

Impedanz

modifiziert, 18

Tensor, 15

Induktionspfeile, 22, 37

Klinopyroxen, 57, 63, 65

Konnektivität, 11, 68, 73

Löslichkeit, 69

lattice-preferred orientation, siehe LPO

Leitfähigkeit

anisotrop, 23, 72

effektiv, 60, 65

elektrisch, 14

Halbleiter, 58

integrierte, 56 
Isolator, 58

metallisch, 58

Struktur, regional, 18

Leitungsband, 58

Lherzolith, 66

Granat-, 65

Spinell-, 57

Lithosphäre, 11, 67, 76, 80

LPO, 76, 81, 84

Mantelplume, 71

melt pockets, 68

minimum structure model, 39, 46, 83

Mischungsgesetz, 61

Mobilität, 59

Nernst-Einstein-Gleichung, 60, 70, 85

Norddeutsche Leitfähigkeitsanomalie, 37,44

Ohm'sches Gesetz, 14

Olivin, 57, 63, 65, 76

Hauptachsen, 62, 69, 74

Kristallausrichtung, 75, 86

Leitfähigkeit, 57

San Carlos, 69, 71

trocken, 62, 85

Orthopyroxen, 57, 63, 65

Partialdruck

Sauerstoff-, 61, 62, 65, 66

Wasserstoff-, 71

Peridotit, 57, 65

Perturbationstensor, 22

Phase

Aufspaltung, 29, 34, 54

magnetotellurisch, 16

Polaron, kleines, 63

Polykristall, 60, 62

PREM-Modell, 70

Pyrolit, 65
Rheinisches Schiefergebirge, 24

Scherwellen

-splitting, 76

-tomographie, 85

Quasi-, 77-79

Schiefe phasensensitiv, 20

Swift, 18

Schmelze partiell, 65, 73

Skintiefe, 15, 18

SKS-Phase, 77

SO2-Modell, 62, 72

Solidus, 65, 69

Störstellenleitung, 59

static shift, 19, 40

Streichrichtung, 16

Transformation

$$
\rho^{*}-z^{*}, 17,35,53
$$

transparent window, 79

Triangulation, 30

Übergangszone, 71

Übertragungsfunktion

lokal, 22

normal, 22

Vektor

tellurisch, 19

Verzerrung, 43, 53, 72

frequenzabhängig, 20, 21

frequenzunabhängig, 21

galvanisch, 18, 36

magnetisch, 19

Widerstand

scheinbar spezifisch, 16

Z:H-Verfahren, 21

Zweiphasensystem, 11, 61, 65, 67 


\section{Danksagung}

Allen, die mich bei der Anfertigung dieser Arbeit unterstützt und zu ihrem Gelingen beigetragen haben, möchte ich an dieser Stelle danken.

Mein besonderer Dank gilt hierbei Herrn Prof. Dr. Karsten Bahr, der die Arbeit betreut und ihre Entstehung durch wichtige Hinweise und hilfreiche Unterstützung - selbst über große Entfernungen - begleitet hat. Ihm danke ich weiterhin für die Möglichkeit, meine Arbeit auf zahlreichen internationalen und nationalen Tagungen präsentiert und diskutiert haben zu können.

Herrn Prof. Dr. Andreas Tilgner danke ich für die Übernahme des Korreferats.

Herrn Dr. Jörg Leibecker, Severin Gatzemeier, Denise Tortorella, meinen Eltern und Sabine Flosmann danke ich für die kritische und sehr hilfreiche Durchsicht des Manuskriptes. Besonderer Dank gilt all jenen, die mich während der Messkampagnen unterstützt und mit Rat und Tat zur Seite gestanden haben. Für Hilfe bei technischen Belangen danke ich besonders Herrn Dr. Erich Steveling, Herrn Dr. Martin Leven, Herrn Wilfried Steinhoff, Herrn Jürgen Kruse, Rainer Hennings und Herrn Manfred Herden, die den reibungslosen Ablauf besonders während der Kampagnen garantierten. In diesem Zusammenhang sei für Auf- und Abbau sowie Wartung der Stationen auch Herrn Dr. Jörg Leibecker, Edgar Schneider, Elmar Rothert, Andrea Pfeil, Frank Franken und Severin Gatzemeier gedankt. Dem Institut für Geophysik der Freien Universität Berlin und hier besonders Herrn

Dr. Heinrich Brasse danke ich für das Bereitstellen der Messgeräte und Unterstützung während der Messungen.

Für die gute Zusammenarbeit gilt mein Dank der „Seismologie-Gruppe“, besonders Herrn Dr. Joachim Ritter und Michael Jordan, die mir durch zahlreiche Gespräche und Hinweise weiterhalfen.

Frau Dr. Fiona Simpson danke ich insbesonders für die Möglichkeit, im Rahmen einer Meßkampagne in Australien meinen „Horizont“ zu erweitern.

Der „Kellerbelegschaft“ Dr. Jörg Leibecker und Edgar Schneider danke ich für die vielen schönen Stunden während der Arbeit und darüber hinaus.

Herrn Manfred Herden möchte ich noch einmal gesondert für seine Hilfsbereitschaft bei Problemen aller Art danken.

Frau Schliebe und besonders Frau Eggert-Heise danke ich für die hilfreiche und vor allem geduldige Unterstützung bei Anträgen aller Art.

Abschließend möchte ich Denise danken, die mir während der Erstellung meiner Arbeit hilfreich zur Seite stand und durch viele Diskussionen unterstützte.

$\mathrm{Zu}$ guter Letzt möchte ich mich besonders bei meinen Eltern bedanken, die mich während meines ganzen Studiums und darüberhinaus unterstützt haben. 



\section{Lebenslauf}

\section{Persönliche Daten}

Name:

Geburtsdatum:

Geburtsort:

Staatsangehörigkeit:
Alexander Gatzemeier

9. Mai 1973

Northeim

Deutscher

\section{Schulbildung}

08/1979-06/1983

08/1983-07/1985

08/1985-05/1992

\section{Wehrdienst}

10/1992-09/1993

\section{Studium}

ab 10/1993

10/1995

ab 19/1995

10/1997-04/1999

07/1999

07/1999-01/2002
Grundschule, Rhumspringe

Orientierungsstufe St.-Ursula-Schule, Duderstadt

Eichsfeld-Gymnasium, Duderstadt, Abschluß: Abitur

3./Fernmeldebataillon 1, Hannover

Grundstudium Physik an der Georg-August-Universität Göttingen

Vordiplom Physik

Hauptstudium Physik an der Georg-August-Universität Göttingen, Spezialgebiet Geophysik

Diplomarbeit am Institut für Geophysik der Universität Göttingen in der Arbeitsgruppe von Prof. Dr. Karsten Bahr. Thema der Arbeit: „Geoelektrik mit langen Auslagen“ Diplomprüfung Physik Wissenschaftlicher Mitarbeiter am Institut für Geophysik. Im Rahmen der genannten Finanzierung bestand Gelegenheit zur Promotion.

Thema: „Elektrische Anisotropie durch ausgerichtete Olivinkristalle im oberen Mantel in Mitteleuropa: Magnetotellurische Array-Messungen und ein Ansatz zum Vergleich mit seismischer Anisotropie“ . 